\title{
A review of the carotid artery and facial nerve canal systems in extant turtles
}

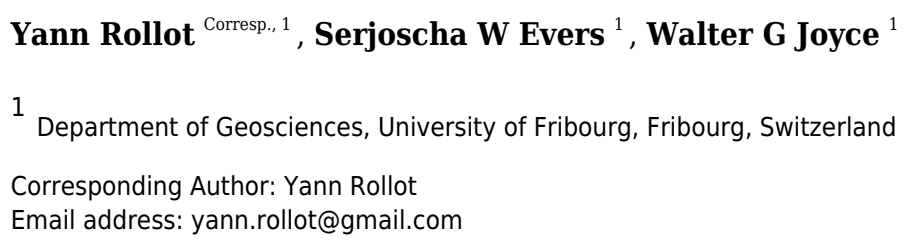

The cranial circulation and innervation systems of turtles have been studied for more than two centuries and extensively used to understand turtle systematics. Although a significant number of studies related to these structures exists, a broader comprehension of variation across the tree has been hindered by poor sampling and a lack of synthetic studies that addressed both systems together. We here provide new insights regarding the carotid circulation and facial nerve innervation systems in a broad set of extant turtles using CT (computed tomography) scans, which allow us to trace the canals these structures form in bone and understand the interaction between both systems. We document that the palatine artery, including the lateral carotid canal, is absent in all pleurodires and carettochelyids and was likely reduced or lost several times independently within Testudinoidea. We also highlight osteological correlates for the location of the mandibular artery. We finally summarize variation regarding the placement of the mandibular artery, location of the geniculate ganglion, placement of the hyomandibular and vidian nerves, and situations where we recommend caution when assessing canals in fossils. A morphometric study confirms that the relative sizes of the carotid canals are correlated with one another. Our results have the potential for building new phylogenetic characters and investigating the circulation systems of fossil taxa, which are expected to shed light on the evolution of the circulation system of turtles and clarify some unresolved relationships between fossil turtle clades. 


\section{A review of the carotid artery and facial nerve canal systems in}

\section{2 extant turtles}

3

4 Yann Rollot ${ }^{1}$, Serjoscha W. Evers ${ }^{1}$, Walter G. Joyce ${ }^{1}$

5

$6{ }^{1}$ Department of Geosciences, University of Fribourg, Fribourg, Switzerland

7

8 Corresponding Author:

9 Yann Rollot

10 Chemin de Musée 6, 1700 Fribourg, Switzerland

11 Email address: yann.rollot@gmail.com 


\section{Abstract}

13 The cranial circulation and innervation systems of turtles have been studied for more than two centuries and extensively used to understand turtle systematics. Although a significant number of studies related to these structures exists, a broader comprehension of variation across the tree has been hindered by poor sampling and a lack of synthetic studies that addressed both systems together. We here provide new insights regarding the carotid circulation and facial nerve innervation systems in a broad set of extant turtles using CT (computed tomography) scans, which allow us to trace the canals these structures form in bone and understand the interaction between both systems. We document that the palatine artery, including the lateral carotid canal, is absent in all pleurodires and carettochelyids and was likely reduced or lost several times independently within Testudinoidea. We also highlight osteological correlates for the location of the mandibular artery. We finally summarize variation regarding the placement of the mandibular artery, location of the geniculate ganglion, placement of the hyomandibular and vidian nerves, and situations where we recommend caution when assessing canals in fossils. A morphometric study confirms that the relative sizes of the carotid canals are correlated with one another. Our results have the potential for building new phylogenetic characters and investigating the circulation systems of fossil taxa, which are expected to shed light on the evolution of the circulation system of turtles and clarify some unresolved relationships between fossil turtle clades.

INTRODUCTION

The vertebrate head is the primary body part for sensory perception and interaction with the environment. Over the course of the last two centuries, two systems, the cranial nerve 
innervation and the cranial arterial circulation, have been studied extensively across extant and extinct tetrapods (e.g., Bojanus, 1819-21; Haughton, 1929; Shishkin, 1968; Lawson, 1970;

Starck, 1979; Evans, 1987; O’Keefe, 2001; Müller, Sterli \& Anquetin, 2011; Pardo \& Anderson, 2016). The cranial innervation system provides sensory cues to the brain while the carotid circulation system provides blood to sensory and other organs. Therefore, differences in cranial blood supply and innervation can give clues about the sensory capacities of animals (e.g., mechanoreception; Dehnhardt \& Mauck, 2008; Muchlinski, 2008; George \& Holliday, 2013), their physiology (e.g., thermoregulation; Porter \& Witmer, 2015; Yu, Ashwell \& Shulruf, 2019), the presence and type of cranial soft tissues in fossils (e.g., Benoit et al., 2018), as well as the evolution of these features along stem-lineages (e.g., Benoit, Manger \& Rubidge, 2016; Benoit et al., 2018; Joyce et al., 2018; Evers, Barrett \& Benson, 2019). Despite showing specific adaptations that often relate to ecological specializations, both the nervous and carotid systems are relatively conservative between major amniote clades in terms of their morphological evolution (Kardong, 1998; Müller, Sterli \& Anquetin, 2011) and can therefore be used to examine variational patterns along deep diverging lineages, possibly providing phylogenetic character support for specific divergences. Importantly, arteries and nerves are commonly housed in bony canals for at least parts of their courses, and can thus be identified and analyzed in specimens that lack soft tissues, such as dry museum specimens, or fossils. Therefore, phylogenetic approaches often focused on indirect evidence for the arterial circulation and cranial innervation, namely the respective bony canals, which can readily be compared across large specimen samples, including fossils. The difficulty here lies with the correct identification (and thus, homology) of said canals. Within turtles, variation pertaining to the carotid arterial system has been used as a source of phylogenetic characters since the seminal study of Gaffney 
58 (1975). On a gross scale, differences have been found between early shelled stem-turtles such as

59 Proganochelys quenstedtii from the Late Triassic (Gaffney, 1990) and crown-group turtles (e.g.,

60 Sterli \& de la Fuente, 2010; Rabi et al., 2013). Within crown Testudines, the two major surviving

61 clades, Pleurodira and Cryptodira, have been found to have different carotid circulation patterns

62 (Albrecht, 1967, 1976; Gaffney, 1975, 1979). Additionally, systematic differences between

63 cryptodires and pleurodires have been observed for the facial nerve system, which, for turtles,

64 appears to have experienced greater evolutionary change over time than other cranial nerves

65 (Gaffney, 1975, 1979). Parts of the facial nerve share the same course as parts of the carotid

66 arterial system (Albrecht, 1967, 1976; Gaffney, 1979; Miyashita, 2012). The shared course of

67 these systems potentially offers an explanation for the relatively greater variability of the facial

68 nerve system. In the past, most descriptive turtle studies have focused on either carotid

69 circulation (e.g., Sterli \& de la Fuente, 2010) or cranial innervation (e.g., Evers, Barrett \&

70 Benson, 2019) of single species, but seldom on both (Miyashita, 2012). Additionally, the

71 evolutionary understanding of the circulation system has been hampered by misidentifications

72 resulting from interpreting the carotid arterial system without explicit reference to the facial

73 nerve system, especially in fossils. This is exemplified by Eubaena cephalica, in which a canal

74 for the palatine artery had been persistently identified, but for whom CT scans have recently

75 shown that this canal was actually absent and that the only canal present in that area is the canal

76 for the vidian nerve (Rollot, Lyson \& Joyce, 2018).

\section{An overview of cranial carotid arterial circulation}

The main branching patterns of the carotid arterial circulation are conserved across amniotes (see

Figure 1 for a basic scheme of the cranial circulation and facial innervation of turtles). The 
81 internal carotid artery arises from the common carotid artery and splits into a stapedial branch

82 and a cerebral branch (e.g., Sedlmayr, 2002 for archosaurs including birds; Porter \& Witmer,

832015 for squamates; Porter, Sedlmayr \& Witmer, 2016 for crocodilians; and Müller, Sterli \&

84 Anquetin, 2011 for an amniote overview including stem-group taxa). In turtles, the cerebral

85 branch retains the name 'internal carotid artery' (e.g., McDowell, 1961; Gaffney, 1979; Sterli et

86 al., 2010; Rabi et al., 2013). The cerebral/internal carotid artery variously splits into anteriorly

87 directed arterial branches ('palatine artery' in turtles: e.g., Gaffney, 1979) (e.g., Sedlmayr, 2002;

88 Porter \& Witmer, 2015), but otherwise enters and traverses the basicranium through the

89 basisphenoid to exit into the pituitary fossa/sella turcica to supply blood to the brain (Sedlmayr,

90 2002; Müller, Sterli \& Anquetin, 2011; Porter \& Witmer, 2015; Porter, Sedlmayr \& Witmer,

91 2016). Additional anteriorly directed arteries, such as the infraorbital artery, may also branch off

92 the cerebral artery within the braincase (e.g., Porter \& Witmer, 2015).

93 Despite this conserved pattern of splitting into subordinate arteries, homology has not been

94 perfectly translated into nomenclature, which makes its understanding difficult. For instance, in

95 squamates and turtles, the artery that supplies the dorsal head region splits extracranially into two

96 major branches, one of which is the stapedial artery. The other branch enters the basicranium and

97 splits again into two subordinate arteries, one that supplies blood to the brain, and one that

98 typically supplies the facial region. In the turtle literature, the term 'internal carotid artery' is

99 used to refer to the arterial section that lies between the branching point of the stapedial artery,

100 and the intracranial split (e.g., McDowell, 1961; Gaffney, 1979; Sterli et al., 2010; Rabi et al.,

101 2013). Thus, in turtles, the 'internal carotid artery' branches into (i) the 'cerebral artery' that

102 traverses the basisphenoid and enters the pituitary fossa to supply the brain with blood, and (ii)

103 the 'palatine artery' that traverses the basicranium anteriorly toward the facial region. In 
104 squamates, however, the term 'internal carotid artery' is used for the carotid section prior to the 105 divergence of the stapedial artery (Oelrich, 1956; Porter \& Witmer, 2015). The second branch

106

107

108

109

110

111

112

113

114

115

116

117

118

119

120

121

122

123

124

125

126

that forms at this divergence is called the 'cerebral artery'. The 'cerebral artery' of squamates (= 'internal carotid artery' of turtles) then branches into the 'sphenopalatine artery' (= 'palatine artery' of turtles), and keeps its name for the remainder of its course into the brain (= 'cerebral artery' of turtles).

In this study, we use the classic turtle nomenclature with regard to the splitting pattern of carotids, which gained influence primarily by the works of Albrecht $(1967,1976)$ and Gaffney $(1972,1979)$. Under this nomenclature, the internal carotid artery and stapedial artery diverge from one another in an extracranial position, with the stapedial artery entering the cavum acustico-jugulare. The mandibular artery is frequently developed as a branch of the stapedial artery, although many exceptions exist across turtles. For instance, the mandibular artery derives from the internal carotid artery in trionychids (Albrecht, 1967). The internal carotid artery extends medially toward the basicranium and commonly, but not ubiquitously, splits into the cerebral and palatine arteries. The cerebral artery is always present and always traverses the basisphenoid and enters the sella turcica/pituitary fossa.

\section{A history of carotid research in turtles}

Early work on the carotid arterial system in turtles was mainly limited to the documentation of carotid artery courses in single species (e.g., Bojanus, 1819-21; Nick, 1912; Shindo, 1914), or a selection of related turtles (McDowell, 1961). Later, studies by Albrecht $(1967,1976)$ were the first synthetic approaches to summarize variation of features related to carotid circulation across clades. Albrecht's findings were further publicized by the landmark studies of Gaffney, who 
127 introduced this character complex to turtle phylogenetics (e.g., Gaffney, 1975; Gaffney \&

128 Meylan, 1988), and who advocated for the use of osteological correlates of the arteries, namely

129 the canals and foramina, to identify carotid features in fossil turtles, particularly by providing

130 terminology (Gaffney, 1972) and comparisons across a large sample of turtles (Gaffney, 1979).

131 The importance of the cranial circulatory system in systematics has been highlighted now for

132 more than half a century and many studies have added and/or revised characters related to

133 carotids (e.g., Gaffney, 1975; Meylan \& Gaffney, 1989; Jamniczky, 2008; Sterli \& de la Fuente,

134 2010; Sterli et al., 2010; Rabi et al., 2013; Zhou \& Rabi, 2015; Joyce et al., 2018; Evers \&

135 Benson, 2019; Hermanson et al., 2020), although methods of character implementation have

136 significantly varied between studies (e.g., Joyce, 2007; Anquetin, 2012; Evers \& Benson, 2019).

137 Several 'patterns' of circulation have been proposed, and other evolutionary scenarios have been

138 put forward. For instance, Meylan \& Gaffney (1989) postulated the cerebral and palatine arteries

139 of turtles primitively had a similar size and were slightly smaller than the stapedial artery.

140 Gaffney (1975) also proposed that the three major groups of turtles recognized at the time (i.e.

141 pleurodires, cryptodires, and paracryptodires) each display a characteristic pattern for the

142 circulation system, with the discriminating feature being the position of the foramen posterius

143 canalis carotici interni, i.e., the foramen through which the internal carotid artery enters the skull.

144 However, most of these inferences were based on only a small sample of dissected taxa obtained

145 during earlier studies (e.g., Albrecht, 1967, 1976), and patterns have been extrapolated from the

146 few taxa studied to entire turtle groups (Gaffney, 1979; Jamniczky \& Russell, 2007), without

147 further verification.

148 Paleontologists have been reporting patterns of cranial arterial circulation and innervation for

149 extinct species. These studies recognized that the observed features cannot easily be fit into the 
150 framework established on the few extant turtles studied in the past. For example, although all

151 crown-group turtles have their carotid arterial system fully embedded within the basicranium,

152 fully or partially ventrally-open carotid systems appear in stem-group turtles, but also on the

153 stem-lineages of various crown-group clades (e.g., Meylan \& Gaffney, 1989; Sterli et al., 2010;

154 Rabi et al., 2013). Rabi et al. (2013) proposed an updated, comprehensive nomenclatural system

155 for the diversity of foramina and canals associated with the carotid system, but this nomenclature

156 was not universally applied in recent turtle literature, despite its advantages. However, although

157 this contribution figuratively cut the Gordian knot of confusing terminology by suggesting an

158 internally consistent nomenclature, we here suggest two minor modifications to the names of the

159 canals that contain the cerebral and palatine arteries (see Material and Methods below). The

160 advent of CT-based studies has further led to a re-investigation of variation on a broad

161 taxonomic and sampling scale of extant and extinct turtles (e.g., Paulina-Carabajal et al., 2017;

162 Evers \& Benson, 2019; Hermanson et al., 2020), challenging some of the more classic character 163 concepts regarding the carotid system.

165 Facial nerve

166 Much less recent research has been done on the facial nerve (CN VII), despite the availability of 167 a broad historic literature describing nerve patterns in extant turtles (e.g., Bojanus, 1819-21;

168 Vogt, 1840; Kesteven, 1910; Gaupp, 1888; Hoffmann, 1890; Ruge, 1897; Siebenrock, 1897;

169 Bender, 1906; Noack, 1906; Ogushi, 1911, 1913a, 1913b; Hanson, 1919; Shiino, 1913; Nick, 170 1912; Shindo, 1914; Fuchs, 1931; van der Merwe, 1940; Soliman, 1964). The facial nerve

171 branches off the brain of turtles and exits the braincase via the fossa acustico-facialis on the

172 medial surface of the prootic (Fig. 1). From this fossa, it extends laterally through a canal. 
173 Further laterally, the facial nerve forms the geniculate ganglion, from which two major nerves

174 emerge, the anteriorly directed vidian/palatine nerve, and the posteriorly directed hyomandibular

175 nerve (Gaffney, 1979). Based on the comprehensive works of Siebenrock (1897), Shiino (1913),

176 Soliman (1964), and Gaffney $(1975,1979)$ suggested that the position of the geniculate ganglion

177 of the facial nerve differs systematically between cryptodires and pleurodires, with cryptodires

178 having the geniculate ganglion positioned in the canal for the lateral head vein, i.e. the canalis

179 cavernosus, and pleurodires having the geniculate ganglion contact the internal carotid artery

180 canal (=canalis caroticus internus). Gaffney's (1979) interpretation was based on relatively few

181 specimens, but has largely remained unchallenged and been used in many phylogenetic studies

182 of turtles. Although deviations to Gaffney's (1979) clear-cut patterns have been known for a long

183 time (e.g., Gaffney, 1983), a more recent, systematic survey of CT scans reveals many more,

184 including a potentially novel position for the geniculate ganglion (Evers and Benson, 2019). As

185 historically terms were used interchangeably in the past, Rollot, Lyson \& Joyce (2018) provided

186 a consistent nomenclature for the canals left by the facial nerve system. Here, we investigate the

187 facial nerve pattern on a broad taxonomic scale, and investigate how it is related to the carotid

188 arterial pattern, with which it partially shares canals (Miyashita, 2012).

189

190 Aims and objectives

191 For this contribution, we aim to document the carotid circulation pattern, as well as the course of

192 the facial nerve, for all major clades of extant turtles, with the explicit goal of wishing to clarify

193 if these structures can be traced correctly based on osteological material alone, in particular

194 fossils. Our work is primarily based on high-resolution micro-computed tomography (CT) scans

195 of 65 extant turtle species. The identity of canals was interpreted with reference to documented 
196 soft tissues (Albrecht 1967, 1976) whenever this information was available. We focus on the

197 position of foramina, the bones that form the canals and foramina, and the connections between

198 these structures (i.e., canals). In addition to updating the standardized terminology for these

199 features, we address the variation of these systems within and between clades and summarize

200 patterns common to specific clades. We provide evidence for the repeated loss of some branches

201 of the carotid arterial system and re-interpret canals that have been said to house the palatine

202 artery in these turtles as actually housing the vidian nerve, a branch of the facial nerve. Our work

203 provides an important step towards a comprehensive understanding of cranial arterial circulation

204 and facial nerve patterns, which can aid in character construction for phylogenetic analyses.

205 Furthermore, with the inclusion of fossil data, our work will provide the basis for a

206 comprehensive evolutionary understanding of cranial arterial circulation and innervation in

207 turtles.

208

209

MATERIAL AND METHODS

210

\section{CT-scanning and segmentation}

211 For this study, we broadly sampled across the turtle tree to compile a set of 69 CT scans of the

212 skulls of 65 extant turtle species (see Table 1). The relationships between the major clades of

213 turtles are shown in Figure 2. The vast majority of scans were previously published and are

214 available at public repositories (Evers and Benson, 2018; Lautenschlager, Ferreira \& Werneburg,

215 2018; Evers, 2019). The models generated are available on Morphobank

216 (http://morphobank.org/permalink/?P3732). The (para)basisphenoid, the left pterygoid, the

217 carotid system, the canalis cavernosus, and the facial nerve system of 14 skulls representing the

218 primary clades ("families") of extant turtles were segmented manually using the brush and lasso 
219 and interpolation tools of Amira 6.4.0 (https://www.fei.com/software) and final models were

220 generated by exporting surfaces of the structures of interest.

221

222

Assessment of size relationships between 'medial' and stapedial blood flow

223

Albrecht (1976) hypothesized that reductions in the size of the stapedial artery of turtles are

224 counterbalanced by increases in size of the palatine or orbital arteries ('medial' blood flow) to ensure the arterial blood supply for the facial region of the head. In order to quantitatively and statistically assess this hypothesis, we measured the cross-sectional areas of the stapedial and carotid canals in Dragonfly 4.0 (https://www.theobjects.com/dragonfly/index.html) (see Table

2). For the internal carotid artery canal, the cross sectional area was measured close to the foramen posterius canalis carotici interni. For the stapedial, palatine, and cerebral artery canals, the cross sectional area was measured close to their respective exit foramina. In all cases, measurements were taken orthogonal to the orientation of the canal. These measurements provide approximate estimates of blood flow, as arterial canal diameter is proportional to arterial size in turtles (Albrecht, 1976; Jamnicky and Russell, 2004). To test if the reduction of the stapedial artery has an effect on the 'medial' blood flow, we performed phylogenetic generalized least squares regression (pGLS; Grafen, 1989; Rohlf, 2001) of the $\log _{10}$-transformed internal carotid arterial cross-sectional area on the respective measurement for the stapedial artery. pGLS is a modification of ordinary least squares regression (OLS), which takes into account the expected co-variance structure of residuals that is the result of phylogenetic non-independence of the input data (e.g., Felsenstein, 1985; Grafen, 1989; Symonds \& Blomberg, 2014). The expected variances and co-variances of traits among species follow a Brownian motion model of trait evolution in pGLS and related methods like phylogenetic independent contrasts (e.g., 
242 Felsenstein, 1985; Pagel, 1997, 1999), although different models of trait evolution can be used in

243 pGLS. The strength of the phylogenetic correlation was estimated under maximum likelihood

244 during the model fitting using variable Pagel's lambda (Revell, 2010; Motani \& Schmitz, 2011).

245 Pagel's lambda is a parameter that describes the model of evolution, with values of 1 indicating

246 that trait evolution occurred under Brownian motion, whereas a value of 0 means there is no

247 phylogenetic signal in the residuals of the regression. pGLS was performed in R with the $g l s()$

248 function of the package ape (Paradis \& Schliep, 2018), and the corPagel option for setting the

249 correlation structure of the model. Because of phylogenetic correlation in the error structure of a

250 pGLS, the coefficient of correlation $\mathrm{R}^{2}$ cannot be easily defined for phylogenetic regressions

251 (Ives, 2018). Here, we use a generalized form of $\mathrm{R}^{2}$ described by Nagelkerke (1991), which is

252 derived by comparison of the log-likelihood of the model with that of a null model $\left(\log _{10}(\mathrm{CCI}) \sim\right.$

253 1). Because results from our initial pGLS analysis suggest that chelonioids, trionychians, and

254 kinosternoids show differences in regression slope to other turtles (see results), we performed

255 generalized least-squares phylogenetic analysis of covariance (pANCOVA) using the gls.ancova

256 function of the package evomap (Smaers \& Rohlf, 2016; Smaers \& Mongle, 2018) to test if our

257 data can be better explained by a model with different intercepts or slopes or both for the visually

258 separated groups. We defined a group with consistently positive residuals (i.e. chelonioids,

259 kinosternoids, trionychians) and another with the remaining taxa for which models could be

260 compared. As the phylogenetic input for the pGLS analyses, we used the dated phylogeny of

261 Pereira et al. (2017), which we pruned to match our taxon sample. Two species in our sample,

262 Kinixys erosa and Pseudemys floridana, were not included in the study of Pereira et al. (2017).

263 We re-assigned the tip-labels of two closely related species, Kinixys belliana and Pseudemys

264 texana, in the tree of Pereira et al. (2017) to those of our sampled species. This procedure 
265

assumes that Kinixys erosa (or Pseudemys floridana) is closer related to Kinixys belliana (or Pseudemys texana) than to any other turtle in the tree, which is justified by the circumstance that the monophyly of neither the species of Kinixys nor Pseudemys are disputed, and by the fact that we sampled only one species of each genus in our study. For the regression analysis, we chose the internal carotid artery as representing 'medial' blood flow, as opposed to the combined values of the cerebral and palatine arteries. This allowed us to include taxa for which the palatine artery has no osteological correlate but is present (i.e. chelonioids; see Results). Additionally, this procedure allowed the inclusion of podocnemidids. Although podocnemidids provide no reliable estimation for the cross-sectional area of the internal carotid artery, as the artery extends through the enlarged cavum pterygoidei (see Results), these turtles could be included by taking the crosssectional areas of the cerebral artery instead. This can be justified, as the palatine artery is absent in podocnemidids (see Results), and thus the cerebral artery canal represents the internal carotid artery in terms of cross-sectional area (=blood flow). Although interesting for questions we would have liked to address, we had to exclude Dermatemys mawii and Kinosternon scorpioides from the analysis $(n=2)$ because of their completely reduced stapedial arteries, as the logarithm of zero is undefined. Additionally, we excluded Kinosternon baurii after an initial run of analyses including it. $K$. baurii has a strongly reduced stapedial artery size in absolute numbers (two orders of magnitude smaller than in other kinosternids), and has a proportionally extremely large internal carotid artery (same order of magnitude as in other kinosternids), leading to an extreme positive residual when included into a regression. It can, therefore, be statistically defined as an outlier with large leverage on the regression line. Although the data for K. baurii strongly supports the notion that internal carotid artery size is strongly increased when the 
287 stapedial artery size is strongly reduced (see Discussion), we conservatively excluded the taxon.

288 Our sample in total was thus $n=63$.

289

290

\section{Nomenclature and Homology}

291

As our primary sources of information are skulls, we were not able to observe the cranial

292

293

294

295

296

297

298

299

300

301

302

303

304

305

306

307

308

309

circulation and innervation systems directly, but rather could only assess their former presence

by explicit reference to the canals they left in bone in combination with published dissections, in particular Albrecht $(1967,1976)$. The vast majority of our assessments are, nevertheless, uncontroversial. We therefore shorten our descriptions by not justifying explicitly why we believe a particular canal to be filled by a particular soft structure. Instead, we only provide separate justifications in the text for the rare cases where our conclusions differ from previously published ones.

The dissections of Albrecht $(1967,1976)$ documented strong variation in the organization of the cranial blood irrigation of turtles, which are interchangeably supplied by branches of the internal carotid artery and the stapedial artery. For instance, the sensory organs and tissues of the snout region (i.e., "the palate") are mostly supplied by the stapedial branch in chelydrids, testudinoids, and pleurodires, by the palatine branch in kinosternoids, by a mixture of the stapedial and palatine branches in chelonioids, but by the orbital branches of the cerebral branch in trionychids. Conversely, the mandible is supplied by the stapedial branch in chelydrids, pleurodires, and testudinoids, but by the palatine branch in kinosternoids, chelonioids, and trionychids. So, while the terms "cerebral" and "stapedial" are somewhat misleading, as they disguise the fact that tissues other than the brain and stapes are often supplied by them, the term "palatine" is positively misleading, as the relevant artery, if present, does not supply the palate at 
310 all in many turtles. The situation is especially problematic in trionychids, where the "palatine

311 artery" only supplies the mandible, while the palate is entirely supplied by a large subordinate

312 branch of the "cerebral artery."

313 The nomenclatural system of Rabi et al. (2013) created nomenclatural clarity by providing

314 separate names for all possible canals and openings formed by the internal carotid system, but

315 accidentally introduced a new source of confusion by selecting names for the canals that negate

316 the homology of the vessels that they contain. As the system is still relatively new, we here take

317 the opportunity to provide minor adjustments, by replacing the terms canalis caroticus cerebralis

318 with canalis caroticus basisphenoidalis and canalis caroticus palatinum with canalis caroticus

319 lateralis. We select these terms as they have historic precedence. The term canalis caroticus

320 basisphenoidalis connects to the term "foramen caroticum basisphenoidale," which was

321 introduced by Gaffney (1983) for the posterior opening of the carotid canal that punctures the

322 basisphenoid (e.g., the internal carotid canal of Gaffney, 1983, but the cerebral canal of Rabi et

323 al., 2013). Although this term was sometimes applied to the fenestra caroticus of Rabi et al.

324 (2013) (e.g., Meylan \& Gaffney, 1989; Brinkman \& Peng, 1996; Sukhanov, 2000), it was mostly

325 used for the aforementioned posterior opening in subsequent descriptions of basal turtles (e.g.,

326 Gaffney, 1990; Gaffney \& Meylan, 1992; Gaffney and Ye, 1992; Brinkman \& Peng, 1993;

327 Gaffney, 1996; Sukhanov, 2000). The term canalis caroticus lateralis, on the other hand, had

328 been used consistently over the course of the entire 20th century (Gaffney, 1972, 1979), but had

329 been replaced purposefully by Rabi et al. (2013), as the name of the canal did not make reference

330 to the arteries it contained. However, as highlighted above, the replacement term "palatine canal"

331 is just as confusing, as the vessels held in this canal do not supply the palate in many turtles. In

332 addition to reconnecting to the historic literature, these newly established names have the 
333 advantage of being purely positional and not implying what vessels they contain. This

334 nomenclature therefore only highlights the homology that exists to the basic branching pattern

335 seen in the carotid system of turtles. As the artery held in the canalis caroticus basisphenoidalis

336 universally supplies the brain, we here retain the term "cerebral artery," even though other

337 structures may be supplied as well by subordinate branches that diverge off the cerebral artery

338 within the sella turcica. For the artery held in the canalis caroticus lateralis, we will try to be

339 specific, where possible, by reference to Albrecht $(1967,1976)$. However, in anticipation of the

340 needs of paleontologists, who cannot know what tissues are supplied by the arteries passing

341 through the lateral canal, we suggest the informal term "lateral branch of the internal carotid

342 canal."

343 In addition to the modifications provided for the nomenclature of the cranial arterial

344 circulation system, we also add the terms 'proximal' and 'distal' to some aspects of the

345 innervation system. Although the usage of 'proximal' and 'distal' are unusual in a cranial

346 context, we find these terms useful to refer to distinct sections of nerves within the cranium. The

347 'proximal' part of a nerve is close to the brain or its ganglion, whereas 'distal' portions are close

348 to the innervated tissue. Although the sensory nerves discussed herein pass information from the

349 sensory organs or innervated tissues to the brain, we follow Gaffney $(1972,1979)$ by describing

350 sensory nerve canals as moving away from the brain, to remain consistent with the literature.

351 Our modified list for the definitions of all canals we discuss and criteria on how to recognize

352 them is as follows:

353 Cavum cranii - We here restrict the meaning of the term cavum cranii from referring to the

354 entire space from the fossa nasalis to the foramen magnum (Gaffney, 1972) to the endocranial

355 space limited anterodorsally by the sulcus olfactorius and posteriorly by the foramen magnum. 
Canalis caroticus internus - The bony canal that contains the main branch of the internal

357 carotid artery from the foramen posterius canalis carotici interni to the fenestra caroticus (only developed in some fossil turtles) or its split into the canalis caroticus lateralis and canalis caroticus basisphenoidalis (Rabi et al., 2013). Whenever the split of the internal carotid artery cannot be identified, typically because of the absence of a formed canalis caroticus lateralis, the canalis caroticus internus is defined as the portion of the canal that does not yet traverse the basisphenoid, as opposed to the canalis caroticus basisphenoidalis, which is defined as the portion of the canal that is located inside the basisphenoid. Additional structures might be contained in the canalis caroticus internus, in particular the vidian nerve, but this is not reflected in the name. The canalis caroticus internus typically traverses the skull at the junction of the basisphenoid with the pterygoid and/or prootic in the posterior prolongation of the canalis caroticus basisphenoidalis.

Foramen posterius canalis carotici interni - The posterior foramen to the canalis caroticus internus, which serves as the entry of the internal carotid artery into the skull (Rabi et al., 2013).

Canalis caroticus basisphenoidalis - The canal that contains the cerebral artery from the

371 fenestra caroticus (only developed in some fossil turtles) or from the split of the canalis caroticus

372 internus into its primary branches to the foramen anterius canalis carotici basisphenoidalis

373 (canalis caroticus cerebralis of Rabi et al., 2013). Whenever a canalis caroticus lateralis cannot

374 be identified, the canalis caroticus basisphenoidalis is defined as the portion of the carotid canal

375 that traverses the basisphenoid, as opposed to the more posteriorly located canalis caroticus

376 internus, which is located outside of the basisphenoid. No other structures are known to traverse

377 this canal. The canalis caroticus basisphenoidalis typically penetrates the basisphenoid at mid- 
378 length and universally exits the basisphenoid within the sella turcica. The canalis caroticus

379 basisphenoidalis is the canal with the greatest diameter to pierce the basisphenoid.

Foramen anterius canalis carotici basisphenoidalis - The anterior opening of the canalis

381

382

caroticus basisphenoidalis (foramen anterius canalis carotici cerebralis of Rabi et al., 2013), universally located within the sella turcica of the basisphenoid.

Canalis caroticus lateralis - The bony canal that contains the lateral branch of the internal carotid canal, typically the palatine artery, from the fenestra caroticus (only developed in some fossil turtles) or from the the split of the canalis caroticus internus into its two primary branches to the foramen anterius canalis carotici lateralis (canalis caroticus palatinum of Rabi et al., 2013). The vidian nerve may traverse part of this canal. The canalis caroticus lateralis typically punctures the skull at the junction of the basisphenoid with the pterygoid and exits within the sulcus cavernosus and near the anterior end of the latter.

\section{Foramen anterius canalis carotici lateralis - The anterior exit of the canalis caroticus} lateralis, which typically contains the palatine artery, located near the anterior end of the sulcus cavernosus (foramen anterius canalis carotici palatinum of Rabi et al., 2013).

Canalis carotico-pharyngealis - A single or series of ventrally-directed bony canals in the pterygoid that contain the arteria carotico-pharyngealis. The arteria carotico-pharyngealis splits from the palatine artery, enters the canalis carotico-pharyngealis to extend ventrally through the pterygoid, and exits the skull through the foramen carotico-pharyngeale (Albrecht, 1967). We only infer the presence of a canalis carotico-pharyngealis whenever a canal connects the lateral canal with the ventral surface of the pterygoid.

Foramen carotico-pharyngeale - The ventral exit of the canalis carotico-pharyngealis located on the ventral surface of the pterygoid. 
Canalis cavernosus - The bony canal that contains the lateral head vein and extends between

402

403

404

405

406

407

408

the foramen cavernosum anteriorly and the cavum acustico-jugulare posteriorly (Gaffney, 1972).

The canalis cavernosus is usually formed by the quadrate, prootic, and pterygoid. The distal portion of the hyomandibular nerve may traverse this canal as well. Additionally, the mandibular artery may traverse this canal in some turtle clades. The canalis cavernosus is generally a straight canal with a large diameter that obliquely traverses the skull lateral to the braincase.

Canalis nervus facialis - The bony canal that contains the facial nerve medial to its split at the geniculate ganglion into the vidian and hyomandibular branches (Rollot, Lyson \& Joyce, 2018). The canal is recognized as the structure laterally penetrating the prootic from the fossa acustico-facialis. It may end within the canalis cavernosus, the canalis caroticus internus, or at its split into the vidian and hyomandibular canals.

\section{Canalis nervus hyomandibularis proximalis - The bony canal that is laterally continuous} with the canalis nervus facialis, and joins the canalis cavernosus. This canal contains the proximal part of the hyomandibular branch of the facial nerve, but is only present in turtles in which the geniculate ganglion is not positioned within the canalis cavernosus.

Canalis nervus hyomandibularis distalis - The bony canal that originates within the wall of the canalis cavernosus posterior to the connection of the canalis nervus facialis or canalis nervus hyomandibularis proximalis. The canal extends posteriorly, paralleling the canalis cavernosus, and joins the cavum acustico-jugulare. It contains the distal portion of the hyomandibular branch of the facial nerve, and is only present in some turtles.

Canalis pro ramo nervi vidiani - The bony canal that contains the posterior (=proximal) portion of the vidian nerve to the exclusion of the other primary nerves and blood vessels defined herein (Rollot, Lyson \& Joyce, 2018). In living turtles, the canal, when present, connects the 
424 geniculate ganglion to the canalis caroticus internus and traverses the prootic and pterygoid 425 along its way.

426 Canalis nervus vidianus - The bony canal that contains the anterior (=distal) portion of the

427 vidian nerve to the exclusion of the other primary nerves and blood vessels defined herein

428 (Rollot, Lyson \& Joyce, 2018). The canal typically originates from the canalis caroticus lateralis 429 and anteroposteriorly traverses the pterygoid or palatine to open towards the foramen palatinum 430 posterius. Numerous accessory canals may branch off this canal that innervate the dorsal or 431 ventral side of the pterygoid or palatine.

432 Foramen anterius canalis nervi vidiani - The anterior exit of the canalis nervus vidianus 433 (Rollot, Lyson \& Joyce, 2018). The foramen is typically located posterior to the foramen 434 palatinum posterius, but can be split into multiple foramina arranged on the dorsal or ventral 435 surfaces of the pterygoid or palatine.

436 Foramen arteriomandibulare - A separate, anterior exit for the mandibular artery from the 437 canalis cavernosus into the temporal fossa. It is located just posterior to the foramen nervi 438 trigemini.

RESULTS

\section{Chelidae (Fig. 3)}

442 Canalis caroticus internus - In all chelids studied, the internal carotid artery enters the skull ventrally. The foramen posterius canalis carotici interni (Figs. 3A-3B) is formed by the prootic

444 in Phrynops hilarii and Hydromedusa tectifera, by the basisphenoid and the prootic in Chelus 445 fimbriatus, by the prootic and quadrate in Chelodina oblonga and Phrynops geoffroanus, and by 446 the basisphenoid, prootic and quadrate in Emydura subglobosa. Historically, only the proootic 
447 and basisphenoid were recognized as contributing to the foramen in chelids (Siebenrock, 1897;

448 Albrecht, 1976). However, recent phylogenetic studies have documented further variation, such

449 as the contribution of the quadrate (Evers \& Benson, 2019). Albrecht (1976) similarly described

450 the foramen posterius canalis carotici interni as only being formed by the prootic and

451 basisphenoid in the chelids he studied. The apparent variation in bone contributions to this

452 foramen was recently coded by Evers and Benson (2019) as separate characters. The canalis

453 caroticus internus is directed anteromedially and formed by the prootic and basisphenoid in

454 Chelus fimbriatus (Figs. 3A-3B), P. hilarii, and H. tectifera, by the quadrate, prootic, and

455 basisphenoid in Chelodina oblonga and P. geoffroanus, and by the quadrate, prootic,

456 basisphenoid and pterygoid in E. subglobosa. Again, this contrasts with Albrecht (1976), who

457 noted that the canalis caroticus internus is only formed by the prootic and basisphenoid. We are

458 able to observe a canal extending anteriorly along the pterygoid-basisphenoid suture in

459 Chelodina oblonga and $H$. tectifera, which fits with the expected position of a canalis caroticus

460 lateralis. A canal in a more lateral position is found in Chelus fimbriatus (Figs. 3A-3B), E.

461 subglobosa, P. geoffroanus, and P. hilarii. Albrecht (1976) stated that the canalis caroticus

462 lateralis is present in all the chelids he observed, except for Chelus fimbriatus, and Hermanson et

463 al. (2020) also identified a canalis caroticus lateralis in chelids. A close reading of Albrecht

464 (1976) reveals that he did not observe the palatine or any other artery in any chelid in his sample

465 and that the vidian nerve is the sole structure to occupy the canalis caroticus lateralis instead.

466 These observations are indirectly confirmed by our sample, as the canal in question does not split

467 from the canalis caroticus internus in Chelodina oblonga (right side) and H. tectifera, but rather

468 directly from the inferred position of the geniculate ganglion itself, following on from the

469 position of the latter and extending anteriorly mostly along the pterygoid-basisphenoid suture 
470 (see Fig. 3A for illustration as Chelus fimbriatus exhibits a similar configuration). The

471 dissections of Albrecht (1976), clearly showing the presence of the vidian nerve and absence of

472 the palatine artery, and our observations for Chelodina oblonga and H. tectifera, support the fact

473 that the canal of interest very likely corresponds to the canalis nervus vidianus and should not be

474 termed canalis caroticus lateralis. The case of chelids is a good example to highlight how

475 identification of canals can be tricky and misleading when osteological correlates are the only

476 source of information available. As Albrecht (1976) provided dissection-based descriptions, we

477 were able to decide on the identity of the canals with confidence, without which we would have

478 identified the canal extending along the pterygoid-basisphenoid suture as the canalis caroticus

479 lateralis, as the latter is expected to be located at this position in most turtles. The case

480 highlighted herein shows how much carefulness is required when working on the cranial

481 circulation, especially in fossils, for which soft tissues are only rarely preserved (see Discussion).

482 The canalis carotico-pharyngealis is absent. The canalis caroticus basisphenoidalis, herein

483 defined by its entry into the basisphenoid due to the absence of the canalis caroticus lateralis, and

484 the foramen anterius canalis carotici basisphenoidalis are formed by the basisphenoid (Figs. 3A-

485 3C). The foramina anterius canalis carotici basisphenoidalis are widely-spaced (Figs. 3A-3B).

486 The canalis stapedio-temporalis is the largest canal in all observed chelids, ranging from ten

487 times bigger than any carotid canal in P. hilarii, to two times the size of them in E. subglobosa.

488 The canalis caroticus internus is the smallest canal in Chelodina oblonga, E. subglobosa, and $P$.

489 hilarii, whereas the canalis caroticus basisphenoidalis is the smallest in Chelus fimbriatus, $H$.

490 tectifera, and P. geoffroanus (Table 2). 
Mandibular artery - There is no trace in bone of the mandibular artery in our sample. This

492 is consistent with Albrecht's (1976) conclusion that the (internal) mandibular artery of chelids branches from the stapedial artery after its exit from the foramen stapedio-temporale.

Canalis cavernosus - The canalis cavernosus (Figs. 3A-3D) extends from the foramen cavernosum to the level of the foramen stapedio-temporale in Chelus fimbriatus, $P$. hilarii and $P$. geoffroanus, but posterior to the level of the foramen stapedio-temporale in Chelodina oblonga, E. subglobosa, and H. tectifera. The canalis cavernosus is formed by the quadrate, prootic, and basisphenoid in Chelodina oblonga, by the prootic, pterygoid, basisphenoid, and parietal in Chelus fimbriatus, by the quadrate and prootic in E. subglobosa, and by the quadrate, prootic, and pterygoid in $P$. hilarii, $P$. geoffroanus, and $H$. tectifera. The foramen cavernosum itself is formed by the prootic and pterygoid. In Chelus fimbriatus, the lateral head vein is fully enclosed by bone a second time anterior to the foramen nervi trigemini, a feature not observed in other chelids (Figs. 3A-3D). In all observed chelids but E. subglobosa, the canalis cavernosus connects to a large ventrolaterally directed canal that opens on the ventral surface of the pterygoid, and which we call the 'chelid canal' herein (Figs. 3A-3B). Siebenrock (1897) observed this canal in all chelids and interpreted it as containing a branch of the carotid artery, but dissections made by Albrecht (1976) on Chelodina sp. and Mesoclemmys nasuta showed that the canal only contains connective tissue.

Facial nerve canal system - The facial nerve extends laterally from the cavum cranii to the canalis caroticus internus through the canalis nervus facialis, which is formed by the prootic

511 (Figs. 3A, 3D). The geniculate ganglion is inferred to be in contact with the canalis caroticus

512 internus in all observed chelids (Figs. 3A, 3D). The hyomandibular branch extends

513 posterolaterally through the prootic in its own canal, the canalis nervus hyomandibularis 
514 proximalis, for most of its length (Figs. 3A-3D). In Chelodina oblonga and E. subglobosa, the

515 quadrate contributes to the canalis nervus hyomandibularis proximalis posteriorly. In all

516 observed taxa but E. subglobosa, the canalis nervus hyomandibularis proximalis connects to the

517 canalis cavernosus posteriorly, close to the position of the columella auris. In E. subglobosa,

518 however, the canalis nervus hyomandibularis proximalis does not connect to the canalis

519 cavernosus and directly joins the cavum acustico-jugulare. As already discussed above, Albrecht

520 (1976) suggested that the palatine artery is absent and that the vidian nerve extends through the

521 canalis caroticus lateralis, but we here conclude based on the same observations that the lateral

522 carotid canal is absent and that the vidian nerve traverses the canalis nervus vidianus. In Chelus

523 fimbriatus, the vidian nerve emerges from the geniculate ganglion and does not enter the canalis

524 caroticus internus, but rather directly enters the canalis nervus vidianus which crosses the prootic

525 and pterygoid to join the sulcus cavernosus at the level of the foramen nervi trigemini (Figs. 3A,

526 3D). The path is generally similar in other chelids, but in $P$. hilarii, $P$. geoffroanus, and $H$.

527 tectifera, the vidian nerve partially enters the canalis caroticus internus, whereas in Chelodina

528 oblonga and E. subglobosa the vidian nerve fully enters the canalis caroticus internus before

529 splitting from it again further anteriorly. The vidian nerve passes for this part of the vidian canal

530 through the prootic, pterygoid, and basisphenoid in Chelodina oblonga and H. tectifera, through

531 the prootic and pterygoid in Chelus fimbriatus, E. subglobosa, $P$. hilarii, and $P$. geoffroanus. The

532 basisphenoid contribution to this canal had previously been noted for Chelus fimbriatus and

533 Chelodina longicollis by Siebenrock (1897). In E. subglobosa, P. hilarii, P. geoffroanus, and $H$.

534 tectifera, no trace of the vidian nerve is visible inside the bone anterior to the foramen nervi

535 trigemini, so the vidian nerve probably lies inside the sulcus cavernosus. In Chelus fimbriatus,

536 the vidian nerve re-enters the skull anteriorly through the lateral wall of the sulcus cavernosus 
537 and extends anteriorly along the pterygoid-parietal suture for a long distance and at its anterior

538 end, splits into two short canals that leave the skull posterolaterally to the descending process of

539 the parietal (Figs. 3A-3B, 3D). The foramen anterius canalis nervi vidiani is formed by the

540 pterygoid and parietal. A similar pattern is visible in Chelodina oblonga, except that the portion

541 of the vidian nerve that is enclosed by bone is much shorter.

542

543 Podocnemididae (Fig. 4)

544 Canalis caroticus internus - The main branch of the internal carotid artery is never tightly

545 enclosed by bone in the observed podocnemidids, so a true foramen posterius canalis carotici

546 interni is absent, at least if a tight enclosure is used as a definitional criterion (Fig. 4A). Instead,

547 podocnemidids possess an enlarged cavity, the podocnemidoid fossa (Lapparent de Broin \&

548 Werner, 1998) or cavum pterygoidei (Gaffney, Tong \& Meylan, 2006), through which the carotid

549 artery extends and that is additionally filled by muscle tissue (Albrecht, 1976). As the internal

550 carotid artery is not enclosed by bone, it is difficult to make clear statements regarding the

551 circulation pattern of the carotid artery in our specimens. We can only identify with confidence a

552 short canalis caroticus basisphenoidalis that is formed by the basisphenoid (Fig. 4A). These

553 observations broadly agree with those of Siebenrock (1897), though with different nomenclature,

554 as he interprets the entry of the cavum pterygoidei as the foramen posterius canalis carotici interni.

555 Albrecht (1976) described a small artery splitting from the internal carotid artery in Podocnemis

556 sextuberculata that he hypothesized to be a possible, vestigial palatine artery, but he was not able

557 to identify similar structures in the other podocnemidids he dissected. This small artery splits into

558 two branches, one supplying the muscle inside the cavum pterygoidei, one joining the canalis

559 cavernosus to supply tissue within the sulcus cavernosus. Albrecht (1976) also noted the presence 
560 of the foramen anterius canalis carotici lateralis in all of the podocnemidids he observed. We 561 identify a large, irregularly shaped fenestra between the cavum pterygoidei and the sulcus 562 cavernosus in both specimens of Podocnemis unifilis that may correspond to the foramen anterius 563 canalis carotici lateralis of Albrecht (1976). Given that Albrecht (1976) only observed a blood 564 vessel that passes this fenestra in one out of four podocnemidids in his sample and that this vessel 565 does not supply tissues related to the palate, we conclude that the palatine artery is not present in 566 extant podocnemidids. The canalis carotico-pharyngealis are absent. The foramina anterius canalis 567 carotici basisphenoidalis are widely-spaced (Figs. 4A-4B). The canalis stapedio-temporalis is the 568 largest canal, being five times larger than the canalis caroticus basisphenoidalis in one of our 569 specimens of Podocnemis unifilis (NHMUK 60.4.16.9), but only twice as large in the other 570 (FMNH 45657) (Table 2).

571 Mandibular artery - We are not able to find any traces of a mandibular artery in our sample 572 of podocnemidids. This is consistent with Albrecht's (1976) observation that the external and 573 internal mandibular arteries of podocnemidids branch off the stapedial artery prior to and after its 574 passage through the canalis stapedio-temporalis, respectively.

575 Canalis cavernosus - The canalis cavernosus (Figs. 4A-4D) extends posteriorly from the 576 foramen cavernosum to the level of the foramen stapedio-temporale in all podocnemidids and is 577 formed by the quadrate, prootic, and pterygoid. The foramen cavernosum is formed by the prootic 578 and pterygoid.

579 Facial nerve canal system - The facial nerve canal is short and extends mediolaterally through 580 the prootic (Figs. 4A, 4D). At mid-distance between the canalis cavernosus and the fossa acustico581 facialis, i.e. within the prootic, the facial nerve canal bifurcates into two branches, indicating the 582 position of the geniculate ganglion and the split of the facial nerve into the hyomandibular and 
583 vidian branches (Figs. 4A, 4D). In our first specimen of Podocnemis unifilis (NHMUK 60.4.16.9),

584 the hyomandibular branch extends posterolaterally through the prootic through a canalis nervus

585 hyomandibularis proximalis. This canal coalesces with the canalis cavernosus (Figs. 4A, 4D). For

586 most of its distal course along the canalis cavernosus, the hyomandibular nerve extends along a

587 sulcus in the wall of the canalis cavernosus. However, the sulcus becomes a proper canalis nervus

588 hyomandibularis distalis in the posterior-most section of the canalis cavernosus, just before

589 entering the cavum acustico-jugulare (Fig. 4B). In our second specimen of Podocnemis unifilis

590 (FMNH 45657), a short but distinct canalis nervus hyomandibularis distalis joining the cavum

591 acustico-jugulare is present. In our first specimen of Podocnemis unifilis (NHMUK 60.4.16.9), the

592 vidian nerve extends ventrally through the canalis pro ramo nervi vidiani, which is formed by the

593 prootic, to enter the cavum pterygoidei (Figs. 4A, 4C-4D). The vidian nerve is then inferred to

594 first extend through the cavum pterygoidei to then pierce the full anteroposterior length of the

595 pterygoid, where it splits into several branches (Fig. 4D). These branches extend through the

596 pterygoid, palatine, and parietal, and exit the skull through various foramina formed by these bones

597 (Figs. 4A-4D). The path is inferred to be similar for the other specimen of Podocnemis unifilis

598 (FMNH 45657), but the vidian nerve first crosses the above-mentioned fenestra to enter the sulcus

599 cavernosus before entering the pterygoid and only splits into two branches within that bone. The

600 lateral one extends through the pterygoid and its anterior foramen is formed by the pterygoid and

601 the palatine. The medial one extends through the pterygoid, palatine, and parietal, and its anterior

602 foramen is formed by the palatine and parietal. Siebenrock (1897) noted for Erymnochelys

603 madagascariensis that the vidian nerve exits the prootic posteromedially to cross the cavum

604 pterygoidei (his canalis caroticus internus), and then enters the pterygoid, traverses it in its own

605 canal, and exits the pterygoid on its dorsal surface, posterior to the orbit and medial to foramen 
606 palatinum posterius. Albrecht (1976) noticed that the vidian nerve mainly extends through the 607 pterygoid as well.

608

609 Pelomedusidae (Fig. 5)

610 Canalis caroticus internus - In Pelomedusa subrufa and Pelusios subniger, the carotid artery 611 enters the skull from ventral and the foramen posterius canalis carotici interni (Fig. 5A-5C) is 612 formed by the basisphenoid and the prootic. The internal carotid artery is directed anteromedially 613 and extends through the prootic and the basisphenoid. The canalis caroticus internus intersects 614 with the facial nerve system at the inferred position of the geniculate ganglion dorsally in the 615 prootic close to the foramen posterius canalis carotici interni (Figs. 5A, 5D). This intersection with 616 the facial nerve system is superficial so that the vidian nerve never fully enters the canalis caroticus 617 internus (Figs. 5A, 5D). The intersection occurs in the prootic in Pelusios subniger and along the 618 prootic-basisphenoid suture in Pelomedusa subrufa. This slightly differs from Albrecht's (1976) 619 observations, who noted that this intersection occurred only in the prootic in both species. In 620 Pelomedusa subrufa, the intersection is exposed ventrally. Albrecht (1976) also noted the absence 621 of the palatine artery and foramen anterius canalis carotici lateralis in dissected specimens of 622 Pelomedusa subrufa and Pelusios subniger. As we do not observe any canal in the expected 623 position of the canalis caroticus lateralis, we here confirm observations of Albrecht (1976) and 624 conclude that the canalis caroticus lateralis is absent in pelomedusids due to the absence of the 625 palatine artery. For the same reason, the canalis carotico-pharyngealis is absent as well. The canalis 626 caroticus basisphenoidalis, herein defined as beginning with the entry of the carotid system into 627 the basisphenoid, and foramen anterius canalis carotici basisphenoidalis are formed by the 628 basisphenoid (Fig. 5A). The foramina anterius canalis carotici basisphenoidalis are widely-spaced 
629 (Fig. 5A). The canalis stapedio-temporalis is the largest canal, being four times larger than the 630 canalis caroticus internus and canalis caroticus basisphenoidalis in Pelomedusa subrufa, and about 631 three times larger than them in Pelusios subniger (Table 2).

632 Mandibular artery - Albrecht (1976) documented that the mandibular artery of pelomedusids 633 branches from the stapedial artery after its passage through the stapedio-temporal canal. Evers \& 634 Benson (2019), on the other hand, reported the presence of a foramen arteriomandibulare for 635 Pelomedusa subrufa, which suggests passage of the mandibular artery through the sulcus 636 cavernosus. After restudying the same CT scans as used by Evers \& Benson (2019), however, we 637 support the initial conclusion of Albrecht (1976), as the reported foramen arteriomandibulare 638 appears to be an ontogenetic artifact (see Discussion for more details).

639 Canalis cavernosus - The canalis cavernosus (Figs. 5A-5D) extends posteriorly from the 640 foramen cavernosum to the level of the foramen stapedio-temporale in Pelomedusa subrufa, but 641 posterior to the foramen stapedio-temporale in Pelusios subniger. In Pelusios subniger, the 642 canalis cavernosus extends posterolaterally through the prootic, whereas in Pelomedusa subrufa, 643 the quadrate contributes to the lateral border of the canalis cavernosus for much of its path. The 644 foramen cavernosum is formed by the prootic in both species.

645 Facial nerve canal system - The facial nerve extends laterally from the braincase toward the 646 canalis caroticus internus through the prootic (Figs. 5A, 5D). The geniculate ganglion is inferred 647 to contact the canalis caroticus internus in both species and the canalis pro ramo nervi vidiani is 648 consequently absent (Figs. 5A, 5D). In Pelusios subniger, a long canalis nervus hyomandibularis 649 proximalis is present and directed posterolaterally through the prootic (Figs. 5A-5D). The 650 canalis nervus hyomandibularis proximalis joins the ventral part of the canalis cavernosus at the 651 level of the bifurcation between the canalis cavernosus and canalis stapedio-temporalis. In 
652 Pelomedusa subrufa, the hyomandibular branch almost directly enters the canalis cavernosus and 653 is therefore mainly visible as a sulcus alongside the wall of the latter. At the level of the contact

654 between the geniculate ganglion and the internal carotid artery, the vidian nerve leaves the 655 canalis caroticus internus. In Pelusios subniger, the split of the facial nerve occurs near the 656 dorsal part of the canalis caroticus internus and the vidian nerve directly enters a separate canalis 657 nervus vidianus (Figs. 5A, 5D). This canal first shortly extends through the prootic and along the 658 suture formed between the prootic, pterygoid, and basisphenoid. The vidian nerve then continues 659 anteriorly through the pterygoid and the foramen anterius canalis nervi vidiani is formed by the 660 pterygoid (Figs. 5A-5D). In Pelomedusa subrufa, the split of the facial nerve occurs on the 661 dorsolateral part of the canalis caroticus internus and for a short distance, the vidian nerve 662 extends through the canalis caroticus internus. The vidian nerve then branches off the canalis 663 caroticus internus and extends out of the skull, ventral to the prootic and basisphenoid, before re664 entering the skull through the pterygoid via a canalis nervus vidianus. The vidian nerve then 665 extends anteriorly through the pterygoid, and the foramen anterius canalis nervi vidiani is formed 666 by the pterygoid.

667

\section{Carettochelyidae (Fig. 6)}

669 Canalis caroticus internus - The internal carotid artery enters the skull close to the posterior end of the basisphenoid and the foramen posterius canalis carotici interni (Figs. 6A-6B) is formed

671 by the pterygoid only. The canalis caroticus internus extends anteromedially mostly through the 672 pterygoid, and enters the basisphenoid, at which point it transforms into the canalis caroticus 673 basisphenoidalis, considering that the lateral carotid canal is absent (Figs. 6A-6B). Albrecht 674 (1976) reported that the foramen anterius canalis carotici lateralis is either absent or small, but did 
675 not draw any firm conclusions about a potentially lacking palatine artery as he only had access to

676 skeletal material. CT scans of a stained specimen available to us however show that the only canal

677 splitting from the canalis caroticus internus contains the vidian nerve, which allows us to conclude

678 that the palatine artery and canalis caroticus lateralis are absent. The canalis pro ramo nervi vidiani

679 joins the canalis caroticus internus halfway along its path (Figs. 6B, 6D). Somewhat anterior to

680 this connection, just before the origin of the canalis caroticus basisphenoidalis, and along the

681 pterygoid-basisphenoid suture, the vidian nerve leaves the canalis caroticus internus laterally to

682 form the vidian canal (Figs. 6A-6B, 6D). The canalis carotico-pharyngealis is absent. The canalis

683 caroticus basisphenoidalis is formed by the basisphenoid. The foramina anterius canalis carotici

684 basisphenoidalis are widely separated from each other and formed by the basisphenoid (Fig. 6A).

685 The canalis stapedio-temporalis, canalis caroticus internus, and canalis caroticus basisphenoidalis

686 are relatively large and roughly equal-sized (Table 2).

687 Mandibular artery - Joyce, Volpato \& Rollot (2018) previously noted the absence of the 688 canalis caroticus lateralis and speculated by reference to trionychids, where the mandibular artery 689 passes through the lateral carotid canal, that the mandible of Carettochelys insculpta may be 690 supplied by the cerebral artery instead. CT scans of the aforementioned stained specimen, 691 however, clarify that the mandible of Carettochelys insculpta is supplied by the mandibular artery, 692 which branches off the stapedial artery and extends through the canalis cavernosus and the 693 posteriorly elongated trigeminal foramen (Fig. 6D). The only recently reported foramen 694 arteriomandibulare apparent in carettochelyids (Evers and Benson, 2019) serves as an excellent 695 osteological correlate.

696 Canalis cavernosus - The canalis cavernosus (Figs. 6A-6D) is very short and extends 697 posteriorly from the foramen cavernosum to the level of the foramen stapedio-temporale. For most 
698 of its path, the canalis cavernosus is bordered by the quadrate laterally and ventrolaterally, and by

699 the prootic dorsally, medially and ventromedially. Slightly posterior to the foramen cavernosum,

700 the pterygoid minorly contributes to the ventral limit of the canalis cavernosus. The foramen

701 cavernosum is formed by the quadrate, prootic, and pterygoid.

702 Facial nerve canal system - The facial nerve course of Carettochelys insculpta was already

703 described in detail by Joyce, Volpato \& Rollot (2018) based on the same specimen and CT scans.

704 The facial nerve extends laterally from the cavum cranii through the prootic. At two-thirds of the

705 distance between the fossa acustico-facialis and the canalis cavernosus, the canalis nervus facialis

706 splits into canals for the vidian and hyomandibular branches (Figs. 6A, 6D). The aforementioned

707 stained specimen confirms a geniculate ganglion position within the prootic, although the position

708 is close to the wall of the canalis cavernosus and not as deeply within the prootic as in many

709 pleurodires. The hyomandibular nerve extends laterally along its own proximal canal through the

710 prootic for a short distance and joins the canalis cavernosus, from which it further extends

711 posteriorly within a sulcus (Figs. 6A, 6D). The canalis pro ramo nervi vidiani is small, extends

712 through the prootic and pterygoid, and connects to the canalis caroticus internus halfway along its

713 length (Figs. 6B, 6D). The vidian nerve leaves the canalis caroticus internus laterally at the

714 basisphenoid-pterygoid suture (Figs. 6A-6B, 6D). Its proximal part is located in the pterygoid, the

715 most distal portion fully in the palatine. The canalis nervus vidianus ends in a series of various

716 small canals that open on the dorsal and ventral surfaces of the palatine (Figs. 6A-6B, 6D).

718 Trionychidae (Fig. 7)

719 Canalis caroticus internus - In all observed taxa, the internal carotid artery enters the skull

720 from ventral and the foramen posterius canalis carotici interni (Figs. 7A-7C) is formed by the 
721 pterygoid only. The canalis caroticus internus (Figs. 7A-7D) is formed by the pterygoid and

722 basisphenoid in all trionychid specimens with minor contributions of the prootic in Amyda

723 cartilaginea, Apalone mutica, Apalone spinifera, Chitra indica, Cyclanorbis senegalensis, and

724 Pelodiscus sinensis. Jamniczky \& Russell (2007) only identified the basisphenoid and pterygoid

725 as contributing to the canalis caroticus internus in Ap. mutica and Trionyx triunguis. The

726 differences in observation for Ap. mutica may be due to intraspecific variation or error in

727 observation. Many other such differences exist for other taxa and structures. As these

728 possibilities can only be tested in the context of much greater sampling, we here assume that all

729 such differences pertain to intraspecific variation, while favoring our observations in the tables.

730 In Am. cartilaginea, Chitra indica, P. sinensis, and Cycloderma frenatum, the internal carotid

731 artery is exposed dorsally, i.e. in the floor of the cavum acustico-jugulare, for a short distance

732 between the foramen posterius canalis carotici interni and the canalis pro ramo nervi vidiani. The

733 canalis pro ramo nervi vidiani enters the canalis caroticus internus midway and the vidian nerve

734 follows the path of the canalis caroticus lateralis (Fig. 7D). In Cycloderma frenatum, the vidian

735 nerve splits directly from the canalis caroticus internus instead of following the path of the

736 canalis caroticus lateralis. The split of the internal carotid artery into its two subbranches, in this

737 case the cerebral and mandibular arteries (Albrecht, 1967), occurs at the same level as the sella

738 turcica (Figs. 7A-7B). The canalis caroticus lateralis and canalis caroticus basisphenoidalis are

739 short. Indeed, the canalis caroticus lateralis is shortened to the length of a fenestra. The

740 mandibular artery joins the sulcus cavernosus through this fenestra, which is formed by the

741 pterygoid and basisphenoid in Am. cartilaginea, Ap. mutica, Ap. spinifera, Chitra indica, and $P$.

742 sinensis, but only by the pterygoid in Cyclanorbis senegalensis, Cycloderma frenatum and $L$.

743 punctata. The canalis carotico-pharyngealis is absent, as observed by Albrecht in Trionyx spp. 
744 (1967). In all examined trionychids, the cerebral branch exits through the sella turcica. The

745 foramina anterius canalis carotici basisphenoidalis are widely-spaced and formed by the

746 basisphenoid (Fig. 7B). The canalis caroticus basisphenoidalis is larger than the canalis stapedio-

747 temporalis and canalis caroticus lateralis in all observed trionychids. However, some differences

748 as to which canal is the smallest are apparent across taxa. The canalis caroticus lateralis is the

749 smallest in Am. cartilaginea, C. indica, and Cycloderma frenatum, whereas the canalis stapedio-

750 temporalis is the smallest in Ap. mutica, Cyclanorbis senegalensis, L. punctata, and P. sinensis.

751 The canalis stapedio-temporalis and canalis caroticus lateralis are equal-sized in Ap. spinifera

752 (Table 2). This corroborates Albrecht (1976), who noted that trionychids have a small stapedial

753 artery and a large carotid artery, but slightly differs from Jamniczky \& Russell (2007), who

754 identified a foramen anterius canalis carotici lateralis that is larger than the foramen anterius

755 canalis carotici basisphenoidalis in Ap. mutica and Trionyx triunguis. The structures we identify

756 in trionychids are identical to the ones identified by Albrecht $(1967,1976)$.

757 Canalis cavernosus - The canalis cavernosus (Figs. 7A-7D) extends from the foramen

758 cavernosum to the level of the foramen stapedio-temporale or slightly posterior to it. The canalis

759 cavernosus is formed by the pterygoid, prootic, and quadrate in all observed taxa except

760 Cycloderma frenatum, in which the canalis cavernosus is only bordered by the prootic and

761 pterygoid. The foramen cavernosum is formed by the prootic and pterygoid.

762 Facial nerve canal system - The facial nerve extends laterally from the fossa acustico-

763 facialis through the prootic (Figs. 7A, 7D). The geniculate ganglion is inferred to be located in

764 the canalis cavernosus in Am. cartilaginea, Ap. mutica, Ap. spinifera (Figs. 7A, 7D), and $P$.

765 sinensis, but in between the canalis cavernosus and canalis caroticus internus in Chitra indica,

766 Cyclanorbis senegalensis, Cycloderma frenatum, and L. punctata. This observation contradicts 
767 Gaffney (1979), who stated that the geniculate ganglion is always in contact with the canalis

768 cavernosus in cryptodires. The facial nerve splits into two branches in all observed trionychids

769 but Cyclanorbis senegalensis, the ventral one being the vidian nerve, and the lateral one being

770 the hyomandibular branch (Fig. 7D). In Cyclanorbis senegalensis, a third branch is present that

771 extends anteriorly along the prootic-pterygoid suture to join the sulcus cavernosus. The distal

772 portion of the hyomandibular branch is fully confluent with the canalis cavernosus in $\mathrm{Am}$.

773 cartilaginea, is present as a sulcus connected to the canalis cavernosus in Ap. spinifera, Ap.

774 mutica, and $P$. sinensis, and extends posterolaterally through its own canalis nervus

775 hyomandibularis proximalis along the prootic-pterygoid suture in Chitra indica, Cyclanorbis

776

777

778

779

780

781

782

783

784

785

786

787

788

789

senegalensis, Cycloderma frenatum and L. punctata. In all observed trionychids, the vidian nerve is contained in the canalis pro ramo nervi vidiani and extends ventrally from the geniculate ganglion through the prootic and pterygoid to join the canalis caroticus internus midway (Fig.

7D). In all taxa but Cycloderma frenatum, the vidian branch likely initially follows the course of the canalis caroticus internus, but then passes through the fenestra-like canalis caroticus lateralis to enter the sulcus cavernosus. From there, the nerve pierces either the pterygoid or the palatine to form the canalis nervus vidianus (Figs. 7A, 7D). In Cycloderma frenatum, the vidian branch leaves the canalis caroticus internus laterally between the canalis pro ramo nervi vidiani and canalis caroticus lateralis and then joins the sulcus cavernosus. Bender (1906) described a similar pattern in Apalone ferox, noting that the vidian nerve enters a canal formed by the basisphenoid and pterygoid (our canalis caroticus internus) and extends anteriorly along the suture made by these two bones. The vidian nerve then bifurcates, extends anteriorly along the pterygoidpalatine suture, and connects with the maxillary branch of the trigeminal nerve $\left(\mathrm{V}_{2}\right)$ outside of the bone to form the sphenopalatine ganglion, at the level of the contact between the pterygoid 
790 and the maxilla. Our observations for trionychids in general correspond to those of Ogushi

791 (1911) for P. sinensis and Shiino (1913) for the vidian nerve in Am. cartilaginea. The canalis

792 nervus vidianus is formed by the palatine in Ap. spinifera, Chitra indica, P. sinensis, and

793 Cyclanorbis senegalensis, by the pterygoid and palatine in Ap. mutica, Am. cartilaginea and $L$.

794 punctata, and by the pterygoid, palatine and parietal in Cycloderma frenatum. In Ap. spinifera, 795 Am. cartilaginea, and P. sinensis, the canalis nervus vidianus splits into several smaller canals 796 that exit the skull on the dorsal and ventral surfaces of the palatine. Much of this variation was 797 previously noted by Meylan (1987) for a broader sample. The foramen anterius canalis nervi 798 vidiani is formed by the palatine in all observed trionychids.

799

800 Kinosternidae (Fig. 8)

801 Canalis caroticus internus - In all observed kinosternids, the internal carotid artery initially

802 enters the skull through the fenestra postotica and then passes through a groove within the cavum 803 acustico-jugulare that is roofed by the prootic, as previously noted by Evers and Benson (2019).

804 The actual foramen posterius canalis carotici interni (Figs. 8A-8C) is formed by the pterygoid 805 and prootic, and the canalis caroticus internus (Figs. 8A-8B) is formed by the pterygoid, prootic, 806 and basisphenoid. This slightly differs from the observations of Siebenrock (1897), who did not 807 mention the involvement of the basisphenoid in the formation of the canalis caroticus internus. 808 Jamniczky \& Russell (2007) only mentioned the pterygoid as forming the foramen posterius 809 canalis carotici interni, and the basisphenoid and pterygoid as forming the canalis caroticus 810 internus in Kinosternon baurii and Staurotypus salvinii. The canalis pro ramo nervi vidiani

811 connects to the canalis caroticus internus just before its split into its palatine and cerebral

812 branches (Albrecht, 1976; Figs. 8B, 8D). The canalis caroticus lateralis and canalis caroticus 
813 basisphenoidalis are short. The canalis caroticus lateralis joins the sulcus cavernosus. The canalis

814 caroticus lateralis and foramen anterius canalis carotici lateralis are formed by the prootic,

815 pterygoid, and basisphenoid in Sternotherus minor, Kinosternon subrubrum, and Staurotypus

816 salvinii, by the pterygoid and basisphenoid in $K$. baurii, and only by the pterygoid in

817 Kinosternon scorpioides. Two canalis carotico-pharyngealis are present in K. scorpioides and

818 Staurotypus salvinii. The cerebral branch exits at the sella turcica. The canalis caroticus

819 basisphenoidalis and foramen anterius canalis carotici basisphenoidalis are formed by the

820 basisphenoid. The foramina anterius canalis carotici basisphenoidalis are widely separated (Fig.

821 8A). The canalis caroticus lateralis has a greater cross-section than the canalis caroticus

822 basisphenoidalis and canalis stapedio-temporalis in all observed kinosternids, and the canalis

823 stapedio-temporalis, which is absent in $K$. scorpioides, is the smallest canal in K. baurii, $K$.

824 subrubrum, Staurotypus salvinii, and Sternotherus minor (Table 2). With the exception of $K$.

825 baurii, for which Jamniczky and Russell (2007) were not able to identify the canalis stapedio-

826 temporalis and foramen stapedio-temporalis, the relative canal sizes we observe in our specimens

827 confirm the observations of McDowell (1961), Albrecht (1967, 1976), and Jamniczky \& Russell

828 (2007).

829 Mandibular artery - The mandibular artery (Fig. 8D) branches from the palatine artery

830 within the sulcus cavernosus and exits through the trigeminal foramen (Albrecht, 1967). It is

831 therefore not surprising that we cannot find any osteological correlates for this artery beyond the

832 enormous size of the anterior foramen of the lateral carotid canal.

833 Canalis cavernosus - The canalis cavernosus (Figs. 8A-8D) extends posteriorly from the

834 foramen cavernosum and joins the cavum acustico-jugulare slightly posterior to the level of the 
835 foramen stapedio-temporale. It is formed by the quadrate, prootic, and pterygoid. The foramen

836 cavernosum is formed by the prootic and pterygoid.

837 Facial nerve canal system - The facial nerve extends ventrolaterally from the cavum cranii to

838 the canalis cavernosus through the prootic (Figs. 8A, 8D). The geniculate ganglion is inferred to

839 be located in the canalis cavernosus and splits into two branches in all observed kinosternids (Figs.

$8408 \mathrm{~A}, 8 \mathrm{D})$. The hyomandibular nerve extends posterolaterally within the canalis cavernosus, visible

841 as a sulcus in K. subrubrum, K. baurii, K. scorpioides, and Sternotherus minor (Figs. 8A, 8D), but

842 undistinguishable from the canalis cavernosus in Staurotypus salvinii. In all observed kinosternids,

843 the vidian nerve is contained in a short canalis pro ramo nervi vidiani that extends ventrally through

844 the prootic and pterygoid to join the canalis caroticus internus (Figs, 8B, 8D). In Sternotherus

845 minor, $K$. subrubrum, $K$. baurii, and $K$. scorpioides, the vidian nerve extends within the canalis

846 caroticus internus and then anteriorly within the canalis caroticus lateralis, from which it exits into

847 the sulcus cavernosus. Slightly more anteriorly in the sulcus cavernosus, approximately at the level

848 of the dorsum sellae of the basisphenoid, the vidian nerve enters its own canal (Figs. 8A-8D). This

849 canal, the canalis nervus vidianus, is formed by the pterygoid and palatine, with some contributions

850 of the parietal in K. baurii, and of the epipterygoid in K. scorpioides. Within the palatine, the vidian

851 nerve splits into several branches. One of these branches extends to the foramen palatinum

852 posterius, while the others either become untraceable in the porosity of the bone, or connect to the

853 dorsal or ventral surfaces of the palatine (likely forming, respectively, the foramina arteriae

854 anteriovidianae and foramina arteriaevidianae of Albrecht, 1967). In Staurotypus salvinii, a canal

855 leaves the canalis caroticus internus ventrolaterally and merges with a canal coming from ventral.

856 This complex then becomes confluent with another canal coming from the canalis caroticus

857 internus, and the resulting canal divides more anteriorly into two branches. The largest one extends 
858 anterodorsally and joins the sulcus cavernosus anterior to the foramen anterius canalis carotici

859 lateralis. The smallest one extends anteriorly through the pterygoid and palatine, likely 860 corresponding to the canal for the vidian nerve. The vidian nerve exits the skull on the

861 ventromedial side of the palatine, posterior to the anterior end of the pterygoid. More anteriorly,

862 the vidian nerve likely re-enters the skull, merges with unidentified canals, and exits near the

863 foramen palatinum posterius inside the palatine. The pattern we observe for the vidian nerve differs

864 in two respects from the descriptions of McDowell (1961) and Albrecht (1967). Firstly, McDowell

865 (1961) only mentioned the pterygoid as the bone forming the vidian canal, whereas we observed

866 a higher complexity of the innervation pattern, with the palatine, parietal, and epipterygoid

867 contributing to the formation of the canalis nervus vidianus. Secondly, Albrecht (1967) noted that

868 some branches of the vidian nerve leave the canalis pro ramo nervi vidiani to extend anteriorly

869 through the pterygoid, and anteriorly connect with other branches of the vidian nerve that exit the

870 canalis caroticus lateralis. Although we cannot exclude that Albrecht (1967) indeed observed that

871 in the specimens he dissected, we have not been able to observe this pattern in our specimens and

872 the canalis nervus vidianus is either exclusively connected to the sulcus cavernosus (Sternotherus

873 minor, K. subrubrum, K. baurii, and K. scorpioides), or the canalis caroticus internus (Staurotypus

874 salvinii).

875

876 Dermatemydidae (Fig. 9)

877 Canalis caroticus internus - In Dermatemys mawii, the internal carotid artery enters the skull 878 through the fenestra postotica. The posterior course of the internal carotid artery can be inferred

879 from a dorsally open trough in the pterygoid. The internal carotid artery only becomes fully 880 enclosed by bone within the cavum acustico-jugulare, where the prootic covers the pterygoid to 
881 form the actual foramen posterius canalis carotici interni (Fig. 9A). The internal carotid artery is

882 bordered by the prootic, pterygoid, and basisphenoid. The canalis pro ramo nervi vidiani enters

883 the internal carotid canal about mid-length. Slightly posterior to the split into the canalis caroticus

884 lateralis and canalis caroticus basisphenoidalis, a small canal of unclear function or homology

885 connects the canalis caroticus internus to the canalis cavernosus on the right side of the available

886 specimen. The split of the canalis caroticus internus into the canalis caroticus basisphenoidalis and

887 canalis caroticus lateralis occurs along the pterygoid-basisphenoid suture (Figs. 9A-9B). The

888 canalis caroticus lateralis is short and formed by the prootic, pterygoid, and basisphenoid. The

889 herein contained palatine artery (Albrecht, 1976) joins the sulcus cavernosus by the way of the

890 foramen anterius canalis carotici lateralis, which is formed by the pterygoid and basisphenoid

891 (Figs. 9A-9B). A canalis carotico-pharyngealis is absent. The canalis caroticus basisphenoidalis

892 extends anteromedially through the basisphenoid and joins the braincase via the widely-spaced

893 foramina anterius canalis carotici basisphenoidalis, which are formed by the basisphenoid (Fig.

894 9A). The canalis caroticus lateralis is about four times bigger in cross section than the canalis

895 caroticus basisphenoidalis (Table 2), and the canalis stapedio-temporalis and foramen stapedio-

896 temporale are absent, possibly explaining the particularly large diameter of the canalis caroticus

897 lateralis.

898 Mandibular artery - We are unaware of any literature that explicitly discusses the placement

899 of the mandibular artery in Dermatemys mawii. Its branching from the stapedial artery posterior to

900 the stapedial canal and exit through the trigeminal foramen is suggested by a lateral sulcus within

901 the canalis cavernosus. This sulcus is otherwise only apparent in turtles for which the mandibular

902 artery extends through the canalis cavernosus. However, the large diameter of the lateral carotid

903 canal, on the other side, suggests that the mandible is perhaps partially or fully supplied by the 
904 palatine artery as well. The taxa that phylogenetically bracket Dermatemys mawii do not provide 905 any further evidence, as the mandible is supplied by the stapedial artery in chelydrids, but by the 906 palatine artery in kinosternids. As we believe the division of the canalis cavernosus and the relative 907 size of the lateral carotid canal to be a useful osteological correlates, we reconstruct Dermatemys 908 mawii as having a split mandibular artery (Fig. 9D), as seen in Cheloniidae. This issue will need 909 to be resolved in the future, however, by reference to wet specimens.

910 Canalis cavernosus - The canalis cavernosus (Figs. 9A-9D) extends posteriorly from the

911 foramen cavernosum and joins the cavum acustico-jugulare slightly anterior to the level of the 912 columella auris. The canalis cavernosus is formed by the quadrate, prootic, and pterygoid. The 913 foramen cavernosum is formed by the prootic and pterygoid.

914 Facial nerve canal system - The facial nerve canal extends ventrolaterally from the cavum 915 cranii to the canalis cavernosus through the prootic (Figs. 9A, 9D). As there are no osteological 916 indications for the split of the facial nerve to occur prior to the facial nerve canal joining the canalis 917 cavernosus, the position of the geniculate ganglion is inferred to be within the latter (Figs. 9A, 918 9D). And as no osteological correlate for the hyomandibular branch of the facial nerve could be 919 identified within the canalis cavernosus, its course is inferred to be posteriorly directed within that 920 structure (Fig. 9D). A small canalis pro ramo nervi vidiani is visible on the right side only in the 921 available specimen that extends along the prootic-pterygoid suture and joins the canalis caroticus 922 internus mid-length. The vidian nerve is then inferred to follow the path of the canalis caroticus 923 internus and the canalis caroticus lateralis into the sulcus cavernosus (Fig. 9D). Anterior to the 924 rostrum basisphenoidale and posteroventromedially to the processus inferior parietalis, the vidian 925 nerve leaves the sulcus cavernosus ventrolaterally to enter the canalis nervus vidianus, extends 926 anteriorly through the palatine, and ends at the foramen palatinum posterius (Figs. 9A-9D). 
928 Chelydridae (Fig. 10)

929 Canalis caroticus internus - The internal carotid artery enters the skull slightly anterior to

930 the level of the columella auris through the fenestra postotica. The artery forms a dorsally

931 exposed sulcus within the cavum acustico-jugulare in Macrochelys temminckii, but not in

932 Chelydra serpentina. The foramen posterius canalis carotici interni (Fig. 10A) within the cavum

933 acustico-jugulare is formed by the prootic and pterygoid in C. serpentina, but only by the

934 pterygoid in M. temminckii, as noted by Siebenrock (1897). The canalis caroticus internus (Fig.

935 10B) is formed by the prootic, pterygoid, and basisphenoid. This slightly differs from Siebenrock

936 (1897) who only described the prootic and pterygoid as the bones forming this canal in $C$.

937 serpentina, and only the pterygoid in M. temminckii, and from Jamniczky \& Russell (2007), who

938 noted that the canalis caroticus internus traverses the pterygoid and basisphenoid in $C$.

939 serpentina. The canalis pro ramo nervi vidiani connects the canalis cavernosus to the canalis

940 caroticus internus about mid-length of the latter. In both observed chelydrids, the split of the

941 canalis caroticus internus into the canalis caroticus lateralis and canalis caroticus

942 basisphenoidalis occurs at the pterygoid-basisphenoid suture (Figs. 10A-10B). The canalis

943 caroticus lateralis is much longer than the canalis caroticus basisphenoidalis. The palatine artery

944 (Albrecht, 1976) extends anteriorly through the canalis caroticus lateralis, first through the

945 pterygoid and then along the pterygoid-basisphenoid suture in M. temminckii, but only along the

946 pterygoid-basisphenoid suture in C. serpentina (Figs. 10A-10D). The palatine artery then exits

947 its canal through the foramen anterius canalis carotici lateralis, formed by the pterygoid and

948 basisphenoid (Figs. 10A-10B). This contrasts with Albrecht (1976), who noted that the foramen

949 anterius canalis carotici lateralis is only formed by the pterygoid in chelydrids. A canalis 
950 carotico-pharyngealis is present in both taxa. The canalis caroticus basisphenoidalis extends

951 anteromedially through the basisphenoid and opens within the sella turcica. The foramina

952 anterius canalis carotici basisphenoidalis are widely-spaced in C. serpentina (Fig. 10A) but close

953 together in M. temminckii. The canalis stapedio-temporalis is the largest canal and the canalis

954 caroticus lateralis the smallest in terms of diameters in both specimens. The canalis stapedio-

955 temporalis is about five times larger than the canalis caroticus basisphenoidalis in C. serpentina,

956 and ten times larger in M. temminckii, and more than ten times larger than the canalis caroticus

957 lateralis in both specimens (Table 2). These canal proportions corroborate the results of

958 McDowell (1961), Albrecht (1976), and Jamniczky \& Russell (2007).

959 Mandibular artery - A dorsal groove within the large canalis cavernosus provides an

960 osteological correlate for the presence of two parallel lying vessels, which we interpret as the

961 ventromedially located lateral head vein and the dorsolaterally located mandibular artery, which

962 exits the canalis cavernosus through the trigeminal foramen (Albrecht, 1976; Figs. 10A-10B, 963 10D).

964 Canalis cavernosus - The canalis cavernosus (Figs. 10A-10D) extends from the foramen 965 cavernosum to the level of the foramen stapedio-temporale. It is short in C. serpentina, but long 966 in M. temminckii. The canalis cavernosus is formed by the quadrate, prootic, and pterygoid, and 967 houses the geniculate ganglion. The foramen cavernosum is formed by the prootic and pterygoid.

968 Facial nerve canal system - The facial nerve canal extends ventrolaterally from the fossa 969 acustico-facialis to the canalis cavernosus through the prootic (Figs. 10A, 10D). The geniculate 970 ganglion is inferred to be positioned within the canalis cavernosus and splits into two branches

971 (Figs. 10A, 10D). The hyomandibular branch has no osteological correlate in M. temminckii, but

972 its course remains visible in $C$. serpentina as a sulcus in the wall of the canalis cavernosus (Figs. 
$97310 \mathrm{~A}, 10 \mathrm{D})$. In $C$. serpentina, the canalis pro ramo nervi vidiani is short and located along the

974 prootic-pterygoid suture, whereas it is long in M. temminckii and formed by the pterygoid only.

975 The vidian nerve enters the internal carotid canal at mid-length in both taxa. In C. serpentina, the

976 vidian nerve leaves the canalis caroticus internus just posterior to the split of the canalis caroticus

977 internus into the canalis caroticus lateralis and canalis caroticus basisphenoidalis (Figs. 10A, 10D).

978 The canalis nervus vidianus is long but narrow in diameter, extends anteriorly through the 979 pterygoid, and connects to small exiting canals along its path (Figs. 10A-10D). The main vidian 980 canal opens onto the dorsal surface of the pterygoid, anterior to the foramen anterius canalis 981 carotici lateralis, and posteromedially to the anterior limit of the processus inferior parietalis (Figs.

982 10A-10D). Siebenrock (1897) described the vidian nerve of $C$. serpentina as extending through a 983 groove lateral to the internal carotid artery, being posterodorsally covered by the prootic, and 984 posteriorly starting from the foramen jugulare anterius, and that the vidian nerve extends through 985 the canalis caroticus lateralis and foramen anterius canalis carotici lateralis. Albrecht (1976) 986 similarly stated that the canal we identify as the vidian canal does not connect to the canalis 987 caroticus internus and that no nerve is contained in it. Our observations differ from these 988 descriptions as our specimen clearly exhibits a canalis nervus vidianus distinct from the canalis 989 caroticus lateralis and that is connected to the canalis caroticus internus, as previously suggested 990 by Gaffney (1972a) for this taxon, even though it is hard to distinguish and sometimes barely 991 visible in the CT scans. In M. temminckii, two small canals ventrally leave the canalis caroticus 992 internus near its posterior end and pass through the pterygoid to eventually merge through detours 993 with a canal that extends anteriorly through the pterygoid, lateral to the canalis caroticus internus, 994 to connect with a branch of the canalis carotico-pharyngeale, and to merge with the canalis 995 caroticus lateralis close to the foramen anterius canalis carotici lateralis. This system of canals may 
996 have held the vidian nerve. This conclusion differs from Albrecht (1976) who described the canalis

997 nervus vidianus in $M$. temminckii as connecting to the canalis caroticus internus and bifurcating

998 from it slightly posterior to the split of the canalis caroticus internus into the canalis caroticus

999 lateralis and canalis caroticus basisphenoidalis. His canalis nervus vidianus then extends anteriorly

1000 through the pterygoid and opens into the orbit at the anterior end of the crista pterygoidea.

1001

1002

\section{Cheloniidae (Fig. 11)}

1003

Canalis caroticus internus - In all observed cheloniids, the internal carotid artery enters the

1004

1005

1006

1007

1008

1009

1010

1011

1012

1013

1014

1015

1016

1017

1018

skull from posterior at the posteroventral part of the pterygoid. The foramen posterius canalis carotici interni (Figs. 11A-11B) is formed by the pterygoid. In Eretmochelys imbricata, Chelonia mydas, and Natator depressus, the canalis caroticus internus (Figs. 11A-11D) is formed by the pterygoid and basisphenoid, but only by the pterygoid in Lepidochelys olivacea and Caretta caretta. In E. imbricata and Chelonia mydas, a narrow and short canal leaves the canalis caroticus internus dorsally at about half of its length to join the cavum labyrinthicum. In E. imbricata, Chelonia mydas, and $N$. depressus, the canalis pro ramo nervi vidiani connects the canalis cavernosus to the canalis caroticus internus (Figs. 11A-11B, 11D), but in L. olivacea and Caretta caretta no canalis pro ramo nervi vidiani is visible. Unlike in all other turtles, the internal carotid artery exits the basicranium to enter the sulcus cavernosus prior to its split into subordinate arteries in all observed cheloniids (Figs. 11A, 11D). This had previously been inferred by Zangerl (1953) for Chelonia mydas and observed in dissected specimens of the same species by Albrecht (1976), and then observed for cheloniids more widely by Evers \& Benson (2019). The position of the canalis caroticus basisphenoidalis provides osteological evidence for this unusual arrangement. This canal usually diverges off the canalis caroticus internus within the basicranium and at the 
1019 basisphenoid-pterygoid suture. In cheloniids, however, the canalis caroticus basisphenoidalis 1020 diverges from the sulcus cavernosus into the basisphenoid (Figs. 11A, 11D). Therefore, a short 1021 part of the internal carotid artery must be inferred to extend dorsally uncovered within the sulcus 1022 cavernosus across all cheloniids. Consequentially, the palatine artery, the presence of which has 1023 been confirmed for at least Chelonia mydas by Albrecht (1976), is never encased in a canal in 1024 cheloniids (Fig. 11D), confirming observations by Evers \& Benson (2019), who reported this 1025 condition to be present in all extant cheloniids and Dermochelys coriacea, but not in all extinct cheloniids. A characteristic commonly used in turtle systematics (and first used explicitly by Shaffer et al., 1997) is the spacing of the foramina anterius canalis carotici basisphenoidalis, which in most turtles are widely spaced across the sella turcica, but come close together in cheloniids (Fig. 11A) and some extinct turtles, such as plesiochelyids (Gaffney, 1976). Recently, Evers \& Benson (2019) defined a third passage for the cerebral arteries, based on the observation that right and left arteries converge within the basisphenoid and exit through a single median foramen anterius canalis carotici basisphenoidalis in L. olivacea, Caretta caretta, and N. depressus, but not in other extant cheloniids. Here, we confirm these observations. As the palatine artery is never encased in a bony canal, the identification of the canals traversing the pterygoid as being the canalis carotico-pharyngealis is not fully certain. However, respectively one and two canals in $E$. imbricata and Caretta caretta connect the ventral surface of the pterygoid to the portion of the sulcus cavernosus in which the carotid artery lies, making them plausible candidates for being the canalis carotico-pharyngealis. The canalis stapedio-temporalis is about twice as large as the canalis caroticus internus and about four to five times larger than the canalis caroticus basisphenoidalis in all observed cheloniids (Table 2). Although we cannot infer the diameter of the palatine artery in 
1041 the absence of a formed canalis caroticus lateralis, observations made by Albrecht (1976) show

1042 that the palatine artery is larger than the cerebral artery.

1043 Mandibular artery - Albrecht (1976) reported that the mandible of chelonioids is supplied by

1044 two separate branches, a vestigial one originating from the stapedial artery and a larger one

1045 supplied by the palatine artery. We find osteological evidence of the latter in the form of a sulcus

1046 within the canalis cavernosus (Figs. 11A-11C), combined with a foramen arteriomandibulare that

1047 is present in Chelonia mydas, but not in other cheloniids. In contrast to Albrecht (1976), however,

1048 we note that the foramen arteriomandibulare does not form a short canal in C. mydas, but rather

1049 represents a small fenestra to the canalis cavernosus. In addition, the lateral sulcus within the

1050 canalis cavernosus in our specimen is suggestive of a large mandibular artery (Figs. 11A-11C).

1051 This is further supported by the dried remains of the mandibular artery in our specimen of

1052 Eretmochelys imbricata. Although further studies on wet specimens will need to further clarify

1053 this issue, we reconstruct chelonioids as having similarly sized anterior and posterior mandibular

1054 arteries (Fig. 11D).

1055 Canalis cavernosus - The canalis cavernosus (Figs. 11A-11D) extends from the foramen

1056 cavernosum to the level of the foramen stapedio-temporale. The canalis cavernosus is formed by

1057 the quadrate, prootic and pterygoid and the foramen cavernosum is formed by the prootic and 1058 pterygoid.

1059 Facial nerve canal system - The facial nerve canal extends ventrolaterally from the fossa 1060 acustico-facialis to the canalis cavernosus through the prootic (Figs. 11A, 11D). The position of 1061 the geniculate ganglion is inferred to be located in the canalis cavernosus, where it branches into 1062 the vidian and hyomandibular nerves (Figs. 11A, 11D). Whereas a sulcus for the hyomandibular 1063 nerve is visible in the wall of the canalis cavernosus in L. olivacea and Chelonia mydas, the 
1064 hyomandibular branch of the facial nerve has no osteological correlate in E. imbricata (Figs. 11A, 1065 11D), Caretta caretta, and N. depressus, and is thus inferred to be fully contained within the canalis 1066 cavernosus. The canalis pro ramo nervi vidiani is present in E. imbricata, Chelonia mydas, and N. 1067 depressus, formed by the pterygoid, and connects the canalis cavernosus to the canalis caroticus 1068 internus (Figs. 11A-11B, 11D). No canalis pro ramo nervi vidiani is visible in the specimens we 1069 used for L. olivacea and Caretta caretta and the path of the vidian nerve is therefore unclear in 1070 these two taxa. No canalis nervus vidianus is visible in any observed cheloniids. Siebenrock (1897) 1071 noted that the vidian nerve of Caretta caretta, Chelonia mydas, and E. imbricata is contained in 1072 the sulcus in which the internal carotid artery lies once it has joined the sulcus cavernosus, and 1073 Soliman (1964) clearly shows a vidian nerve in his illustrations of the cranial nerves in 1074 Eretmochelys imbricata. In the absence of separate canals for the vidian nerve, we infer that this 1075 nerve usually passes through the canalis caroticus internus and then through the sulcus cavernosus, but does not form an anterior vidian canal (Fig. 11D).

1077

\section{Dermochelyidae (Fig. 12)}

Canalis caroticus internus - The internal carotid artery enters the skull from posterior through the fenestra postotica. The actual foramen posterius canalis carotici interni is positioned deeply within the cavum acustico-jugulare and formed by the prootic and pterygoid. Unlike in most cryptodires, in which the foramen posterius canalis carotici interni and canalis caroticus internus are ventrally deeply embedded within the pterygoid bone, the internal carotid artery of Dermochelys coriacea (Figs. 12A-12C) seems to have a more dorsally positioned, superficial course with regard to the pterygoid. In particular, the foramen posterius canalis carotici interni and canalis caroticus internus are formed by raised ridges on the dorsal surface of the pterygoid, the 
1087 lateral of which is the comparatively shallow extension of the crista pterygoidei. The crista 1088 pterygoidei and the medial ridge are then roofed by the prootic to form the canalis caroticus 1089 internus. The canalis caroticus internus then continues between the pterygoid and prootic for a 1090 short distance, before the canal becomes encased by the basisphenoid and pterygoid more 1091 anteriorly. These observations differ from Albrecht (1976), who stated that the internal carotid 1092 artery is only surrounded by the pterygoid. As in cheloniids, the internal carotid artery joins the 1093 sulcus cavernosus and the split into the palatine and cerebral branches is not covered by bone 1094 (Nick, 1912; Albrecht, 1976; Evers \& Benson, 2019; Figs. 12A, 12D). No canalis caroticus 1095 lateralis, canalis caroticus basisphenoidalis, and canalis carotico-pharyngealis are present in our 1096 specimens. The absence of an ossified canalis caroticus basisphenoidalis in D. coriacea is unique

1097

1098

1099

1100

1101

1102

1103

1104

1105

1106

1107

1108

1109 among extant turtles, although it has been observed in at least one fossil turtle, Sandownia harrisi (Evers \& Joyce, 2020). In D. coriacea, osteological correlates for a short cerebral artery sulcus exist nonetheless. Directly anteromedial to the basicranial exit of the canalis caroticus internus, the lateral surface of the basisphenoid shows a weak but broad sulcus ventrally to the vestigially developed clinoid processes. This sulcus is interpreted as an incompletely ossified, anteriorly open canalis caroticus basisphenoidalis, and its broad size indicates that the cerebral artery is relatively large in diameter. Although the path for the cerebral artery was thus not fully enclosed by bone in the specimens we examined, Albrecht (1976) noticed on the specimen he examined that a canalis caroticus basisphenoidalis was present, supporting statements by Nick (1912). D. coriacea is known for its incomplete ossification pattern. For instance, much of the lateral side of the braincase, which, in other turtles, is ossified by a ventrally tall descending process of the parietal, remains cartilaginous in $D$. coriacea (Nick, 1912), and much of the hyoid skeleton remains entirely cartilaginous (Schumacher, 1973). Therefore, it is possible that completely ossified canalis 
1110 caroticus basisphenoidalis develop in particularly old and well ossified individuals, so that our

1111 observations are not necessarily in contrast to those of Albrecht (1976) or Nick (1912). The canalis

1112 stapedio-temporalis is about three times larger than the canalis caroticus internus in both specimens

1113 (Table 2).

1114 Mandibular artery - We are unaware of studies that clarify the source of the mandibular artery

1115 in D. coriacea, but also find no clear osteological correlates that would suggest origin from the 1116 stapedial artery versus one of the arteries of the internal carotid.

1117 Canalis cavernosus - The canalis cavernosus of Dermochelys coriacea (Figs. 12A-12D) is 1118 located lateral to the canalis caroticus internus and connects the subtemporal region of the skull 1119 with the cavum acustico-jugulare. The canalis cavernosus is formed by the pterygoid, quadrate, 1120 and prootic and is extremely short. The foramen cavernosum is positioned distinctly posterior to 1121 the position of the anteriorly unossified trigeminal foramen. A distinct sulcus cavernosus, as 1122 developed in most turtles anterior to the foramen cavernosum, is not developed in D. coriacea, but 1123 the course of the vena capitis lateralis is still inferred to extend posteriorly along the dorsal surface 1124 of the pterygoid based on the position of the foramen cavernosum.

1125 Facial nerve canal system - The facial nerve canal extends ventrolaterally from the fossa 1126 acustico-facialis through the prootic (Figs. 12A, 12D). The canalis nervus facialis exits the prootic 1127 in a position anterodorsal to the divergence point of the canalis caroticus internus and the canalis 1128 cavernosus. The geniculate ganglion is inferred to contact the canalis caroticus internus (Fig. 12D). 1129 The canalis pro ramo nervi vidiani is therefore absent and the vidian nerve directly enters the 1130 canalis caroticus internus (Fig. 12D). The hyomandibular nerve joins the canalis cavernosus via a 1131 very short canalis nervus hyomandibularis proximalis and then leaves no osteological correlate 1132 within the posterior section of the canalis cavernosus (Fig. 12A). No canalis nervus vidianus is 
1133 visible, but Nick (1912) observed the vidian nerve paralleling the course of the internal carotid

1134 artery and, once the internal carotid artery has joined the sulcus cavernosus and split into the

1135 cerebral and palatine branches, paralleling the course of the palatine artery (Fig. 12D). This

1136 provides evidence for our interpretations of the facial nerve system in Dermochelys coriacea.

1138 Platysternidae (Fig. 13)

1139 Canalis caroticus internus - The internal carotid artery enters the skull anterior to the level of 1140 the columella auris and through the fenestra postotica. A short, dorsally open sulcus in the 1141 pterygoid leads within the cavum acustico-jugulare to the foramen posterius canalis carotici interni

1142 (Fig. 13A), which is formed by the pterygoid. The canalis caroticus internus (Figs. 13A-13B) is 1143 formed by the prootic, pterygoid, and basisphenoid. The canalis pro ramo nervi vidiani enters the 1144 canalis caroticus internus about mid-length (Fig. 13B). The canalis caroticus lateralis is absent in 1145 our specimen, which confirms Albrecht's (1976) observations that a foramen anterius canalis 1146 carotici lateralis is absent. In contrast, Jamniczky \& Russell (2007) identified a canalis caroticus 1147 lateralis in their specimen. Following the description of the canal that Jamniczky \& Russell (2007) 1148 identify as the canalis caroticus lateralis, we conclude that it is the canalis nervus vidianus instead 1149 (see below). A canalis carotico-pharyngealis is absent. The canalis caroticus basisphenoidalis, 1150 herein defined once the internal carotid artery enters the basisphenoid, is mainly formed by the 1151 basisphenoid, with ventral contributions of the pterygoid (Figs. 13A-13B). The narrowly-spaced 1152 foramina anterius canalis carotici basisphenoidalis are formed by the basisphenoid (Figs. 13A1153 13B). The canalis stapedio-temporalis is three times larger than the canalis caroticus internus and 1154 canalis caroticus basisphenoidalis (Table 2). The relative proportions between the canalis stapedio- 
1155 temporalis and canalis caroticus basisphenoidalis corroborate the results of Jamniczky \& Russell 1156 (2007).

1157 Mandibular artery - The placement of the mandibular artery within the canalis cavernosus is 1158 suggested by a faint groove along its lateral side (Figs. 13A-13D), but we are unaware of 1159 dissections that unambiguously document this path. Evers and Benson (2019) reported the 1160 presence of a foramen arteriomandibulare. We are able to confirm the presence of symmetric 1161 openings between the canalis cavernosus and the temporal fossa in our specimen, the same as 1162 employed by Evers and Benson (2019), but note that the anterior wall of the canalis cavernosus is 1163 extremely thin and that these openings are elongate slits. The study of other specimens should 1164 clarify if these indeed represent the exit foramina of the mandibular artery or the incomplete 1165 ossification of the anterior wall of the canalis cavernosus in this particular specimen.

1166 Canalis cavernosus - The canalis cavernosus (Figs. 13A-13D) extends from the foramen 1167 cavernosum to the level of the foramen stapedio-temporale and is bordered by the quadrate, 1168 prootic, and pterygoid. The lateral wall of the canalis cavernosus is incompletely ossified in our 1169 specimen, exposing the canal along the lateral pterygoid-prootic-quadrate surfaces toward the 1170 subtemporal fossa. The foramen cavernosum is formed by the prootic and pterygoid.

1171 Facial nerve canal system - The canalis nervus facialis extends laterally from the fossa 1172 acustico-facialis to the canalis cavernosus through the prootic (Fig. 13A). The position of the 1173 geniculate ganglion is inferred to be within the canalis cavernosus, where it splits into the 1174 hyomandibular and vidian nerves (Figs. 13A, 13D). The hyomandibular nerve has no osteological 1175 correlate within the canalis cavernosus (Figs. 13A, 13D). The vidian nerve enters the canalis pro 1176 ramo nervi vidiani, extends ventrolaterally along the prootic-pterygoid suture, and joins the canalis 1177 caroticus internus at about its mid-length (Figs. 13B, 13D). Anteriorly, the vidian nerve exits the 
1178 canalis caroticus internus at the level of the foramen nervi trigemini just posterior to its

1179 transformation into the cerebral canal. The vidian nerve extends anteriorly through the pterygoid 1180 and leaves the skull on the lateral surface of the pterygoid, anteroventrally to the foramen nervi 1181 trigemini (Figs. 13A-13B, 13D). More anteriorly, ventral to the anterior end of the epipterygoid,

1182 the vidian nerve likely re-enters the skull on the lateral surface of the pterygoid, and extends 1183 anteriorly through the pterygoid and palatine (Figs. 13A-13D). At its anterior end, the canalis 1184 nervus vidianus merges with canals coming from ventral and then splits into two branches (Figs. 1185 13A-13B). The medial branch joins the dorsal surface of the palatine and the lateral one joins the 1186 foramen palatinum posterius. Jamniczky \& Russell (2007) identified the canalis nervus vidianus 1187 as being the canal for the palatine artery, but its location within the pterygoid, its exit towards the 1188 foramen palatinum posterius, the reported likely absence of the palatine artery in this taxon 1189 (Albrecht, 1976), the fact it does not join the sulcus cavernosus, and its correspondence with the 1190 vidian nerve canal of other testudinoids support our identification instead. The foramina anterius 1191 canalis nervi vidiani are formed by the pterygoid and palatine.

1193 Emydidae (Fig. 14)

1194 Canalis caroticus internus - The internal carotid artery enters the skull through the fenestra 1195 postotica in Emydoidea blandingii, Emys orbicularis, Deirochelys reticularia, Glyptemys 1196 insculpta, Graptemys geographica, Pseudemys floridana, and Terrapene ornata, but in a 1197 relatively more ventral position in Clemmys guttata, Glyptemys muhlenbergii, and Terrapene 1198 coahuila. The foramen posterius canalis carotici interni (Fig. 14A) is formed by the prootic, 1199 pterygoid, and basisphenoid in C. guttata, D. reticularia, Emydoidea blandingii, Gl. insculpta, 1200 and T. ornata, by the prootic and pterygoid in Emys orbicularis, Gr. geographica, Gl. 
1201 muhlenbergii, and T. coahuila, and by the pterygoid and basisphenoid in P. floridana. This

1202 differs from Siebenrock (1897) and McDowell (1961) who only identified the pterygoid and

1203 prootic as the bones forming the foramen posterius canalis carotici interni in these emydids. The

1204 canalis caroticus internus (Fig. 14B) is formed by the prootic, pterygoid, and basisphenoid in all

1205 observed emydids but $P$. floridana, in which the carotid artery extends along the pterygoid-

1206 basisphenoid suture for its entire length. According to Jamniczky \& Russell (2007), the canalis

1207 caroticus internus of Chrysemys picta and Emys orbicularis is formed by the basisphenoid and

1208 pterygoid bones only. Siebenrock (1897) also noted for Trachemys ornata that the foramen

1209 posterius canalis carotici interni is formed by the pterygoid and the canalis caroticus internus by

1210 the pterygoid and basisphenoid. The canalis pro ramo nervi vidiani (Figs. 14A-14B) joins the

1211 internal carotid canal in all emydids but Gl. muhlenbergii, in which the vidian nerve takes a

1212 short-cut by directly entering the canalis caroticus lateralis. In all observed emydids, the split of

1213 the canalis caroticus internus into the canalis caroticus lateralis and canalis caroticus

1214 basisphenoidalis occurs along the pterygoid-basisphenoid suture (Figs. 14A-14B). The palatine

1215 artery extends anteriorly through the canalis caroticus lateralis along the pterygoid-basisphenoid

1216 suture and joins the sulcus cavernosus slightly posteriorly to the anterior end of the rostrum

1217 basisphenoidale (Figs. 14A-14B). In Gr. geographica, the anterior portion of the palatine artery

1218 shifts laterally and extends through the pterygoid only. The palatine artery is exposed ventrally

1219 for a short distance in C. guttata, D. reticularia, Gl. muhlenbergii, T. coahuila, and T. ornata

1220 through an unnamed fenestra. Albrecht (1967) noted the presence of a canalis carotico-

1221 pharyngealis in Trachemys scripta. Here, we confirm the presence of one canalis carotico-

1222 pharyngealis in D. reticularia, Emydoidea blandingii, Emys orbicularis, Gl. insculpta, and $P$.

1223 floridana, and two canalis carotico-pharyngealis in Gr. geographica. In C. guttata, Gl. 
1224 muhlenbergii, T. coahuila, and T. ornata, no canalis carotico-pharyngealis is visible but this

1225 might be due to the ventral exposure of the palatine artery. The cerebral artery extends

1226 anteromedially through the basisphenoid and penetrates the sella turcica along widely-spaced

1227 foramina anterius canalis carotici basisphenoidalis (Fig. 14A). The canalis stapedio-temporalis is

1228 the largest canal and the canalis caroticus lateralis the smallest in all observed emydids, with the

1229 canalis stapedio-temporalis being about ten times larger (Table 2). However, some variation

1230 occurs regarding the size of the canalis caroticus basisphenoidalis in comparison with the canalis

1231 caroticus lateralis. For instance, the canalis caroticus basisphenoidalis of D. reticularia is ten

1232 times larger than the canalis caroticus lateralis, whereas the canalis caroticus basisphenoidalis

1233 and canalis caroticus lateralis of Gr. geographica have a similar size. The eventual split

1234 occurring between the canalis nervus vidianus and canalis caroticus lateralis is likely to change

1235 the size of the canalis caroticus lateralis anterior to this split, as in T. ornata or D. reticularia, in

1236 which the canalis caroticus lateralis becomes much smaller anterior to the separation with the

1237 canalis nervus vidianus. Our results differ from Jamniczky \& Russell (2007) who found equally

1238 sized canalis caroticus lateralis and canalis caroticus basisphenoidalis in Emys orbicularis.

1239 Mandibular artery - The presence of the mandibular artery can be inferred from a sulcus

1240 located along the lateral wall of the canalis cavernosus (Albrecht, 1967; Figs. 14A-14D).

1241 Canalis cavernosus - The morphology of the canalis cavernosus (Figs. 14A-14D) is very

1242 similar in all observed emydids. The canalis cavernosus extends from the foramen cavernosum to

1243 the level of the foramen stapedio-temporale and is formed by the quadrate, prootic and pterygoid.

1244 The foramen cavernosum is formed by the prootic and pterygoid.

1245 Facial nerve canal system - The facial nerve extends ventrolaterally from the fossa acustico-

1246 facialis to the canalis cavernosus through the prootic (Figs. 14A, 14D). The position of the 
1247 geniculate ganglion is inferred to be within the canalis cavernosus in all emydids, giving off the 1248 hyomandibular and vidian nerves (Figs. 14A, 14D). The hyomandibular nerve has no

1249 osteological correlate within the canalis cavernosus in Gl. insculpta (Figs. 14A, 14D), Gl.

1250 muhlenbergii, and P. floridana. However, in C. guttata, D. reticularia, Emydoidea blandingii,

1251 Emys orbicularis, Gr. geographica, T. coahuila, and T. ornata, the course of the hyomandibular

1252 nerve can be seen as a sulcus in the wall of the canalis cavernosus. The vidian nerve of all

1253 emydids but Gl. muhlenbergii extends through the canalis pro ramo nervi vidiani to join the

1254 canalis caroticus internus (Figs. 14A-14B, 14D), and is bordered by the prootic and pterygoid, 1255 with minor contributions of the basisphenoid in C. guttata, Emys orbicularis, Gl, insculpta, and 1256 P. floridana. This slightly differs from Shiino (1913), who did not mention the basisphenoid as 1257 forming parts of the canalis pro ramo nervi vidiani in C. guttata. In Gl. muhlenbergii, the vidian nerve does not join the canalis caroticus internus and extends through the prootic and pterygoid to merge anteriorly with the canalis caroticus lateralis. The vidian nerve is then inferred to follow the course of the canalis caroticus lateralis into the sulcus cavernosus. Anterior to the foramen anterius canalis caroticus lateralis, at the level of the anterior margin of the descending process of the parietal, an extremely short canal that might contain a portion of the vidian nerve crosses the pterygoid medio-laterally. In C. guttata, Emydoidea blandingii, and Emys orbicularis, the vidian nerve is inferred to pass through the canalis caroticus internus and canalis caroticus lateralis to join the sulcus cavernosus, and anteriorly pierces the pterygoid to enter the canalis nervus vidianus that is formed by the pterygoid and epipterygoid in C. guttata, by the pterygoid, epipterygoid, and parietal in Emydoidea blandingii, and Emys orbicularis. The foramen anterius canalis nervi vidiani is formed by the pterygoid and epipterygoid in C. guttata, by the pterygoid and parietal in Emydoidea blandingii, and by the epipterygoid, palatine, and parietal in Emys 
1270 orbicularis. D. reticularia exhibits a specific pattern on each side. On the left side, the vidian

1271 nerve splits from the canalis caroticus internus posterior to its split into the canalis caroticus

1272 lateralis and canalis caroticus basisphenoidalis, and the canalis nervus vidianus is formed by

1273 contributions of the prootic, pterygoid, basisphenoid, epipterygoid and parietal. On the right side,

1274 the vidian nerve is inferred to follow the course of the canalis caroticus internus and canalis

1275 caroticus lateralis, then splits from the canalis caroticus lateralis to extend through its own canal

1276 which is formed by the pterygoid, epipterygoid, and parietal. The foramina anterius canalis nervi

1277 vidiani are formed by the epipterygoid and parietal, and located ventrolaterally to the anterior

1278 margin of the processus inferior parietalis. In Gl. insculpta (Figs. 14A-14D), anterior to the

1279 canalis pro ramo nervi vidiani, the vidian nerve remains distinguishable from the canalis

1280 caroticus internus for a short distance as a hump located on the lateral side of the latter. The

1281 vidian nerve then splits from the canalis caroticus internus, extends anteriorly along the

1282 pterygoid-basisphenoid suture, and joins the posterior portion of the canalis caroticus lateralis.

1283 Posterior to the foramen anterius canalis carotici lateralis, the vidian nerve diverges from the

1284 canalis caroticus lateralis to extend through the pterygoid and exits the skull ventrolaterally to the

1285 anterior margin of the processus inferior parietalis. In Gr. geographica, P. floridana, T. coahuila

1286 and T. ornata, the vidian nerve is inferred to follow the course of the canalis caroticus internus

1287 and canalis caroticus lateralis, then splits from the latter posterior to or at the level of the foramen

1288 anterius canalis carotici lateralis, and extends anteriorly through the canalis nervus vidianus. The

1289 canalis nervus vidianus and foramen anterius canalis nervi vidiani are formed by the pterygoid

1290 and palatine in Gr. geographica, and by the pterygoid and epipterygoid in T. coahuila and $T$.

1291 ornata. The canalis nervus vidianus of $P$. floridana is formed by the pterygoid and palatine, but

1292 the foramen anterius canalis nervi vidiani by the palatine only. Siebenrock (1897) noted that the 
1293 canalis nervus vidianus of Trachemys ornata is formed by the pterygoid and palatine. Albrecht

1294 (1967) described a different pattern for the vidian nerve in Trachemys scripta, Chrysemys picta, 1295 and Pseudemys concinna. In these taxa, some branches of the vidian nerve enter a canal that

1296 Albrecht (1967) calls 'posterior canalis nervi vidiani' and that extends anterolaterally from the

1297 canalis pro ramo nervi vidiani close to the canalis carotico-pharyngeale. The other branches of

1298 the vidian nerve follow the course of the canalis caroticus internus and canalis caroticus lateralis.

1299 Some of these latter branches then join the branches of the 'posterior canalis nervi vidiani' via

1300 the canalis carotico-pharyngeale, and together, extend anteriorly through his 'anterior canalis

1301 nervi vidiani' through the pterygoid and palatine. The 'anterior canalis nervi vidiani' then opens

1302 into the foramen palatinum posterius.

1303

1304 Testudinidae (Fig. 15)

1305 Canalis caroticus internus - In all examined testudinids, the internal carotid artery enters the 1306 skull through the fenestra postotica. A sulcus for the internal carotid artery is present on the 1307 dorsal side of the pterygoid in all observed specimens but Aldabrachelys gigantea. The foramen 1308 posterius canalis carotici interni (Fig. 15A) is formed within the cavum acustico-jugulare by the 1309 prootic and pterygoid in Agrionemys horsfieldii, Al. gigantea, Gopherus agassizii, Gopherus 1310 polyphemus, Indotestudo elongata, Indotestudo forstenii, Malacochersus tornieri, and Testudo

1311 marginata, by the pterygoid in Gopherus flavomarginatus, by the prootic, pterygoid, and

1312 basisphenoid in Kinixys erosa, and by the quadrate, prootic, pterygoid, and basisphenoid in 1313 Psammobates tentorius. The canalis caroticus internus (Fig. 15B) is formed by the prootic, 1314 pterygoid, and basisphenoid in all observed testudinids but P. tentorius, in which the quadrate 1315 posterolaterally contributes to it. Jamniczky \& Russell (2007) did not mention the prootic as 
1316 contributing to the canalis caroticus internus of G. polyphemus and Ag. horsfieldii. The canalis

1317 caroticus internus contacts the canalis pro ramo nervi vidiani in Ag. horsfieldii Al. gigantea, G.

1318 agassizii, G. flavomarginatus, G. polyphemus, I. forstenii, M. tornieri, P. tentorius, but not in I.

1319 elongata, K. erosa, and T. marginata. The canalis caroticus lateralis and canalis carotico-

1320 pharyngealis are absent in all observed testudinids, with exception of a highly asymmetric

1321 specimen of Manouria impressa, which possess a canalis caroticus lateralis on the right side, but

1322 not on the left side. The canal on the right side appears to be a 'regular' canalis caroticus lateralis

1323 in the sense that it is in the expected position of such a canal in the basisphenoid-pterygoid

1324 suture, and that it extends toward an exiting foramen within the sulcus cavernosus. The canal

1325 size is similar to that in geoemydids. As this specimen appears to display a congenital

1326 abnormality, we disregard it from further consideration, but note that it may be informative in

1327 better understanding how the cranial circulation patterns originate during development. Our

1328 identification of the only canal that splits from the canalis caroticus internus as being the canalis

1329 nervus vidianus (see Figs. 15A-15B) rests upon three criteria that are encountered in all

1330 observed testudinids. First, this canal always extends anteriorly in a relative lateral position

1331 through the pterygoid. Second, the canal does not extend along the pterygoid-basisphenoid

1332 suture, unlike in all turtles with an unambiguous canalis caroticus lateralis. And third, the canal

1333 never connects to the sulcus cavernosus, whereas the canalis caroticus lateralis, when present in

1334 other turtles, connects to the sulcus cavernosus via the foramen anterius canalis caroticus lateralis

1335 along the pterygoid-basisphenoid suture. These criteria also imply that the palatine artery is

1336 likely absent in the taxa we observed. However, its presence has been highlighted in several

1337 studies. Shindo (1914) noted the presence of a very small palatine artery in Testudo graeca,

1338 splitting from the internal carotid artery close to the foramen anterius canalis carotici 
1339 basisphenoidalis, and extending anteriorly with the vidian nerve through the palatine. McDowell

1340 (1961) made a similar statement for the testudinids he dissected, observing a vestigial palatine

1341 artery in Gopherus berlandieri and T. graeca that extends through its own canal separate from

1342 the vidian nerve. Albrecht (1976) mentioned the palatine artery as being present but vestigial in

1343 G. flavomarginatus, G. polyphemus, M. tornieri, and Chelonoidis nigra, but these claims are not

1344 based on dissections and he did not provide detailed descriptions about where the palatine canal

1345 is located. Jamniczky \& Russell (2007) finally stated that the canalis caroticus lateralis and

1346 foramen anterius canalis carotici lateralis are absent in G. polyphemus. Several possibilities exist

1347 to explain these differences. First, it is possible that the presence of the palatine artery is highly

1348 polymorphic, but the systematic absence in our sample makes that somewhat unlikely. Second, it

1349 is possible that Shindo (1914) and McDowell (1961) misidentified the vidian nerve as the

1350 palatine artery and that the palatine artery is systematically absent. Third, it is possible that the

1351 palatine artery of tortoises regularly follows the course of the vidian nerve into the palatine, as

1352 hinted by Shindo (1914). Our criteria of homology may therefore have misled us to conclude the

1353 palatine artery to be systematically absent. Future studies using wet specimens will be needed to

1354 clarify this issue. The cerebral artery extends anteromedially through the basisphenoid in all

1355 testudinids to penetrate the sella turcica (Fig. 15A), with exception of G. flavomarginatus, where

1356 the artery extends along the pterygoid-basisphenoid suture before entering the basisphenoid. The

1357 foramina anterius canalis carotici basisphenoidalis are widely spaced (Fig. 15A). The canalis

1358 stapedio-temporalis is overall the largest canal and the canalis caroticus basisphenoidalis the

1359 smallest in all observed taxa (Table 2), which contradicts Jamniczky \& Russell (2007) who

1360 described the canalis stapedio-temporalis in G. polyphemus to be equally sized to the canalis

1361 caroticus internus and canalis caroticus basisphenoidalis. However, as in emydids, some 
1362 variation occurs. The canalis stapedio-temporalis of G. agassizii is more than ten times larger

1363 than the canalis caroticus basisphenoidalis, whereas the canalis stapedio-temporalis of I. forstenii

1364 is only slightly larger than the canalis caroticus basisphenoidalis. The canalis caroticus

1365 basisphenoidalis of $I$. forstenii is also slightly larger than the canalis caroticus internus, whereas

1366 the canalis caroticus basisphenoidalis is always smaller than the canalis caroticus internus in

1367 other observed testudinids.

1368 Mandibular artery - The mandibular artery of testudinoids splits off the stapedial artery

1369 within the cavum acustico-jugulare, passes through the canalis cavernosus, and exits the skull

1370 through the trigeminal foramen (McDowell, 1961; Albrecht, 1967; Figs. 15A-15D). The passage

1371 within the canalis cavernosus is typically visible as a lateral sulcus. McDowell (1961) noted,

1372 however, that the mandibular artery exits at an earlier point through a separate foramen that he

1373 named the foramen arteriomandibulare. The variable presence of this foramen was utilized by

1374 Crumly $(1982,1994)$ to infer phylogenetic relationships among tortoises. Here, we confirm the

1375 presence of the foramen in G. polyphemus and additionally report it for G. agassizii (Figs. 15A-

1376 15B), G. flavomarginatus, Al. gigantea, and K. erosa. This foramen is formed by the quadrate

1377 and pterygoid in Gopherus spp., by the quadrate in Al. gigantea, and by the quadrate, prootic,

1378 and pterygoid in K. erosa. The mandibular artery of M. tornieri (on the right side) and $T$.

1379 marginata is not contained in the canalis cavernosus for a short distance between the foramen

1380 stapedio-temporale and the foramen cavernosum and likely extends through its own canal. In $\mathrm{Ag}$.

1381 horsfieldii, I. forstenii, I. elongata, and P. tentorius, no separate mandibular artery foramen is

1382 evident, indicating that the arteria mandibularis passes anteriorly through the canalis cavernosus

1383 and then exits the skull through the trigeminal foramen. 
Canalis cavernosus - The canalis cavernosus (Figs. 15A-15D) extends from the foramen

1385

1386

1387

1388

1389

1390

1391

1392

1393

1394

1395

1396

1397

1398

1399

1400

1401

1402

1403

1404

1405

1406

cavernosum to the level of the foramen stapedio-temporale. In M. tornieri, the lateral wall of the canalis cavernosus is partially unossified, exposing it towards the subtemporal fossa. The canalis cavernosus of testudinids is otherwise formed by the quadrate, prootic, and pterygoid. The foramen cavernosum is formed by the prootic and pterygoid.

Facial nerve canal system - The facial nerve extends laterally through the prootic from the fossa acustico-facialis to the canalis cavernosus in Ag. horsfieldii, G. agassizii (Figs. 15A, 15D), G. flavomarginatus, G. polyphemus, K. erosa, M. tornieri, and T. marginata, and the geniculate ganglion is inferred to be within the canalis cavernosus. The geniculate ganglion is inferred to be located medially to the canalis cavernosus and dorsally to the canalis caroticus internus in $\mathrm{Al}$. gigantea, I. elongata, and I. forstenii. P. tentorius exhibits asymmetry, as the geniculate ganglion is located in between the canalis cavernosus and canalis caroticus internus on the right side, but contacts the canalis caroticus internus on the left side. The geniculate ganglion branches into the hyomandibular and vidian nerves. The hyomandibular nerve has no osteological correlate within the canalis cavernosus in G. flavomarginatus and I. elongata, but its course can be seen as a sulcus in the wall of the canalis cavernosus in Ag. horsfieldii, G. agassizii (Fig. 15A), I. forstenii, K. erosa, M. tornieri, P. tentorius, and T. marginata, and it extends through its own canalis nervus hyomandibularis distalis in Al. gigantea and G. polyphemus. In all observed testudinids but $K$. erosa, and T. marginata, the vidian nerve extends through the canalis pro ramo nervi vidiani to connect with the canalis caroticus internus, and leaves the latter more anteriorly to extend through the canalis nervus vidianus (Figs. 15A-15B, 15D). The canalis pro ramo nervi vidiani is formed by the prootic and pterygoid in Ag. horsfieldii, Al.gigantea, G. agassizii, G. flavomarginatus, G. polyphemus, I. elongata, and I. forstenii, but only by the prootic in $M$. 
1407

1408

1409

1410

1411

1412

1413

1414

1415

1416

1417

1418

1419

1420

1421

1422

1423

1424

1425

1426

1427

1428

1429

tornieri and P. tentorius. The vidian nerve of K. erosa and T. marginata extends ventromedially from the geniculate ganglion but does not join the canalis caroticus internus and instead directly enters the canalis nervus vidianus. Additional variation exists regarding the bones forming the canalis nervus vidianus and foramina anterius canalis nervi vidiani. The canalis nervus vidianus is formed by the prootic, pterygoid, and palatine in Ag. horsfieldii, G. polyphemus, and M. tornieri, by the pterygoid, epipterygoid, and palatine in Al. gigantea, by the pterygoid and palatine in G. agassizii, I. elongata, and P. tentorius, by the pterygoid, epipterygoid, palatine, and parietal in G. flavomarginatus, by the pterygoid in I. forstenii, by the prootic, pterygoid, palatine, and parietal in $K$. erosa, and by the prootic, pterygoid, epipterygoid, palatine, and parietal in T. marginata. In G. agassizii, the vidian nerve exits the skull anteroventrally to the foramen nervi trigemini for a short distance, before re-entering it by piercing the lateral surface of the palatine. The foramina anterius canalis nervi vidiani are formed by the pterygoid and palatine in Ag. horsfieldii, M. tornieri, and P. tentorius, by the epipterygoid and palatine in $A l$. gigantea, by the palatine in G. agassizii, G. polyphemus, and I. elongata, by the palatine and parietal in G. flavomarginatus and K. erosa, by the pterygoid in I. forstenii, and by the epipterygoid, palatine, and parietal in T. marginata. Gaupp (1888) noted that the canalis nervus vidianus of Testudo graeca is formed by the basisphenoid and pterygoid.

\section{Geoemydidae (Fig. 16)}

Canalis caroticus internus - In all observed geoemydids, the internal carotid artery enters the skull through the fenestra postotica. A sulcus for the internal carotid artery is formed on the dorsal side of the pterygoid. The foramen posterius canalis carotici interni (Fig. 16A) is located within the cavum acustico-jugulare and formed by the prootic and the pterygoid in all taxa in our 
1430 sample but Geoclemys hamiltonii, where it is only formed by the pterygoid. This slightly differs

1431 from Siebenrock (1897) and McDowell (1961) who only identified the pterygoid as forming the

1432 foramen posterius canalis carotici interni in Cyclemys dentata, Geoclemys hamiltonii, and

1433 Morenia sp. McDowell (1961) also noted that the opisthotic contributes to the foramen posterius

1434 canalis carotici interni of Batagur sp., which is not the case in the specimen of Batagur baska we

1435 studied. The canalis caroticus internus (Fig. 16B) is formed by the prootic, pterygoid, and

1436 basisphenoid in all observed geoemydids but Geoclemys hamiltonii, in which the canalis

1437 caroticus internus is formed by the pterygoid and basisphenoid only. Jamniczky \& Russell

1438 (2007) noted that the canalis caroticus internus of Cuora amboinensis and Rhinoclemmys

1439 pulcherrima is formed by the basisphenoid and pterygoid. The canalis pro ramo nervi vidiani

1440 enters the canalis caroticus internus in all geoemydids (as illustrated on Fig. 16A for B. baska)

1441 but Cyclemys dentata, Geoemyda spengleri, and Rhinoclemmys melanosterna. The canalis

1442 caroticus internus splits into the canalis caroticus lateralis and canalis caroticus basisphenoidalis

1443 in all sampled taxa (as illustrated on Figs. 16A-16B for B. baska) but Malayemys subtrijuga,

1444 Pangshura tecta, and R. melanosterna, where the lateral carotid canal appears to be absent.

1445 Jamniczky \& Russell (2007) noted the absence of the canalis caroticus lateralis and foramen

1446 anterius canalis carotici lateralis in $C u$. amboinensis and $R$. pulcherrima. The canalis caroticus

1447 lateralis extends anteriorly along the pterygoid-basisphenoid suture in B. baska (Figs. 16A-16B),

1448 Cu. amboinensis, Cy. dentata, Geoclemys hamiltonii, Geoemyda spengleri, Mauremys leprosa,

1449 and Mo. ocellata, and the foramen anterius canalis carotici lateralis is formed by the pterygoid

1450 and basisphenoid in these taxa. In S. crassicollis, the canalis caroticus lateralis and foramen

1451 anterius canalis carotici lateralis are formed by the pterygoid only. The canalis carotico-

1452 pharyngealis is absent in all observed specimens but $\mathrm{Cu}$. amboinensis, in which one canalis 
1453 carotico-pharyngealis is present. The canalis caroticus basisphenoidalis and foramen anterius

1454 canalis carotici basisphenoidalis are formed by the basisphenoid in all geoemydids (see Figs.

$145516 \mathrm{~A}-16 \mathrm{~B}$ for $B$. baska) but $S$. crassicollis, in which the pterygoid posteriorly contributes to the

1456 canalis caroticus basisphenoidalis. The foramina anterius canalis carotici basisphenoidalis are

1457 widely separated (Fig. 16A). In all observed geoemydids, the canalis stapedio-temporalis is the

1458 largest canal and the canalis caroticus lateralis, when present, the smallest (Table 2). As in

1459 emydids and testudinids, some variation regarding canal size occurs. The canalis stapedio-

1460 temporalis is generally nearly ten times larger than the canalis caroticus lateralis, if present, but

1461 this difference is more important in Cy. dentata and Geoemyda spengleri, in which the canalis

1462 caroticus lateralis is greatly reduced, and in Malayemys subtrijuga, P. tecta, and $R$.

1463 melanosterna, in which the canalis caroticus lateralis is absent. Moreover, the canalis stapedio-

1464 temporalis is about five times larger than the canalis caroticus basisphenoidalis in B. baska and

$1465 C u$. amboinensis, but only twice as large as the canalis caroticus basisphenoidalis in Malayemys

1466 subtrijuga and R. melanosterna, as noted by Jamniczky \& Russell (2007) in Rhinoclemmys

1467 pulcherrima.

1468 Mandibular artery - Siebenrock (1897) described a similar pattern for the mandibular artery

1469 of Cy. dentata as in testudinids, but this is not the case in our specimen. However, a distinct

1470 protrusion is visible on the lateral wall of the canalis cavernosus of this specimen that likely

1471 corresponds to the mandibular artery (Figs. 16A-16D).

1472 Canalis cavernosus - The canalis cavernosus (Figs. 16A-16D) extends from the foramen

1473 cavernosum to the level of the foramen stapedio-temporale and is formed by the quadrate and

1474 prootic. The foramen cavernosum is formed by the prootic and pterygoid. 
Facial nerve canal system - The canalis nervus facialis extends laterally through the prootic

1476 from the fossa acustico-facialis to the canalis cavernosus (Fig. 16A). The position of the

1477 geniculate ganglion is inferred to be within the canalis cavernosus in all observed geoemydids

1478 (see Figs. 16A, 16D for B. baska) but Mauremys leprosa, in which the geniculate ganglion is not

1479 in contact with the canalis cavernosus, and R. melanosterna, in which the geniculate ganglion

1480 contacts the canalis caroticus internus. The geniculate ganglion splits into the hyomandibular and

1481 vidian nerves (Figs. 16A, 16D). The course of the hyomandibular nerve is visible as a sulcus in

1482 all observed geoemydids (Figs. 16A, 16D) but Mo. ocellata, in which the hyomandibular nerve

1483 has no osteological correlate within the canalis cavernosus and is inferred to follow the course of

1484 it. In all geoemydids but $C y$. dentata, Geoemyda spengleri, and $R$. melanosterna, the vidian

1485 nerve extends through the canalis pro ramo nervi vidiani to join the canalis caroticus internus

1486 (Figs. 16A, 16D). Three patterns for the vidian nerve can be identified for the taxa exhibiting a

1487 canalis pro ramo nervi vidiani. In Malayemys subtrijuga and P. tecta, the vidian nerve leaves the

1488 canalis caroticus internus anterior to the canalis pro ramo nervi vidiani to enter the canalis nervus

1489 vidianus. In Mauremys leprosa and Mo. ocellata, the vidian nerve leaves the canalis caroticus

1490 internus, and anteriorly enters the canalis caroticus lateralis to extend into the sulcus cavernosus,

1491 and then leaves the latter laterally, anterior to the foramen anterius canalis carotici lateralis. In $B$.

1492 baska (Figs. 16A, 16D), Cu. amboinensis, Geoclemys hamiltonii, and S. crassicollis, the vidian

1493 nerve is inferred to extend through the canalis caroticus internus and canalis caroticus lateralis

1494 into the sulcus cavernosus, and then enters the canalis nervus vidianus anterior to the foramen

1495 anterius canalis carotici lateralis. The vidian nerve of Geoclemys hamiltonii is laterally exposed

1496 for a short distance. Cy. dentata and Geoemyda spengleri diverge from these patterns by having

1497 a vidian nerve that respectively merges with one and two canals originating from the posterior 
1498 portion of the canalis caroticus internus. The vidian nerve of Geoemyda spengleri also shortly

1499 extends through the canalis caroticus lateralis. As the facial nerve system of $R$. melanosterna

1500 connects to the canalis caroticus internus, the canalis pro ramo nervi vidiani is absent and the

1501 vidian nerve directly joins the canalis caroticus internus, follows the course for the internal

1502 carotid artery for a short distance, and enters the canalis nervus vidianus. As in testudinids, a

1503 substantial amount of variation exists about the bones forming the canalis nervus vidianus and

1504 foramina anterius canalis nervi vidiani. The canalis nervus vidianus is formed by the pterygoid

1505 and palatine in B. baska and P. tecta, by the pterygoid, palatine, and parietal in Cu. amboinensis,

1506 Geoclemys hamiltonii, Malayemys subtrijuga, Mo. ocellata, and S. crassicollis, by the prootic,

1507 pterygoid, and parietal in Cy. dentata, by the prootic and pterygoid in Geoemyda spengleri, by

1508 the pterygoid, epipterygoid, and parietal in Mauremys leprosa, and by the pterygoid,

1509 epipterygoid, palatine, and parietal in R. melanosterna. The foramina anterius canalis nervi

1510 vidiani are formed by the palatine in B. baska, Mo. ocellata, P. tecta, and R. melanosterna, by

1511 the pterygoid and parietal in Cu. amboinensis, by the parietal in Cy. dentata and Mauremys

1512 leprosa, by the palatine and parietal in Geoclemys hamiltonii, Malayemys subtrijuga, and $S$.

1513 crassicollis, and by the pterygoid in Geoemyda spengleri.

\section{DISCUSSION}

1516 Summary of carotid canal system

1517 Internal carotid artery - Almost all extant turtles have an internal carotid artery that is fully

1518 enclosed in a canalis caroticus internus that extends anteriorly into the basicranium. The sole

1519 exception is podocnemidids, where the internal carotid artery passes through a large opening, the

1520 cavum pterygoidei, which is unique to that clade. The highly derived placement of 
1521 podocnemidids within Testudines strongly suggests that this is a secondary modification. We

1522 note here, that it is a matter of semantic preference if the foramen posterius canalis carotici

1523 interni is thought to be present in podocnemidids, but modified (e.g., sensu Gaffney, Tong \&

1524 Meylan, 2006), or absent due to the presence of a cavum pterygoidei (as defined herein). Our

1525 study therefore does not provide novel insights that contradict previous ones. The pterygoid bone

1526 is always involved in the formation of the foramen posterius canalis carotici interni in

1527 cryptodires but never contributes to this foramen in extant pleurodires (see Table 3 for a

1528 summary of the main differences between internal carotid artery systems). This generalization, of

1529 course, only applies in the strict sense of the foramen posterius canalis carotici interni employed

1530 herein, as the enlarged cavum pterygoidei of podocnemidis has a clear contribution from the

1531 pterygoid and has been interpreted as the foramen posterius canalis carotici interni by others

1532 (e.g., Siebenrock, 1897). Incidentally, a contribution from the pterygoid is apparent in the stem

1533 pleurodire Notoemys laticentralis (Lapparent de Broin, de la Fuente \& Fernandez, 2007). This

1534 may suggest that a pterygoid contribution is plesiomorphic for crown Testudines.

1535 Cerebral artery - Little variation exists for the cerebral artery in extant turtles outside of

1536 Chelonioidea. All examined taxa have a canalis caroticus basisphenoidalis that extends through

1537 the basisphenoid and exits in the sella turcica. The only exception to this is found in

1538 Dermochelys coriacea, in which the cerebral artery might not be fully enclosed by bone, a

1539 feature that has otherwise only been reported in the fossil turtle Sandownia harrisi (Evers \&

1540 Joyce, 2020). The exiting foramina for the cerebral artery, the foramina anterius canalis carotici

1541 basisphenoidalis, are paired across the skull midline in nearly all species of extant turtles. Only

1542 in three species of cheloniids (Lepidochelys olivacea, Caretta caretta, Natator depressus), the

1543 left and right canalis caroticus basisphenoidalis merge within the basisphenoid and exit the sella 
1544 turcica via a single, median foramen (see also Hooks, 1998; Evers, Barrett \& Benson, 2019 for

1545 fossil occurrences of this feature). Otherwise, variation regarding the cerebral circulation is

1546 limited to the spacing of the foramina anterius canalis carotici basisphenoidalis (Hirayama,

1547 1998). In our sample, the foramina anterius canalis carotici basisphenoidalis are widely-spaced in

1548 all turtles but Macrochelys temminckii, platysternids, and the remaining chelonioids, in which

1549 these openings are rather narrowly-spaced.

1550 The repeated loss of the lateral carotid canal - We observe the complete loss of the lateral

1551 carotid canal in all pleurodires (contra Albrecht, 1967, 1976; Gaffney, Tong \& Meylan, 2006;

1552 Hermanson et al., 2020), carettochelyids (see also Joyce, Volpato \& Rollot, 2018), platysternids,

1553 testudinids, and some geoemydids, in all cases due to the absence of a palatine artery. A canalis

1554 caroticus lateralis is furthermore absent in chelonioids, but not a result of the absence of the

1555 palatine artery, but due to its anterior displacement (see also Zangerl, 1953; Albrecht, 1976;

1556 Gaffney, 1979; Evers \& Benson, 2019). As these conclusions are perhaps the most novel

1557 conclusion of this study, we briefly repeat out rationales, while outlining difficulties for

1558 paleontologists assessing fossil material.

1559 The special embedding of the internal carotid artery within the cavum pterygoidei in

1560 podocnemidids makes it difficult to evaluate the possible presence of a lateral carotid canal in

1561 this group, as no actual canal of the size of a blood vessel is developed between the cavum

1562 pterygoidei and sulcus cavernosus. Previous authors, such as Gaffney, Tong \& Meylan (2006) or

1563 Hermanson et al. (2020), interpreted the fenestra between the cavum pterygoidei and sulcus

1564 cavernosus to be a trough for the palatine artery. By contrast, we hypothesize the universal

1565 absence of a palatine artery in podocnemidids based on the dissection study of Albrecht (1976),

1566 who only found a vestigial blood vessel to pass this fenestra in one out of six specimens. The 
1567 podocnemidid "foramen anterius canalis carotici lateralis" of previous authors is therefore an

1568 unnamed fenestra of unclear function. Our re-interpretation, incidentally, is more parsimonious

1569 than the interpretation of Hermanson et al. (2020), who inferred a loss of the palatine artery at

1570 the base of Pelomedusoides, its reappearance in Podocnemidoidae, and yet another loss for

1571 stereogenyine podocnemidids. Our model suggests the persistent absence of a palatine artery in

1572 all pelomedusoids, indeed, all pleurodires.

1573 The interpretation of chelids based on osteological material is difficult as well. Albrecht

1574 (1976) concluded that a lateral carotid canal is present in all chelids but Chelus fimbriatus, but

1575 noted at the same time that this canal does not hold a palatine artery, but rather the vidian nerve.

1576 If terminology is to reflect homology, chelids possess a vidian canal, not a lateral carotid canal.

1577 Although the said canal subtly reveals its identity as pertaining to the vidian nerve by connecting

1578 the geniculate ganglion to the sulcus cavernosus, it suspiciously resembles the lateral carotid

1579 canal of other turtles by approximating the pterygoid-basisphenoid suture. It is therefore

1580 understandable that this unusually located vidian canal has been misidentified as a lateral carotid

1581 canal in the literature (e.g., Hermanson et al., 2020; figured for Hydromedusa tectifera). As a

1582 result, the absence of the lateral carotid canal, which is ultimately based on the loss of the

1583 palatine artery, is a likely synapomorphy of Pleurodira.

1584 Within Trionychia, we infer the independent loss of the palatine artery for carettochelyids (see

1585 also Joyce, Volpato \& Rollot, 2018). Interestingly, the artery that extends through the short

1586 canalis caroticus lateralis in trionychids does not serve the function of a 'regular' palatine artery,

1587 but instead supplies the mandible (Albrecht, 1967). Thus, the lateral carotid canal of trionychids,

1588 although present according to our observations, fulfills a different role and complicates 
1589

1590

1591

1592

1593

1594

1595

1596

1597

1598

1599

1600

1601

1602

1603

1604

1605

1606

1607

1608

1609

1610

1611

assessment on its homology. We provide our reasoning for concluding that the mandibular artery

of trionychids is a repurposed palatine artery below (see discussion of mandibular artery).

In addition to the definite reductions in pleurodires and carettochelyids, the canalis caroticus

lateralis is lost several times within testudinoids, which generally show the largest amount of

variation. As the canalis caroticus lateralis is absent in Platysternon megacephalum, but present

in all emydids, there seems to have been one independent loss within Emysternia. Within

geoemydids, the canalis caroticus lateralis is generally present, with exception of Malayemys

subtrijuga, Pangshura tecta, and Rhinoclemmys melanosterna, which represent several

independent losses of the palatine artery based on current geoemydid in-group relationships (e.g.,

Garbin, Ascarrunz \& Joyce, 2018). The canalis caroticus lateralis is absent in all testudinids we

examined, with the exception of a highly unusual specimen of Manouria impressa (SMF 69777;

Fig. 17), which has an asymmetrical arterial pattern with a 'regular' canalis caroticus lateralis on

the left side, but none on the right side. The occurrence of the palatine artery had been mentioned by Shindo (1914), McDowell (1961), and Albrecht (1976) for some testudinids. Thus, although

the vast majority of testudinid specimens examined herein show no evidence for the presence of the palatine artery, it is possible that this feature is highly polymorphic within testudinids. To address this further, studies focusing on intraspecific variation regarding the palatine artery are necessary, which is beyond the scope of this contribution. We note, however, that the canalis caroticus lateralis is extremely small in diameter whenever it is present (Table 2). In summary, a highly reduced to absent palatine artery appears to be a common feature of all extant testudinoids.

Although the palatine artery is present in extant chelonioids (Albrecht, 1976), the internal carotid artery of these turtles enters the sulcus cavernosus prior to its split into the palatine and 
1612 cerebral branches, so that the palatine artery is never encased in a bony canal (Zangerl, 1953;

1613 Albrecht, 1976; Gaffney, 1979; Evers \& Benson, 2019). However, the condition likely evolved

1614 independently in dermochelyids and extant cheloniids, as stem-group cheloniids show a regular

1615 bifurcation pattern with a regularly enclosed palatine artery (Evers, Barrett \& Benson, 2019).

1616 The mandibular artery - The mandible of turtles is typically supplied by a large artery that

1617 variously originates from different parts of the carotid arterial system (Albrecht, 1967, 1976).

1618 These different origins make it difficult to homologize arteries, but the term 'mandibular artery'

1619 has historically been used for all variants of mandible-supplying arteries (e.g., Gaffney, 1979)

1620 and we generally adhere to this practice. However, as the mandible is sometimes supplied by two

1621 arteries from two sources, we here follow the convention of Albrecht $(1967,1976)$ by

1622 distinguishing between an anterior and posterior mandibular artery. The mandibular artery is

1623 mostly supplied by the stapedial artery in pleurodires, carettochelyids, chelydrids, and

1624 testudinoids, by the palatine artery in kinosternids, but by both sources in cheloniids (McDowell,

1625 1961; Albrecht, 1967, 1976). In trionychids, the mandibular artery takes the same proximal

1626 course as the palatine artery of other turtles (i.e., via the lateral carotid canal), but then only

1627 supplies the mandible and does not send a branch anteriorly to supply the facial region (Albrecht,

1628 1967). This raises the question of whether the 'mandibular artery' of trionychids is a modified

1629 palatine artery (in which case the palatine artery is present in trionychids), or neomorphic (in

1630 which case the palatine artery of trionychids was lost). This distinction is important for the

1631 reconstruction of the plesiomorphic condition for both crown Cryptodira and crown Testudines.

1632 Currently, unpublished CT scans of stem trionychians available to us (e.g., Basilemys sp.;

1633 Adocus sp.) indicate that a 'regular' palatine artery was present in stem-trionychians. This

1634 suggests that the mandibular artery of trionychids indeed is a palatine artery in which the 
1635 function of the artery (as in the skull region it supplies with blood) has changed. A similar

1636 change has occurred at least a second time, within kinosternids, but in that clade the palatine

1637 artery retains its 'regular' function and only adds the role of supplying the mandible (Albrecht, 1638 1967).

1639 In turtles in which the mandibular artery branches off the stapedial artery, this branching point 1640 can occur either before the stapedial artery enters the canalis stapedio-temporalis (as reported for 1641 cryptodires in which the mandible is supplied by a branch of the stapedial artery: Albrecht, 1967;

1642 Gaffney, 1979), or after it has left the foramen stapedio-temporale (as reported for pleurodires:

1643 Albrecht, 1967, 1976). Among pleurodires, Albrecht (1976) observed that the mandibular artery

1644 branches off the stapedial artery and identified two different patterns. In pelomedusids and 1645 chelids, with the exception of Chelodina longicollis, a large mandibular artery splits from the 1646 stapedial artery after the later has exited the skull through the foramen stapedio-temporale. In 1647 Chelodina longicollis and podocnemidids, the stapedial artery has two branches, the external and 1648 internal mandibular arteries. The external mandibular artery splits from the stapedial artery 1649 posterior to the cranium and extends anteroventrally to supply the tissue directly medial to the 1650 mandible. After exiting the skull through the foramen stapedio-temporale, the stapedial artery 1651 arches anteriorly over the fenestra subtemporalis and the internal mandibular artery splits from 1652 the latter lateral to the foramen nervi trigemini, and courses ventrally to the fossa Meckelii in the 1653 mandible. As either course bypasses bone, we are unable to find osteological correlates that 1654 would document this pattern in fossil taxa.

1655 When the mandibular artery branches off the stapedial artery within the cavum acustico1656 jugulare (testudinoids, chelydrids, carettochelyids, the posterior (vestigial) mandibular artery of 1657 chelonioids, and, potentially, dermatemydids), it takes an anteriorly directed course through the 
1658 canalis cavernosus. From there, it usually exits the cranium, either through the trigeminal

1659 foramen or a separate foramen arteriomandibulare. The latter exit has been observed directly

1660 from dissections for the posterior (vestigial) mandibular artery of the chelonioid Chelonia mydas

1661 (Albrecht, 1976) and the mandibular artery of the testudinids Gopherus berlandieri,

1662 Stigmochelys pardalis, and Chelonoidis denticulatus (McDowell, 1961).

1663 The presence of the foramen arteriomandibulare should serve as an excellent osteological 1664 correlate for the location of the mandibular artery in the canalis cavernosus. We confirm the 1665 previously established presence of this foramen in testudinids (McDowell, 1961), but note that 1666 Bramble (1971) and Crumly $(1982,1994)$ found additional variation, which may have relevance 1667 for systematics. Among testudinoids, Evers \& Benson (2019) reported a foramen 1668 arteriomandibulare for Platysternon megacephalum, but we here note by reference to the same 1669 specimen, that it is unclear if the slit-like foramina indeed represent a true foramen 1670 arteriomandibulare or the incomplete ossification to the anterior wall of the canalis cavernosus. 1671 In contrast, we here confirm the exit of the mandibular artery through a separate foramen in 1672 Carettochelys insculpta through the presence of a foramen arteriomandibulare in osteological 1673 material, as previously reported by Evers \& Benson (2019), in combination with personal 1674 observations of a stained specimen. This clearly contradicts the speculations of Joyce, Volpato \& 1675 Rollot (2018) that the mandibular artery of carettochelyids may be supplied by the certebral 1676 artery.

1677 Evers \& Benson (2019) also reported the presence of a foramen arteriomandibulare in the 1678 pelomedusid Pelomedusa subrufa. If correct, this observation would be particularly relevant, as 1679 it would indicate a completely different course of the mandibular artery (i.e. through the canalis 1680 cavernosus) than usually reported for pleurodires (Albrecht, 1976). Given that dissections by 
1681 Albrecht (1976) of Pelomedusa subrufa show that the canalis cavernosus houses no artery

1682 (contradicting observations by Evers \& Benson, 2019), we re-examined the specimen used by

1683 Evers \& Benson (2019: SMF 70504). The 'mandibular artery foramen' of that specimen is

1684 highly irregular around its margins, and parallels the anterior portion of the canalis cavernosus,

1685 much as in the specimen of Platysternon megacephalum mentioned above. We, therefore, here

1686 re-interpret the 'foramina' as the incompletely ossified anterior wall of the canalis cavernosus.

1687 Although the presence of a foramen arteriomandibulare is the best direct osteological

1688 correlate for the course of the mandibular artery, our segmentations show that the course of this

1689 artery through the canalis cavernosus is often indicated by a subtle subdivision of the latter,

1690 which can serve as a second, more subtle osteological correlate for the placement of the

1691 mandibular artery within the canalis cavernosus. Such a subdivision is present in Carettochelys

1692 insculpta (Fig. 6), Dermatemys mawii (Fig. 9), chelydrids (Fig. 10), cheloniids (Fig. 11),

1693 Platysternon megacephalum (Fig. 13), emydids (Fig. 14), testudinids (Fig. 15), and geoemydids

1694 (Fig. 16). In these clades the dorsolateral part of the canalis cavernosus is separated from the

1695 medioventral part by a longitudinal, dorsal constriction between these subsections of the canal,

1696 which is visible on models of the canalis cavernosus as a longitudinal sulcus. In coronal slices of

1697 CT scans of these taxa, the canalis cavernosus shows a reniform cross-section. This morphology

1698 is developed to different degrees. For instance, it is particularly strong in testudinids (Fig. 15)

1699 and geoemydids (Fig. 16). However, there is also within-clade variation to the feature. For

1700 instance, the subdivision of the canalis cavernosus is relatively subtle in the geoemydid

1701 Geoclemys hamiltonii, but well-developed to the point that almost two canals are developed in

1702 sections of the skull in the geoemydids Cyclemys dentata and Pangshura tecta. We suggest the

1703 presence of this subdivision within the canalis cavernosus to be an osteological correlate for the 
1704 condition that a mandibular artery extends through the canalis cavernosus. The slight spatial

1705 separation within the canalis cavernosus thereby represents the adjacent pathways for the vena

1706 capitis lateralis and mandibular artery. Two reasons for hypothesizing the subdivision as an

1707 indicator for the mandibular artery can be identified. The presence of the subdivision of the

1708 canalis cavernosus coincides with the passage of the mandibular artery through that structure in

1709 all taxa for which direct dissection observations have been made. Additionally, the foramen

1710 arteriomandibulare, i.e. a clear indication that the mandibular artery passes through the canalis

1711 cavernosus, is only ever present in taxa which also have the subdivision. Thus, the presence of

1712 this subdivision provides important information on some clades for which currently no dissection

1713 study is available, such as Dermatemys mawii. Thus, we speculate that the subdivision of the

1714 canalis cavernosus can be useful in the future to investigate the course of the mandibular artery

1715 in fossil clades.

1716 In summary, several patterns for the mandibular artery can be identified from the information

1717 available and summarized above: (i) the mandibular artery branches off the stapedial artery

1718 without extending through the canalis cavernosus in pleurodires, and the chelid Chelodina

1719 longicollis and podocnemidids have a second mandibular artery branch additionally supplying

1720 the mandible. In the second pattern (ii), the mandibular artery branches off the stapedial artery

1721 within the cavum acustico-jugulare, follows the course of the canalis cavernosus, and exits the

1722 skull through the foramen nervi trigemini or a separate foramen arteriomandibulare. This pattern

1723 is observed among cryptodires with the exceptions of kinosternids and trionychids. In

1724 kinosternids (iii), the mandibular artery does not branch off the stapedial artery but rather from

1725 the palatine artery, anterior to the foramen anterius canalis carotici lateralis. Finally (iv), the 
1726 mandibular artery of trionychids is interpreted to be a modified palatine artery and extends

1727 through the canalis caroticus lateralis.

1728

\section{The facial nerve system of turtles}

1730 Gaffney (1979) proposed two main patterns for the course of the facial nerve in pleurodires

1731 and cryptodires, respectively. According to this model, pleurodires have the geniculate ganglion

1732 located at the junction between the canalis nervus facialis and the canalis caroticus internus,

1733 whereas the geniculate ganglion of cryptodires is developed at the point of contact between the

1734 canalis nervus facialis and the canalis cavernosus. This hypothesis was extrapolated from data

1735 collected by Siebenrock (1897) and Soliman (1964) on a few species of cryptodires and

1736 pleurodires. The summary provided by Gaffney (1979) lead to the construction of a phylogenetic

1737 character for the passage of the hyomandibular branch of the facial nerve (e.g., Gaffney, Meylan

1738 \& Wyss, 1991). Since, few studies have further scrutinized the proposals of Gaffney (1979) (but

1739 see Evers \& Benson, 2019). Here, we propose that two main patterns of the facial nerve system

1740 can be recognized among extant turtles (Fig. 18). These patterns differ from one another in the

1741 relative position of the geniculate ganglion, but, unlike in Gaffney (1979), they do not

1742 correspond closely to the phylogenetic distinction of pleurodires and cryptodires (see Table 4 for

1743 a summary of the main differences between facial nerve systems).

1744 Our facial pattern I is characterized by a geniculate ganglion positioned within the canalis

1745 cavernosus, or at the interface between the canalis nervus facialis and the canalis cavernosus, and

1746 thus outside of the prootic (Fig. 18). Facial pattern I essentially corresponds to the 'cryptodiran

1747 pattern' of Gaffney (1979). However, facial pattern I is actually only realized in a subset of

1748 cryptodires, namely in chelydroids (kinosternids, chelydrids, Dermatemys mawii), cheloniids, 
1749 emysternians (emydids, Platysternon megacephalum), a subset of trionychids (Apalone spinifera,

1750 Apalone mutica, Amyda cartilaginea, and Pelodiscus sinensis among our sample), a subset of

1751 testudinids (Agrionemys horsfieldii, Gopherus agassizii, Gopherus flavomarginatus, Gopherus

1752 polyphemus, Kinixys erosa, Malacochersus tornieri, and Testudo marginata among our sample),

1753 and a subset of geoemydids (Batagur baska, Cuora amboinensis, Cyclemys dentata, Geoclemys

1754 hamiltonii, Geoemyda spengleri, Malayemys subtrijuga, Morenia ocellata, Pangshura tecta, and

1755 Siebenrockiella crassicollis among our sample). Our facial pattern I is further subdivided into

1756 three sub-patterns that are distinct in the way the proximal portion of the vidian nerve is

1757 transmitted anteriorly. Most turtles with facial pattern I have a canalis pro ramo nervi vidiani

1758 which transmits the vidian nerve from within the canalis cavernosus into the canalis caroticus

1759 internus, and we designate this condition as facial pattern IA. The facial pattern we name IB is

1760 similar in that a canalis pro ramo nervi vidiani is also present, but this canal extends to the

1761 canalis caroticus lateralis instead. Thus, the canalis pro ramo nervi vidiani intersects with the

1762 carotid canal system slightly more anteriorly than in facial pattern IA. Facial pattern IB is seen in

1763 the emydid Glyptemys muhlenbergii and the geoemydids Cyclemys dentata and Geoemyda

1764 spengleri. We observed a third pattern, named facial pattern IC, in the testudinids Kinixys erosa

1765 and Testudo marginata. These turtles lack a by-passage of the vidian nerve through the canalis

1766 caroticus internus or any other canal associated with the carotid arterial system altogether.

1767 Instead, the vidian nerve passes directly into the canalis nervus vidianus from the canalis

1768 cavernosus.

1769 Our facial pattern II is characterized by a geniculate ganglion position outside the canalis

1770 cavernosus and in a more proximal position with regard to the facial nerve stem coming from the

1771 brain, and thus within the prootic. All turtles with facial pattern II have a canalis nervus 
1772 hyomandibularis proximalis, which transmits the hyomandibular nerve from within the prootic to

1773 the canalis cavernosus. However, facial pattern II can be subdivided according to the course of

1774 the vidian nerve. In turtles with the facial pattern we name IIA, the vidian nerve is contained in a

1775 separate canal, the canalis pro ramo nervi vidiani, which extends from the geniculate ganglion to

1776 the canalis caroticus internus. The canalis pro ramo nervi vidiani in turtles of facial pattern IIA

1777 always extends through the prootic, and thus differs slightly from the canal with the same name

1778 in turtles of facial patterns IA and IB, in which the canal diverges from the canalis cavernosus

1779 and thus often from within the pterygoid. We retain the same name for all of these canals, as

1780 either transmit the vidian nerve toward the carotid arterial system. In facial pattern IIB, the

1781 vidian nerve directly enters the canalis caroticus internus and a canalis pro ramo nervi vidiani is

1782 absent. The direct transmission of the vidian nerve is enabled by an intersection of the canalis

1783 caroticus internus with the canalis nervus facialis. Facial patterns IIB and IIC share the direct

1784 intersection of the canalis nervus facialis and the canalis caroticus internus. However, in facial

1785 pattern IIC, a separate, anteriorly directed canalis nervus vidianus emerges from the area of the

1786 canal intersection. Thus, instead of passing through the carotid canal system, the vidian nerve

1787 directly enters its own canal, similar to facial pattern IC. Facial pattern IIA is found in

1788 podocnemidids, Carettochelys insculpta, some trionychids (Chitra indica, Cyclanorbis

1789 senegalensis, Cycloderma frenatum and Lissemys punctata among our sample), some testudinids

1790 (Aldabrachelys gigantea, Indotestudo elongata, and Indotestudo forstenii among our sample),

1791 and the geoemydid Mauremys leprosa. Facial pattern IIB, which essentially is the 'pleurodiran

1792 pattern' of Gaffney (1979), is found in most chelids and Pelomedusa subrufa, but additionally

1793 also in the cryptodires Dermochelys coriacea and Rhinoclemmys melanosterna. Facial pattern

1794 IIC is found in the chelid Chelus fimbriatus and the pelomedusid Pelusios subniger. It is 
1795

1796

1797

1798

1799

1800

1801

1802

1803

1804

1805

1806

1807

1808

1809

1810

1811

1812

1813

1814

1815

1816

noteworthy that our facial pattern IIA is basically identical to the facial nerve pattern recognized by Evers \& Benson (2019: see character 127.1) for thalassochelydians, which were found to be crownward stem-turtles in that study. Our finding of similar facial nerve patterns in some pleurodires (podocnemidids) and some of the phylogenetically earliest branching cryptodires (some trionychids and Carettochelys insculpta) possibly suggests, that facial pattern IIA could be plesiomorphic for crown-group turtles. However, only the re-examination of a broad set of fossil turtles can test this further.

Two additional sources of variation to the facial nerve system are apparent, but these are unrelated to the facial patterns outlined above. The first kind of variation relates to the posterior (=distal) path of the hyomandibular nerve. In all turtles, the posterior part of the hyomandibular nerve traverses or parallels the canalis cavernosus to reach the cavum acustico-jugulare.

However, the posterior course of the nerve may be situated (a) in a separate canal, the canalis nervus hyomandibularis distalis (hyomandibular pattern I); (b) a sulcus in the wall of the canalis cavernosus (hyomandibular pattern II); or (c) entirely within the canalis cavernosus (hyomandibular pattern III) (Fig. 19). This variation is independent of the presence of a canalis nervus hyomandibularis proximalis in turtles with facial pattern II, as the canalis nervus hyomandibularis proximalis of facial pattern II only transmits the proximal part of the hyomandibular nerve toward the canalis cavernosus. This is exemplified by turtles with facial pattern I that have a separate canalis nervus hyomandibularis distalis for the distal part of the nerve (e.g., Aldabrachelys gigantea, Gopherus polyphemus), or turtles with facial patterns IIA (e.g., Gopherus agassizii) or IIB (e.g., Pelomedusa subrufa) that have a hyomandibular sulcus irrespective of their canalis nervus hyomandibularis proximalis. 
The second source of variation not addressed by our ganglion position patterns comes from

1818

1819

1820

1821

1822

1823

1824

1825

1826

1827

1828

1829

1830

1831

1832

1833

1834

1835

1836

1837

1838

1839

the anteriormost (=distal) course of the vidian nerve, which passes through the canalis nervus

vidianus in the majority of turtles. The canalis nervus vidianus is present in all turtle clades but cheloniids and dermochelyids, in which the vidian nerve is inferred to extend through the canalis caroticus internus to the sulcus cavernosus, and then, in Dermochelys coriacea at least, to follow the path of the palatine artery (Nick, 1912). However, the path of the vidian nerve is highly variable within and between turtle clades, and several patterns can be identified, depending on whether the vidian nerve extends through the canalis caroticus internus, canalis caroticus lateralis, and sulcus cavernosus, or a combination of those. Those turtles classified in facial patterns IC and IIC have the vidian nerve that passes directly from the geniculate ganglion into the canalis nervus vidianus. In all other turtles, the vidian nerve extends through parts of the canal system that also houses the carotid arterial system. In podocnemidids (facial pattern IIA), the vidian nerve enters the cavum pterygoidei and likely extends close to the internal carotid artery before entering the canalis nervus vidianus. However, as the interaction between the vidian nerve and the internal carotid artery occurs within the cavum pterygoidei and is not documented in the bony skeleton, we cannot determine with certainty what the pattern for the vidian nerve is. In chelonioids, the vidian nerve is interpreted to follow the course of the palatine artery within the carotid arterial system, as no distinct canalis nervus vidianus can be observed. In turtles showing facial pattern IB, the vidian nerve passes directly into the palatine artery canal, from which it either directly enters the canalis nervus vidianus (the geoemydid Geoemyda spengleri), or first exits into the sulcus cavernosus before entering the canalis nervus vidianus (the emydid Glyptemys muhlenbergii). In turtles that exhibit facial patterns IA, IIA, or IIB, the vidian nerve always extends at least partially through the canalis caroticus internus. In these 
1840 turtles the vidian nerve extends from the canalis caroticus internus to the canalis nervus vidianus

1841 via one of five pathways (Fig. 20). In vidian pattern I, the vidian nerve enters a separate canalis

1842 nervus vidianus that directly branches off the internal carotid canal (Pelomedusa subrufa, chelids

1843 except for Chelus fimbriatus; Carettochelys insculpta, Platysternon megacephalum, all

1844 testudinids but those with pattern IC, the trionychid Cycloderma frenatum, the kinosternid

1845 Staurotypus salvinii, the chelydrid Chelydra serpentina, and the geoemydids Malayemys

1846 subtrijuga, Pangshura tecta, and Rhinoclemmys melanosterna). In vidian pattern II, the vidian

1847 nerve parallels the internal carotid artery up to its split, and then follows the palatine artery into

1848 its canal, from which a separate canalis nervus vidianus diverges (the emydids Graptemys

1849 geographica, Pseudemys floridana, Terrapene coahuila, Terrapene ornata). In vidian pattern III,

1850 the vidian nerve also parallels the palatine artery, but exits the respective canal through the

1851 foramen anterius canalis carotici lateralis into the sulcus cavernosus, from which a separate

1852 canalis nervus vidianus emerges (all trionychids but Cycloderma frenatum, all kinosternids but

1853 Staurotypus salvinii, Dermatemys mawii, the emydids Clemmys guttata, Emydoidea blandingii,

1854 and Emys orbicularis, and the geoemydids Batagur baska, Cuora amboinensis, Geoclemys

1855 hamiltonii, and Siebenrockiella crassicollis). In vidian patterns IV and V, the vidian nerve enters

1856 the canalis caroticus lateralis, but not via the arterial pathway, but instead via a 'shortcut' canal

1857 that connects the canalis caroticus internus and canalis caroticus lateralis. The vidian nerve then

1858 enters the canalis nervus vidianus that either branches directly off the canalis caroticus lateralis

1859 (vidian pattern IV; similar to vidian pattern II; the emydid Glyptemys insculpta), or emerges

1860 from the sulcus cavernosus after the vidian nerve has left the arterial canal via the foramen

1861 anterius canalis caroticus lateralis (vidian pattern V; similar to vidian pattern III; the geoemydids

1862 Mauremys leprosa and Morenia ocellata). The differences between these five vidian patterns are 
1863 relatively subtle and seem largely driven by the relative anteroposterior origin of the canalis

1864 nervus vidianus. This is possibly best exemplified by our examined specimen of the emydid

1865 Deirochelys reticularia (FMNH 98754), which exhibits an asymmetric pattern. On the left side

1866 of this specimen, the canalis nervus vidianus begins from the canalis caroticus internus (i.e.

1867 vidian pattern I), whereas on the right side, the canal splits from the canalis caroticus lateralis

1868 further anteriorly (i.e. vidian pattern II). Similarly, in a specimen of Cyclemys dentata (NHMUK

186997.11 .22 .3 ), the exit of the canalis nervus vidianus is asymmetric, as it extends into the sulcus

1870 cavernosus on the left side, but connects to the canalis caroticus lateralis on the right side. Our

1871 patterns of the relative position of the canalis nervus vidianus are not easily matched with the

1872 phylogenetic relationships of turtles, so that the observed variation between taxa does not seem

1873 to be systematic.

1874

1875 Inferring canal homology from osteological material

1876 Our study broadly confirms that the canals that penetrate the basicranium of turtles can be

1877 identified correctly based on osteological material alone. The greatest source of past error

1878 pertains to the correct identity of the lateral canal versus the vidian canal. As outlined throughout

1879 this contribution, these two canals can be distinguished clearly by a series of topological criteria.

1880 The lateral carotid canal typically splits from the internal carotid near the basisphenoid-pterygoid

1881 suture, extends parallel to the basisphenoid-pterygoid suture and sulcus cavernosus, with which it

1882 merges. The vidian canal, by contrast, originates from the facial nerve canal at the likely position

1883 of the geniculate ganglion, punctures the pterygoid and/or palatine, crosses the path of the sulcus

1884 cavernosus, and is directed towards the foramen palatinum posterius. These criteria are sufficient 
1885 to correctly identify the single canal in all extant turtles, with two notable exceptions:

1886 podocnemidis and chelids.

1887 The dissection studies of Albrecht $(1967,1976)$ highlight that the fenestra located between the

1888 cavum pterygoidei and the sulcus cavernosus of podocnemidids does not hold the palatine artery,

1889 as this blood vessel is absent. This situation is troubling, as a passage for the palatine artery is

1890 available at the right location, but not used. However, the morphology of this potential passage is

1891 very unlike for a regular blood or nerve canal, but rather approximates many other poorly

1892 defined opening, such as the foramen orbito-nasale, hiatus acusticus, or the fenestra caroticus by

1893 lacking clear evidence for the former passage of a structure. Nevertheless, we suggest scoring the

1894 lateral canal (or any canal) for fossil podocnemidis as unknown wherever a passage is apparent

1895 that lacks the characteristics of a true blood foramen.

1896 In some chelids, a canal exists that connects the internal carotid canal with the sulcus

1897 cavernosus, but the dissection studies of Albrecht $(1967,1976)$ suggest that this is the vidian

1898 canal, not the lateral canal. Although this canal often connects with the geniculate ganglion and

1899 has the same diameter as a more anterior canal with unambiguous vidian affiliations (see Fig. 3

1900 for Chelus fimbriatus), its passage close to the basisphenoid-pterygoid suture is more

1901 characteristic of a lateral canal. As for fossil podocnemidids, we recommend scoring fossil

1902 chelids as unknown for the presence of a lateral canal. For the unlikely event that a similar

1903 arrangement is found for a fossil group of turtles unrelated to chelids, we suggest scoring the

1904 lateral and vidian canals as unknown, whenever true evidence for a vidian canal only is lacking

1905 (e.g., a path deep within the pterygoid or continuous connection of the geniculate ganglion with

1906 the foraman palatinum posterius).

1907 


\section{Canal sizes}

1909 The carotid arterial system is the sole source of blood to the cranium and its subordinate

1910

1911

1912

1913

1914

1915

1916

1917

1918

1919

1920

1921

1922

1923

1924

1925

1926

1927

1928

1929

1930

arteries supply all major cranial tissues. The majority of cranial tissues are supplied by branches of the internal carotid artery, but some parts are also supplied by the branches of the external carotid artery (e.g., Kardong, 1998). Generally, in reptiles, the anterior head region, including the orbit, is supplied by two branches of the common carotid artery: the stapedial artery and at least one major subordinate branch of the medially directed carotid branch that otherwise supplies the brain via the cerebral artery. The subordinate, facially-directed branch of the brain-supplying internal carotid branch is either the orbital artery, which branches off the cerebral artery from within the sella turcica, or the sphenopalatine artery, which branches off the cerebral/internal carotid before the latter enters the basisphenoid (e.g., Albrecht, 1967; Porter \& Witmer, 2015; Porter, Sedlmayr \& Witmer, 2016). The orbital and sphenopalatine arteries can co-exist (e.g., crocodiles; Sedlmayr, 2002; Porter \& Witmer, 2015; most turtles: Albrecht, 1967), but one of these arteries usually dominates in terms of arterial size, and thus blood volume transmitted, so that only one 'medial' artery is of volumetric importance for the blood supply of the anterior head region. The sphenopalatine artery of turtles is generally called the palatine artery, when present (e.g., Albrecht, 1967; Gaffney, 1979). The orbital artery is usually also present in turtles, albeit as a small artery (Albrecht, 1967). One exception are trionychids, in which the orbital artery (i.e., a branch of the cerebral artery that originates in the sella turcica) is exceptionally large and bifurcates anteriorly within the orbital cavity to supply the orbit (Albrecht, 1967). Albrecht (1967) used the neologism 'pseudopalatine artery' for the orbital artery of trionychids, although the origination within the sella turcica and from the cerebral artery, as well as its direction toward the orbit are consistent with its identification as the orbital artery, an artery that 
1931 Albrecht (1967) had also identified in other turtle groups. Thus, the major blood supply for the 1932 anterior head region, including the orbit, is achieved by three different arteries in turtles: either 1933 by both the palatine artery and the stapedial artery (when both arteries are well developed, e.g., 1934 cheloniids: Albrecht, 1976), or predominantly by the stapedial artery (when the palatine artery is 1935 absent or small, e.g., Chrysemys: Albrecht, 1967), or predominantly by the palatine artery (when the stapedial artery is reduced or absent, e.g., Sternotherus: Albrecht, 1967), or predominantly by 1937 the orbital artery (when the palatine artery is absent and stapedial artery is reduced in size, e.g., 1938 trionychids: Albrecht, 1967). Although the origin of the arteries suppling the anterior skull 1939 region thus varies across turtles, the terminal patterning of these arteries, such as the development of supra- and infraorbital arteries, are largely consistent (see Albrecht, 1967). Variation as to which artery supplies specific organs is also documented for the mandible in turtles: whereas in non-turtle reptiles and many turtles, the mandibular artery originates as a branch of the stapedial artery (e.g., Albrecht, 1967, 1976; Gaffney, 1979; Porter \& Witmer, 2015), the mandibular artery in some turtles may also branch from the palatine artery when the stapedial artery is reduced (e.g., Sternotherus odoratus: Albrecht, 1967) or the internal carotid artery when the stapedial and palatine arteries are reduced (e.g., trionychids: Albrecht, 1967). different clades (e.g., Albrecht, 1967, 1976; Gaffney, 1979; this study). As the above examples show, these arterial reductions affect the patterns of blood supply, as the arteries that supply the anterior head or mandible regions are always present but have different branching points along more proximal parts of the carotid arterial system, depending on which arteries are reduced and which are not. Based on his dissections of a few representatives of most turtle clades, Albrecht 1953 (1976) hypothesized that reductions in the size of the stapedial artery are counterbalanced by 
1954 increases in size of the palatine or orbital arteries to ensure the arterial blood supply for the 1955 anterior region of the head. Although relatively many studies related to the cranial arteries or 1956 canals are available, and although qualitative observations for various turtle clades seem to 1957 provide support for Albrecht's (1976) hypothesis (e.g., McDowell, 1961), only few publications 1958 explicitly compare or quantify canal sizes for a large number of species (e.g., Jamniczky \& Russell, 2007). Here, we digitally measured the cross-sectional area of the canalis caroticus internus, the canalis caroticus lateralis, the canalis caroticus basisphenoidalis, and the canalis stapedio-temporalis in our CT-scans (see Methods for further details). These measurements, summarized in Table 2, provide approximate estimates of blood flow, as arterial canal diameter is proportional to arterial size in turtles (Albrecht, 1976; Jamniczky \& Russell, 2004).

Our pGLS analysis finds strong evidence of a correlation between internal carotid artery size and stapedial artery size (Table 5). Variation in cross-sectional area of the stapedial artery canal explains a large portion of variance in the cross-sectional area of the internal carotid artery canal $\left(\mathrm{R}^{2}=0.73\right)$. The regression line has an intercept of 1.097 and a slope of 0.776 (Fig. 21A; Table 5), indicating a moderately negative allometric scaling relationship between medial and lateral arterial sizes. The relationship between cross-sectional areas of stapedial and internal carotid arteries underwent evolutionary change on the tree, as the phylogenetic signal of this relationship as estimated during the fitting of the regression model is high (lambda $=0.96$ ). trionychians, kinosternoids, and chelonioids (Fig. 21B-21C). These clades have larger internal carotid cross-sectional areas than expected by the regression, although this effect is much smaller in chelonioids than in trionychids and kinosternoids, which are visually separated from the remaining data (Fig. 21A), but seem to follow a trend parallel to the remaining data. To test if 
1977 our data can be better explained by a model with different intercepts or slopes or both for the

1978 visually separated groups, we performed generalized least-squares phylogenetic analysis of

1979 covariance (pANCOVA) using the gls.ancova function of the package evomap (Smaers \& Rohlf, 1980 2016; Smaers \& Mongle, 2018). We defined a group with consistently positive residuals (i.e.

1981 chelonioids, kinosternoids, trionychians) and another with the remaining taxa for which models

1982 could be compared. Results of the pANCOVA show that a model with varying intercepts is 1983 significantly supported over a single intercept-single slope model $(\mathrm{F}$ value $=14.53, \mathrm{p}<0.0003)$, 1984 and that this model is favored over a model with varying slopes and intercept $(\mathrm{F}=3.53, \mathrm{p}=$ 1985 0.06). These results demonstrate that kinosternoids, chelonioids, and trionychians follow a 1986 regression line with an elevated intercept in comparison to other turtles (Fig. 21B; Table 5). 1987 These taxa have larger internal carotid cross-sectional areas than other turtles (as the intercept is 1988 elevated), but show the same proportional size increase (allometric scaling relationship) with 1989 increasing stapedial artery size than other turtles (as regression slopes are near identical). These 1990 observations quantitatively confirm previous hypotheses, as particularly trionychids and 1991 kinosternoids are known for their small stapedial artery canals. However, and somewhat 1992 unexpectedly, the same is also true for some taxa with relatively large stapedial arteries, such as carettochelyids or chelonioids, indicating that these taxa have a greater 'medial' blood flow than expected based on their stapedial artery sizes, which are relatively large to begin with. Much of the variation we observe can be attributed to the placement of the mandibular artery, which draws blood from the carotid system, but does not supply the anterior head region. In trionychids and kinosternoids, the mandibular artery is supplied by the lateral branch of the internal carotid artery (Albrecht, 1967, 1976). It is therefore not surprising, that the diameter of the internal carotid artery is larger than expected. The high residuals found in Carettochelys insculpta, 
2000 however, cannot be explained by this model, as our observations indicate that the mandible of

2001 this taxon is supplied by the stapedial artery prior to its passage through the stapedial canal. In

2002 chelonioids, the mandible is partially supplied by the stapedial artery, but prior to its passing

2003 through the stapedial canal, and by the lateral branch of the internal carotid artery (Albrecht,

2004 1976). The internal carotid artery is therefore enlarged again, but not to the extent as seen in

2005 trionychids and kinosternids. The opposite situation is seen in chelids, where the mandible is

2006 completely supplied by the stapedial artery after its passage through the stapedial canal

2007 (Albrecht, 1976). The stapedial canal is therefore disproportionaly enlarged. A similar

2008 enlargement, however, is not apparent for the remaining pleurodires, even though mandibles are

2009 fully (Pelomedusidae) or partially (Podocnemididae) supplied by the stapedial artery following

2010 its passage through the stapedial canal (Albrecht, 1976). In all remaining turtles, the mandible is

2011 supplied by the stapedial artery, but prior to its passage through the stapedial canal (Albrecht,

20121967 1976). In summary, our canal size data quantitatively confirm that variation in the stapedial

2013 artery size inversely correlates with the internal carotid artery size and that departure from the

2014 mean can partially be explained by the configuration of the mandibular artery.

2015

2016 Evolution of the internal carotid arterial system of turtles

2017 Plesiomorphically, the split of the internal carotid artery into its cerebral and palatal branches

2018 occurs extracranially (i.e. not embedded in bone), with the palatine branch extending anteriorly

2019 through the interpterygoid vacuity, and the cerebral artery extending through the basisphenoid.

2020 This condition is known in basal taxa such as Proganochelys quenstedtii and Kayentachelys

2021 aprix (Gaffney, 1990; Sterli \& Joyce, 2007; Gaffney \& Jenkins, 2010). The plesiomorphic

2022 presence of the palatine artery, which leaves no direct osteological correlate in these turtles, is 
2023 inferred based on outgroup comparisons (Müller, Sterli \& Anquetin, 2011), and the presence of

2024 palatine artery canals in slightly derived stem turtles with closed interpterygoid openings.

2025 Examples of those are Kallokibotion bajazidi (Gaffney \& Meylan, 1992) and Mongolochelys

2026 efremovi (Sterli et al., 2010), in which the split of the internal carotid artery into its cerebral and

2027 palatal branches still occurs extracranially, but with the closure of the interpterygoid vacuity, a

2028 distinct canalis caroticus lateralis is present. As all extant turtles have the internal carotid artery

2029 embedded in bone posterior to its split, this condition could be symplesiomorphic for crown-

2030 group turtles and may possibly have evolved somewhere on the stem-lineage of turtles.

2031 Examination of fossil representatives of the stem-lineages for the major turtle subclades will

2032 provide important clues as to whether the complete embedding of the carotid system in bone is

2033 indeed ancestral for the crown-group. The evolutionary reconstruction of the carotid embedding

2034 is also hindered by varying phylogenetic hypotheses regarding the position of several turtle

2035 clades as stem or crown-group turtles, including paracryptodires, thalassochelydians,

2036 xinjiangchelyids, and sinemydids/macrobaenids (e.g., Joyce, 2007; Sterli, 2010; Anquetin, 2012;

2037 Zhou \& Rabi, 2015; Cadena \& Parham, 2015; Joyce et al., 2016; Evers \& Benson, 2019). Partial

2038 embedding of the internal carotid artery is observed in macrobaenids/sinemydids such as

2039 Dracochelys bicuspis (Gaffney \& Ye, 1992), xinjiangchelyids like Xinjiangchelys wusu (Rabi et

2040 al., 2013), or thalassochelydians like Plesiochelys etalloni (NMS 40870). These turtles all share

2041 that the posterior part of the internal carotid is embedded in bone, but the anterior part around the

2042 area of the split into palatine and cerebral arteries is exposed in a fenestra caroticus (Rabi et al.,

2043 2013). If these turtles are evolutionary intermediates between the earliest known turtles and

2044 crown-group turtles with completely embedded carotids, as indicated by some phylogenetic

2045 analyses (e.g., Evers \& Benson, 2018), their morphology indicates that the encasing of the 
2046 carotid artery followed a 'posterior-section-first'-pattern. The converse pattern is observed 2047 among some paracryptodires: In the baenid Eubaena cephalica, a short anterior section of the 2048 internal carotid artery prior to its entry into the basisphenoid (upon which it becomes the cerebral 2049 artery), is embedded in bone (Rollot, Lyson \& Joyce, 2018), whereas the palatine artery is 2050 absent. This could provide evidence for an 'anterior-section-first' embedding of the internal 2051 carotid artery. However, it is questionable if the baenid condition is truly informative about the 2052 evolution of crown-group turtles. Baenids are most frequently inferred to be deeply nested within 2053 Paracryptodira (e.g., Lyson \& Joyce, 2011), but pleurosternids and some of the earliest 2054 paracryptodires, such as Uluops uluops, have carotid patterns quite distinct from those of baenids 2055 (e.g., Anquetin \& André, 2020; Evers, Rollot \& Joyce, in review). In Uluops uluops, the carotid 2056 split seems to be exposed (pers. obs. UCM 53971), indicating that no foramen posterius canalis 2057 carotici interni is present. The same condition has been reported for Dorsetochelys typocardium 2058 (Anquetin \& André, 2020). In pleurosternids, the far-anteriorly positioned foramen posterius 2059 canalis carotici interni has likely been misidentified, and the visible foramen in the suture of the

2060

2061

2062

2063

2064

2065

2066

2067 2068 basisphenoid and pterygoid is for the cerebral artery instead, whereas the palatine artery is likely lost (pers. obs. UMZC T1041: Pleurosternon bullockii; Evers, Rollot \& Joyce, in review). Thus, Uluops and pleurosternids possibly have entirely uncovered internal carotid arteries and therefore the plesiomorphic condition of Testudinata. Depending on paracryptodiran in-group relationships, the loss of the palatine branch of the internal carotid artery has occurred independently at least one time within paracryptodires (Rollot, Lyson \& Joyce, 2018), and several times within both extant lineages.

The use of CT data will probably yield new results about the cranial morphology and circulation system of turtles. Particularly important will be the inclusion of fossil taxa belonging 
2069 to the stem of lineages of the primary clades within Testudines (i.e. former families or

2070 superfamilies such as Trionychia, Geoemydidae, Chelonioidea, etc.), as those will provide new

2071 insights into evolutionary changes during the early diversification of turtles.

2072

2073 CONCLUSIONS

2074 We here describe the carotid circulation and facial nerve systems of all major extant clades of 2075 turtles based on micro-CT scans of 69 specimens representing 65 species. Our main results 2076 include reinterpretations of the carotid arterial canal system and the facial nerve system and show 2077 that some canals pertaining to the facial nerve system have been misinterpreted in the past as 2078 carotid canals. Our observations warrant nomenclatural updates to previous canal definitions, 2079 which facilitate the precise description of the observed disparity. We demonstrate that the 2080 complete loss of the palatine artery and the respective canalis caroticus lateralis is more 2081 widespread among turtles than previously recognized, and happened independently in 2082 pleurodires, carettochelyids, platysternids, testudinids, and some geoemydids. Quantitative canal 2083 size data shows that variation of stapedial vs. non-stapedial carotid canal size can largely be 2084 attributed to differences in mandible artery course. We review these differences across turtle 2085 groups and add novel observation regarding osteological correlates for the mandibular artery, 2086 which help to distinguish mandibular blood supply patterns in cryptodires even in the absence of 2087 direct arterial observation. Our data furthermore shows unexpected variation with regard to the 2088 canal system for the facial nerve, particularly with respect to the position of the geniculate 2089 ganglion, as well as the course of the subordinate facial nerve branches, the vidian and 2090 hyomandibular nerves through their specific canals. These data show that previously 2091 hypothesized distinctions in the facial nerve system of pleurodires and cryptodires cannot be 
2092 upheld. The carotid artery and facial nerve systems appear to vary independently from each

2093 other, although the facial nerve and carotid systems in part share the same canals. The carotid

2094 circulation and facial nerve systems have been used as a source of phylogenetic characters in

2095 many studies, but our observations provide the basis for the revision of previously phrased

2096 characters and the conception of new phylogenetic characters. Important future tasks include the

2097 integration of fossil data both from stem-lineages of extant groups to facilitate understanding of

2098 the evolution of modern diversity regarding these systems, as well from stem-turtles to

2099 understand the evolution of the derived arterial embedding found in turtles.

2100

2101

\section{ACKNOWLEDGEMENTS}

2102 We would like to acknowledge the curators and staff of museums that provided us access to 2103 specimens used in this study, particularly Loïc Costeur (NMB), Alan Resetar (FMNH), Patrick

2104 Campbell (NHMUK), Linda Mogk (SMF), Alexander Kupfer (SMNS), and Jason Head

2105 (UMZC). We thank research and technical staff and PIs at the CT scanning facilities we used for

2106 this research, namely Tom Davies and Ben Moon (University of Bristol), Farah Ahmed

2107 (NHMUK), Zhe-Xi Luo and April Isch Neander (University of Chicago), and Ingmar Werneburg

2108 (University of Tübingen). We also extend our gratitude to the contributors and curators of

2109 MorphoSource. We finally thank Guilherme Hermanson (University of São Paulo) for making a

2110 specimen of Podocnemis expansa available for additional examination. Gabriel Ferreira, Heather

2111 Jamniczky, Olivier Rieppel, and an anonymous reviewer are thanked for numerous thoughtful

2112 comments that greatly helped improve the quality of this manuscript.

\section{REFERENCES}


2115 Albrecht PW. 1967. The cranial arteries and cranial arterial foramina of the turtle genera

2116 Chrysemys, Sternotherus and Trionyx: a comparative study of analysis with possible

2117 evolutionary implications. Tulane Studies in Zoology 14:81-99.

2118 Albrecht PW. 1976. The cranial arteries of turtles and their evolutionary significance. Journal of

2119 Morphology 149:159-182.

2120 Anquetin J, André C. 2020. The last surviving Thalassochelydia - a new turtle cranium from

2121 the Early Cretaceous of the Purbeck Group (Dorset, UK). PaleorXiv.

2122 doi:10.31233/osf.io/7pa5c.

2123 Anquetin J. 2012. Reassessment of the phylogenetic interrelationships of basal turtles

2124 (Testudinata). Journal of Systematic Palaeontology 10:3-45.

2125 Bardet N, Jalil N-E, Lapparent de Broin F de, Germain D, Lambert O, Amaghzaz M. 2013.

2126 A giant chelonioid turtle from the Late Cretaceous of Morocco with a suction feeding

2127 apparatus unique among tetrapods. PLOS ONE 8:1-10.

2128 Bender O. 1906. Die Schleimhautnerven des Facialis, Glassopharyngeus und Vagus. Jenaische

2129 Denkschriften 7:343-454.

2130 Benoit J, Manger PR, Rubidge BS. 2016. Palaeoneurological clues to the evolution of defining

2131 mammalian soft tissue traits. Scientific Reports 6:25604.

2132 Benoit J, Angielczky KD, Miyamae JA, Manger P, Fernandez V, Rubridge B. 2018.

2133 Evolution of facial innervation in anomodont therapsids (Synapsida): Insights from X-ray

2134 computerized microtomography. Journal of Morphology 279:673-701.

2135 Bojanus LH. 1819-1821. Anatome testudinis europaeae. Vilna: Joseph Sawadzki.

2136 Brinkman DB, Peng JH. 1993. New material of Sinemys (Testudines, Sinemydidae) from the 2137 early cretaceous of China. Canadian Journal of Earth Sciences 30:2139-2152. 
2138 Brinkman DB, Peng JH. 1996. A new species of Zangerlia (Testudines: Nanhsiungchelyidae)

2139 from the Upper Cretaceous redbeds and Bayan Mandahu, Inner Mongolia, and the

2140 relationships of the genus. Canadian Journal of Earth Sciences 33:526-540.

2141 Cadena E, Parham JF. 2015. Oldest known marine turtle? A new protostegid from the Lower 2142 Cretaceous of Colombia. PaleoBios 32:1-421.

2143 Crawford NG, Parham JF, Sellas AB, Faircloth BC, Glenn TC, Papenfuss TJ, Henderson

2144 JB, Hansen MH, Simison WB. 2015. A phylogenomic analysis of turtles. Molecular

$2145 \quad$ Phylogenetics and Evolution 83:250-257.

2146 Crumly CR. 1982. A cladistic analysis of Geochelone using cranial osteology. Journal of

2147 Herpetology 16:215-234.

2148 Crumly CR. 1994. Phylogenetic systematics of North American tortoises (Genus Gopherus):

2149 Evidence for their classification. Fish and Wildlife Research 13:7-32.

2150 Dehnhardt G, Mauck B. 2008. Mechanoreception in secondarily aquatic vertebrates. In:

2151 Thewissen JGM, Nummela S, eds. Sensory evolution on the threshold: adaptations in

2152 secondarily aquatic vertebrates. Berkeley: University of California Press, 295-314.

2153 Evans SE. 1987. The braincase of Youngina capensis (Reptilia: Diapsida; Permian). Neues

2154 Jahrbuch für Geologie und Paläontologie-Monatshefte 4:193-203.

2155 Evers SW. 2019. CT scans of extant turtle skulls. MorphoSource. Available at

2156 https://www.morphosource.org/Detail/ProjectDetail/Show/project_id/769.

2157 Evers SW, Barrett PM, Benson RBJ. 2019. Anatomy of Rhinochelys pulchriceps

2158 (Protostegidae) and marine adaptation during the early evolution of chelonioids. PeerJ

$2159 \quad 7: \mathrm{e} 6811$ 
2160 Evers SW, Benson RBJ. 2018. Project: Evers \& Benson 2018, Turtle CT data and 3D models.

$2161 \quad$ MorphoSource. Available at

2162 http://www.morphosource.org/Detail/ProjectDetail/Show/project_id/462.

2163 Evers SW, Benson RBJ. 2019. A new phylogenetic hypothesis of turtles with implications for

2164 the number of evolutionary transitions to marine lifestyles supports an Early Cretaceous

2165 origin and rapid diversification of Chelonioidea. Palaeontology 62:93-134.

2166 Evers S, Joyce WG. 2020. A re-descripton of Sandownia harrisi (Testudinata: Sandownidae)

2167 from the Aptian of the Isle of Wight based on computed tomography scans. Royal Society

$2168 \quad$ Open Science 7:191936.

2169 Evers S, Rollot Y, Joyce WG. 2020. Cranial osteology of the Early Cretaceous turtle

$2170 \quad$ Pleurosternon bullockii (Paracryptodira: Pleurosternidae).

2171 Felsenstein J. 1985. Phylogenies and the Comparative Method. American Naturalist 125:1-15.

2172 Fuchs H. 1931. Ueber den Unterkiefer und die Unterkiefernerven (Ramus tertius nervi trigemini

2173 et Chorda tympani) der Arrauschildkröte (Podocnemis expansa), nebst Bemerkungen zur

2174 Kiefergelenksfrage. Zeitschrift für Anatomie und Entwicklungsgeschichte 94:206-274.

2175 Gaffney ES. 1972. An illustrated glossary of turtle skull nomenclature. American Museum

$2176 \quad$ Novitates 2486:1-33.

2177 Gaffney ES. 1975. A phylogeny and classification of the higher categories of turtles. Bulletin of 2178 the American Museum of Natural History 155:387-436.

2179 Gaffney ES. 1976. Cranial morphology of the European Jurassic turtles Portlandemys and 2180 Plesiochelys. Bulletin of the American Museum of Natural History 157:487-544.

2181 Gaffney ES. 1979. Comparative cranial morphology of recent and fossil turtles. Bulletin of the 2182 American Museum of Natural History 164:69-376. 
2183 Gaffney ES. 1983. The cranial morphology of the extinct horned turtle Meiolania platyceps,

2184 from the Pleistocene of Lord Howe Island, Australia. Bulletin of the American Museum of

$2185 \quad$ Natural History 175:361-480.

2186 Gaffney ES. 1990. The comparative osteology of the Triassic turtle Proganochelys. Bulletin of 2187 the American Museum of Natural History 194:1-176.

2188 Gaffney ES. 1996. The postcranial morphology of Meiolania platyceps and a review of the 2189 Meiolaniidae. Bulletin of the American Museum of Natural History 229:1-166.

2190 Gaffney ES, Jenkins FA. 2010. The cranial morphology of Kayentachelys, an Early Jurassic 2191 cryptodire, and the early history of turtles. Acta Zool-Stockholm 91:335-368.

2192 Gaffney ES, Meylan PA. 1988. A phylogeny of turtles. In: Benton MJ, ed. The phylogeny and 2193 classification of the tetrapods, volume 1: amphibians, reptiles, birds. Oxford: Clarendon 2194 Press, 157-219.

2195 Gaffney ES, Meylan PA. 1992. The Transylvanian turtle Kallokibotion, a primitive cryptodire 2196 of cretaceous age. American Museum Novitates 3040:1-37.

2197 Gaffney ES, Meylan PA \& Wyss AR. 1991. A computer assisted analysis of the relationships 2198 of the higher categories of turtles. Cladistics 7:313-335.

Gaffney ES, Rich TH, Vickers-Rich P, Constantine A, Vacca R, Kool L. 2007. Chubutemys, a new eucryptodiran turtle from the early cretaceous of Argentina, and the relationships of Meiolaniidae. American Museum Novitates 3599:1-35.

Gaffney ES, Tong H, Meylan PA. 2006. Evolution of the side-necked turtles: the families Bothremydidae, Euraxemydidae, and Araripemydidae. Bulletin of the American Museum of Natural History 300:1-698. 
2205 Gaffney ES, Ye X. 1992. Dracochelys, a new cryptodiran turtle from the early cretaceous of

2206 China. American Museum Novitates 3048:1-13.

2207 Garbin RC, Ascarrunz E, Joyce WG. 2018. Polymorphic characters in the reconstruction of 2208 the phylogeny of geoemydid turtles. Zoological Journal of the Linnean Society 184:896-918.

2209 Gaupp E. 1888. Anatomische Untersuchungen über die Nervenversorgung der Mund- und 2210 Nasenhöhlendrüsen der Wirbeltiere. Morphologisches Jahrbuch 14:436-489.

2211 George ID, Holliday CM. 2013. Trigeminal nerve morphology in Alligator mississippiensis and 2212 its significance for crocodyliform facial sensation and evolution. Anatomical Record $2213 \quad 296: 670-680$.

2214

2215

2216

Grafen A. 1989. The phylogenetic regression. Philosophical Transactions of the Royal Sociey of London B: Biological Sciences 326:119-157.

Hanson FB. 1919. The anterior cranial nerves of Chelydra serpentina. Washington University Studies, Science Series 7:13-41.

Haughton SH. 1929. Notes on some pareiasaurian brain-cases. Annals of the South African Museum 28:88-96.

Hermanson G, Iori FV, Evers SW, Langer MC, Ferreira GS. 2020. A small podocnemidoid (Pleurodira, Pelomedusoides) from the Late Cretaceous of Brazil, and the innervation and carotid circulation of side-necked turtles. Papers in Paleontology 6:329-347

Hirayama R. 1998. Oldest known sea turtle. Nature 392:705-708.

Hoffmann CK. 1890. Reptilien. I. Schildkröten. In: Bronn HG, ed. Klassen und Ordnungen des Thier-Reichs, Vol. 6. Leipzig: C. F. Winter'sche Verlagshandlung, 1-442.

Hooks GE. 1998. Systematic revision of the Protostegidae, with a redescription of Calcarichelys gemma Zangerl, 1953. Journal of Vertebrate Paleontology 18:85-98 
2228 Ives AR. 2018. R2s for correlated data: phylogenetic models, LMMs, and GLMMs. Systematic 2229 Biology 68:234-251.

2230

2231

2232

2233

2234

2235

2236

2237

2238

2239

2240

2241

2242

2243

2244

2245

2246

2247

2248

2249

HA Jamniczky, AP Russell. 2004. Cranial arterial foramen diameter in turtles: a quantitative assessment of size-independent phylogenetic signal. Animal Biology 54:417-436.

HA Jamniczky, AP Russell. 2007. Reappraisal of patterns of nonmarine cryptodiran turtle carotid circulation: evidence from osteological correlates and soft tissues. Journal of Morphology 268:571-587.

Jamniczky HA. 2008. Turtle carotid circulation: a character analysis case study. Biological Journal of the Linnean Society 93:239-256.

Joyce WG. 2007. Phylogenetic relationships of Mesozoic turtles. Bulletin of the Peabody Museum of Natural History 48:3-102.

Joyce WG, Rabi M, Clark JM, Xu X. 2016. A toothed turtle from the Late Jurassic of China and the global biogeographic history of turtles. BMC Evolutionary Biology 16:236.

Joyce WG, Volpato VS, Rollot Y. 2018. The skull of the carettochelyid turtle Anosteira pulchra from the Eocene (Uintan) of Wyoming and the carotid canal system of carettochelyid turtles. Fossil Record 21:301-310.

Kenneth V. 1998. Vertebrates: Comparative Anatomy, Function, Evolution. 2nd Edition.

$$
\text { Boston, Massachusetts, McGraw-Hill. }
$$

Kear BP, Lee MSY. 2006. A primitive protostegid from Australia and early sea turtle evolution.

$$
\text { Biology Letters 2:116-119. }
$$

Kesteven HL. 1910. The anatomy of the head of the green turtle Chelone midas, Latr. Part I. The skull. Journal and Proceedings of the Royal Society of New South Wales 44:368-400. 
2250 Lapparent de Broin F de, Werner C. 1998. New late Cretaceous turtles from the Western

2251 Desert, Egypt. Annales de Paléontologie 84:131-214.

2252 Lapparent de Broin F de, de la Fuente MS, Fernandez MS. 2007. Notoemys laticentralis

2253 (Chelonii, Pleurodira), Late Jurassic of Argentina: new examination of the anatomical

2254 structures and comparisons. Revue de Paléobiologie 26:99-136.

2255 Lautenschlager S, Ferreira GS, Werneburg I. 2018. Sensory evolution and ecology of early

2256 turtles revealed by digital endocranial reconstructions. Frontiers in Ecology and Evolution

$2257 \quad 6: 7$.

2258 Lawson R. 1970. The caecilian cardio-vascular system and intra-amphibian relationships.

2259 Journal of Zoology 160:199-229.

2260 Lipka TR, Therrien F, Weishampel DB, Jamniczky HA, Joyce WG, Colbert MW,

2261 Brinkman DB. 2006. A new turtle from the Arundel Clays (Potomac Formation, Early

2262 Cretaceous) of Maryland, U.S.A. Journal of Vertebrate Paleontology 26:300-307.

2263 Lyson TR, Joyce WG. 2011. Cranial anatomy and phylogenetic placement of the enigmatic

2264 turtle Compsemys victa. Journal of Paleontology 85:789-801.

2265 McDowell SB. 1961. On the major arterial canals in the ear region of testudinoid turtles and the

2266 classification of the Testudinoidea. Bulletin of the Museum of Comparative Zoology 125:23-

$2267 \quad 39$.

2268 Meylan PA. 1987. The phylogenetic relationships of soft-shelled turtles (family Trionychidae).

2269 Bulletin of the American Museum of Natural History 186:1-110.

2270

Meylan PA, Gaffney ES. 1989. The skeletal morphology of the Cretaceous cryptodiran turtle,

2271 Adocus, and the relationships of the Trionychoidea. American Museum Novitates 2941:1-60. 
2272 Miyashita T. 2012. Geometric and developmental perspectives on the evolution of the skull and

2273 internal carotid circulation in turtles. In: Brinkman DB, Holroyd PA, Gardner JD, eds.

2274 Morphology and Evolution of Turtles, Vertebrate Paleobiology and Paleoanthropology.

2275 Dordrecht: Springer Science+Business Media, 71-101.

2276

2277

Motani R, Schmitz L. 2011. Phylogenetic functional signals in the evolution of form-function

2278 relationships in terrestrial vision. Evolution 65:2245-2257.

2279

Muchlinski MN. 2008. The relationship between the infraorbital foramen, infraorbital nerve, and maxillary mechanoreception: implications for interpreting the paleoecology of fossil mammals based on infraorbital foramen size. Anatomical Record 291:1221-1226.

2281

2282

2283

Müller J, Sterli J, Anquetin J. 2011. Carotid circulation in amniotes and its implications for turtle relationships. Neues Jahrbuch für Geologie und Paläontologie, Abhandlungen 261:289-297.

2284

2285

Nagelkerke NJD. 1991. A note on a general definition of the coefficient of determination. Biometrika 78:691-692.

Nick L. 1912. Das Kopfskelet von Dermochelys coriacea L. Zoologische Jahrbücher, Abteilung für Anatomie und Ontogenie der Tiere 33:431-552.

2288

2289

2290

Noack T. 1906. Über die Entwicklung des Mittelohres von Emys europaea nebst Bemerkungen zur Neurologie dieser Schildkröte. Archiv für Mikroskopische Anatomie 69:457-490.

O'Keefe R. 2001. A cladistic analysis and taxonomic revision of the Plesiosauria (Reptilia: Sauropterygia). Acta Zoologica Fennica 213:1-63.

Oelrich TM. 1956. The Anatomy of the Head of Ctenosaura pectinata (Iguanidae). Miscellaneous Publications Museum of Zoology, University of Michigan 94:1-122. 
2294 Ogushi K. 1911. Anatomische Studien an der japanischen dreikralligen Lippenschildkröte

2295

2296

2297

2298

2299

2300

2301

2302

2303

2304

2305

2306

2307

2308

2309

2310

2311

2312

2313

2314

2315

(Trionyx japanicus). I. Mitteilung. Morphologisches Jahrbuch 43:1-106

Ogushi K. 1913a. Anatomische Studien an der japanischen dreikralligen Lippenschildkröte

(Trionyx japanicus). II. Mitteilung: Muskel- und peripheres Nervensystem. Morphologisches Jahrbuch 46:299-562

Ogushi K. 1913b. Zur Anatomie der Hirnnerven und des Kopfsympathicus von Trionyx japonicus nebst einigen kritischen Bemerkungen. Morphologisches Jahrbuch 45:441-480

Pagel MD. 1997. Inferring evolutionary processes from phylogenies. Zoologica Scripta 26:331348.

Pagel MD. 1999. Inferring the historical patterns of biological evolution. Nature 401:877-884.

Paradis E, Schliep K. 2018. ape 5.0: an environment for modern phylogenetics and evolutionary analyses in R. Bioinformatics 35:526-528.

Pardo JD, Anderson JS. 2016. Cranial morphology of the Carboniferous-Permian tetrapod Brachydectes newberryi (Lepospondyli, Lysorophia): new data from $\mu \mathrm{CT}$. PLoS ONE 11:e0161823.

Paulina-Carabajal A, Sterli J, Georgi J, Poropat SF, Kear BP. 2017. Comparative neuroanatomy of extinct horned turtles (Meiolaniidae) and extant terrestrial turtles (Testudinidae), with comments on the palaeobiological implications of selected endocranial features. Zoological Journal of the Linnean Society 180:930-950.

Pereira AG, Sterli J, Moreira FRR, Schrago CG. 2017. Multilocus phylogeny and statistical biogeography clarify the evolutionary history of major lineages of turtles. Molecular Phylogenetics and Evolution 113:59-66 
2316 Porter WR, Witmer LM. 2015. Vascular patterns in iguanas and other squamates: blood

2317 vessels and sites of thermal exchange. PLoS ONE 10:e0139215.

2318 Porter WR, Sedlmayr JC, Witmer LM. 2016. Vascular patterns in the heads of crocodilians:

2319 blood vessels and sites of thermal exchange. Journal of Anatomy 229:800-824.

2320 Rabi M, Zhou C-F, Wings O, Ge S, Joyce WG. 2013. A new xinjiangchelyid turtle from the

2321 Middle Jurassic of Xinjiang, China and the evolution of the basipterygoid process in

2322 Mesozoic turtles. BMC Evolutionary Biology 13:203.

2323 Revell LJ. 2010. Phylogenetic signal and linear regression on species data. Methods in Ecology 2324 and Evolution 1:319-329.

2325

2326

2327

2328

2329

2330

2331

2332

2333

2334

2335

2336

2337

Rohlf FJ. 2001. Comparative methods for the analysis of continuous variables: geometric interpretations. Evolution 55:2143-2160.

Rollot Y, Lyson TR, Joyce WG. 2018. A description of the skull of Eubaena cephalica (Hay, 1904) and new insights into the cranial circulation and innervation of baenid turtles. Journal of Vertebrate Paleontology e1474886.

Ruge G. 1987. Über das peripherische Gebiet des Nervus facialis bei Wirbeltieren. In: Festschrift zum 70. Geburtstag von C. Gegenbaur. Bd. 3. Leipzig: W. Engelmann, 193-348.

Schumacher G-H. 1973. Die Kopf- und Halsregion der Lederschildkröte Dermochelys coriacea (Linnaeus 1766). Abhandlungen der Akademie der Wissenschaften der DDR 2:1-60.

Sedlmayr JC. 2002. Anatomy, evolution, and functional significance of cephalic vasculature in Archosauria. PhD Thesis, Ohio University.

Shaffer HB, Meylan P, McKnight ML, Larson A. 1997. Tests of turtle phylogeny: molecular, morphological, and paleontological approaches. Systematic Biology 46:235-268. 
2338 Shiino K. 1913. Beitrag zur Kenntnis der Gehimnerven der Schildkroten. Anatomische Hefte 47:1-34.

Shindo T. 1914. Zur vergleichenden Anatomie der arteriellen Kopfgefässe der Reptilien. Anatomische Hefte 51:267-356.

Shishkin MA. 1968. On the cranial arterial system of the labyrinthodonts. Acta Zoologica 49:122.

Siebenrock F. 1897. Das Kopfskelet der Schildkröten. Sitzungsberichte der Kaiserlichen Akademie der Wissenschaften 106:245-328.

Smaers JB, Mongle CS. 2018. Evomap: R package for the evolutionary mapping of continuous traits. San Francisco: Github.

Smaers JB, Rohlf FJ. 2016. Testing species' deviation from allometric predictions using the phylogenetic regression. Evolution 70:1145-1149.

Soliman MA. 1964. Die Kopfnerven der Schildkröten. Zeitschrift für Wissenschaftliche Zoologie 169:216-312.

Starck D. 1979. Cranio-cerebral relations in recent reptiles. Biology of the Reptilia 9:1-38.

Sterli J, de la Fuente MS. 2010. Anatomy of Condorchelys antiqua Sterli, 2008, and the origin of the modern jaw closure mechanism in turtles. Journal of Vertebrate Paleontology 30:351366.

Sterli J, Joyce WG. 2007. The cranial anatomy of the Early Jurassic turtle Kayentachelys aprix. Acta Palaeontologica Polonica 52:675-694.

Sterli J, de la Fuente MS. 2013. New evidence from the Palaeocene of Patagonia (Argentina) on the evolution and palaeo-biogeography of Meiolaniformes (Testudinata, new taxon name). Journal of Systematic Palaeontology 11:835-852. 
2361 Sterli J, Müller J, Anquetin J, Hilger A. 2010. The parabasisphenoid complex in Mesozoic

2362 turtles and the evolution of the testudinate basicranium. Canadian Journal of Earth Sciences

$2363 \quad 47: 1337-1346$.

2364 Sukhanov VB. 2000. Mesozoic turtles of Middle and Central Asia. In: Benton MJ, Shishkin

2365 MA, Unwin DM, Kurochkin EN, eds. The age of dinosaurs in Russia and Mongolia.

2366 Cambridge: Cambridge University Press, 309-367.

2367 Symonds MRE, Blomberg SP. 2014. A primer on phylogenetic generalised least squares. A

2368 primer on phylogenetic generalised least squares. In: Garamszegi L, eds. Modern

2369 phylogenetic comparative methods and their application in evolutionary biology. Berlin,

2370 Heidelberg: Springer, 105-130.

2371 van der Merwe NJ. 1940. Die Skedelmorfologie van Pelomedusa galeata (Wagler). Tydskrif vir

2372 Wetenskap en Kuns 1:67-85.

2373 Vogt, C. 1840. Beiträge zur Neurologie der Reptilien. Neue Denkschrift der Allgemeinen

2374 Schweitzerischen Gesellschaft für die gesammten Naturwissenschaften 4:40-55.

2375

2376

2377

2378

2379

2380

2381

2382

2383

Yu E, Ashwell KWS, Shulruf B. 2019 (preprint). Quantitative analysis of arterial supply to the developing brain in tetrapod vertebrates. Anatomical Record.

Zangerl R. 1953. The vertebrate fauna of the Selma Formation of Alabama. Part IV. The turtles of the family Toxochelyidae. Fieldiana: Geology Memoirs 3:135-277.

Zhou C-F, Rabi M. 2015. A sinemydid turtle from the Jehol Biota provides insights into the basal divergence of crown turtles. Scientific Reports 5:16299.

\section{Figure 1. Basic scheme of internal carotid artery, lateral head vein, and facial nerve} systems in turtles. 
2385

2386

2387

2388

2389

2390

2391

2392

2393

2394

2395

2396

2397

2398

2399

2400

2401

2402

2403

2404

2405

2406

Figure 2. Simplified phylogenetic tree showing the relationships between crown clades turtles, following Crawford et al. (2015).

\section{Figure 3. The carotid circulation and vidian canal system of Chelus fimbriatus (NHMUK}

81.9.27.4). Three-dimensional reconstructions of the basisphenoid, right pterygoid, in (A) dorsal, (B) ventral, and (C) left lateral view. Illustration in dorsal view (D) highlighting the placement of relevant arteries, nerves, and veins. Dark colors highlight sections fully covered by bone, light colors partially or fully uncovered sections. Abbreviations: ac, arteria carotis cerebralis; aci, arteria carotis interna; bs, basisphenoid; 'cc', secondary canalis cavernosus; $\mathbf{c c b}$, canalis caroticus basisphenoidalis; cci, canalis caroticus internus; ccv, canalis cavernosus; cnf, canalis nervus facialis; cnhp, canalis nervus hyomandibularis proximalis; cnv, canalis nervus vidianus; faccb, foramen anterius canalis carotici basisphenoidalis; facnv, foramen anterius canalis nervi vidiani; fpcci, foramen posterius canalis carotici interni; gg, geniculate ganglion; pt, pterygoid; vcl, vena capitis lateralis; VII, nervus facialis; $\mathbf{V I I}_{\mathbf{h y}}$, nervus hyomandibularis; $\mathbf{V I I}_{\mathbf{v i}}$, nervus vidiani.

\section{Figure 4. The carotid circulation and vidian canal system of Podocnemis unifilis (NHMUK}

60.4.16.9). Three-dimensional reconstructions of the basisphenoid, right pterygoid, in (A) dorsal, (B) ventral, and (C) left lateral view. Illustration in dorsal view (D) highlighting the placement of relevant arteries, nerves, and veins. Dark colors highlight sections fully covered by bone, light colors partially or fully uncovered sections. Abbreviations: ac, arteria carotis cerebralis; aci, arteria carotis interna; bs, basisphenoid; ccb, canalis caroticus basisphenoidalis; ccv, canalis 
2407 cavernosus; cnf, canalis nervus facialis; cnhd, canalis nervus hyomandibularis distalis; $\mathbf{c n h p ,}$

2408 canalis nervus hyomandibularis proximalis; cnv, canalis nervus vidianus; cprnv, canalis pro

2409 ramo nervi vidiani; faccb, foramen anterius canalis carotici basisphenoidalis; facnv, foramen

2410 anterius canalis nervi vidiani; gg, geniculate ganglion; pt, pterygoid; vcl, vena capitis lateralis;

2411 VII, nervus facialis; $\mathbf{V I I}_{\mathbf{h y}}$, nervus hyomandibularis; $\mathbf{V I I}_{\mathbf{v i}}$, nervus vidiani.

Figure 5. The carotid circulation and vidian canal system of Pelusios subniger (NMB

16230). Three-dimensional reconstructions of the basisphenoid, right pterygoid, in (A) dorsal,

(B) ventral, and (C) left lateral view. Illustration in dorsal view (D) highlighting the placement of relevant arteries, nerves, and veins. Dark colors highlight sections fully covered by bone, light colors partially or fully uncovered sections. Abbreviations: ac, arteria carotis cerebralis; aci, arteria carotis interna; bs, basisphenoid; ccb, canalis caroticus basisphenoidalis; $\mathbf{c n h}$, canalis nervus hyomandibularis; cci, canalis caroticus internus; ccv, canalis cavernosus; cnf, canalis nervus facialis; cnhp, canalis nervus hyomandibularis proximalis; cnv, canalis nervus vidianus; faccb, foramen anterius canalis carotici basisphenoidalis; facnv, foramen anterius canalis nervi vidiani; fpcci, foramen posterius canalis carotici interni; gg, geniculate ganglion; pt, pterygoid; vcl, vena capitis lateralis; VII, nervus facialis; $\mathbf{V I I}_{\mathbf{h y}}$, nervus hyomandibularis; $\mathbf{V I I}_{\mathbf{v i}}$, nervus vidiani.

\section{Figure 6. The carotid circulation and vidian canal system of Carettochelys insculpta}

(NHMUK 1903.7.10.1). Three-dimensional reconstructions of the basisphenoid, right pterygoid, in (A) dorsal, (B) ventral, and (C) left lateral view. Illustration in dorsal view (D) highlighting the placement of relevant arteries, nerves, and veins. Dark colors highlight sections fully covered 
2430 by bone, light colors partially or fully uncovered sections. The red portion of the canalis

2431 cavernosus shows the inferred position of the mandibular artery. Abbreviations: ac, arteria

2432 carotis cerebralis; aci, arteria carotis interna; am, arteria mandibularis; bs, basisphenoid; ccb,

2433 canalis caroticus basisphenoidalis; cci, canalis caroticus internus; ccv, canalis cavernosus; cnf,

2434 canalis nervus facialis; cnv, canalis nervus vidianus; cprnv, canalis pro ramo nervi vidiani;

2435 faccb, foramen anterius canalis carotici basisphenoidalis; facnv, foramen anterius canalis nervi

2436 vidiani; fpcci, foramen posterius canalis carotici interni; gg, geniculate ganglion; pt, pterygoid;

2437 vcl, vena capitis lateralis; VII, nervus facialis; $\mathbf{V I I}_{\mathbf{h y}}$, nervus hyomandibularis; $\mathbf{V I I}_{\mathbf{v i}}$, nervus

2438 vidiani.

2439

2440

Figure 7. The carotid circulation and vidian canal system of Apalone spinifera (FMNH

2441

22178). Three-dimensional reconstructions of the basisphenoid, right pterygoid, in (A) dorsal,

2442

(B) ventral, and (C) left lateral view. Illustration in dorsal view (D) highlighting the placement of

2443

relevant arteries, nerves, and veins. Dark colors highlight sections fully covered by bone, light

2444

colors partially or fully uncovered sections. Abbreviations: ac, arteria carotis cerebralis; aci,

2445 arteria carotis interna; am, arteria mandibularis; bs, basisphenoid; ccb, canalis caroticus

2446 basisphenoidalis; cci, canalis caroticus internus; ccl, canalis caroticus lateralis; ccv, canalis

2447 cavernosus; $\mathbf{c n f}$, canalis nervus facialis; cnv, canalis nervus vidianus; faccb, foramen anterius

2448 canalis carotici basisphenoidalis; faccl, foramen anterius canalis caroticus lateralis; facnv,

2449 foramen anterius canalis nervi vidiani; fpcci, foramen posterius canalis carotici interni; gg,

2450 geniculate ganglion; pt, pterygoid; vcl, vena capitis lateralis; VII, nervus facialis; $\mathbf{V I I}_{\text {hy }}$, nervus

2451 hyomandibularis; $\mathbf{V I I}_{\mathbf{v i}}$, nervus vidiani.

2452

Peer] reviewing PDF | (2020:05:49210:2:0:NEW 10 Nov 2020) 
2453 Figure 8. The carotid circulation and vidian canal system of Sternotherus minor (FMNH

2454 211696). Three-dimensional reconstructions of the basisphenoid, right pterygoid, in (A) dorsal,

2455 (B) ventral, and (C) left lateral view. Illustration in dorsal view (D) highlighting the placement of 2456 relevant arteries, nerves, and veins. Dark colors highlight sections fully covered by bone, light

2457 colors partially or fully uncovered sections. Abbreviations: ac, arteria carotis cerebralis; aci,

2458 arteria carotis interna; ap, arteria palatina; am, arteria mandibularis; bs, basisphenoid; ccb,

2459 canalis caroticus basisphenoidalis; cci, canalis caroticus internus; ccl, canalis caroticus lateralis;

2460 ccv, canalis cavernosus; cnf, canalis nervus facialis; cnv, canalis nervus vidianus; cprnv, canalis

2461 pro ramo nervi vidiani; faccb, foramen anterius canalis carotici basisphenoidalis; faccl, foramen

2462 anterius canalis caroticus lateralis; facnv, foramen anterius canalis nervi vidiani; fpcci, foramen

2463 posterius canalis carotici interni; gg, geniculate ganglion; pt, pterygoid; vcl, vena capitis

2464 lateralis; VII, nervus facialis; $\mathbf{V I I}_{\mathbf{h y}}$, nervus hyomandibularis; $\mathbf{V I I}_{\mathbf{v i}}$, nervus vidiani.

2465

2466

Figure 9. The carotid circulation and vidian canal system of Dermatemys mawii (SMF

2467 59463). Three-dimensional reconstructions of the basisphenoid, right pterygoid, in (A) dorsal,

2468 (B) ventral, and (C) left lateral view. Illustration in dorsal view (D) highlighting the placement of

2469 relevant arteries, nerves, and veins. Dark colors highlight sections fully covered by bone, light

2470 colors partially or fully uncovered sections. The red portion of the canalis cavernosus shows the

2471 inferred position of the mandibular artery. Abbreviations: ac, arteria carotis cerebralis; aci,

2472 arteria carotis interna; am-a, anterior arteria mandibularis; am-p, posterior arteria mandibularis;

2473 ap, arteria palatina; bs, basisphenoid; ccb, canalis caroticus basisphenoidalis; cci, canalis

2474 caroticus internus; ccl, canalis caroticus lateralis; ccv, canalis cavernosus; cnf, canalis nervus

2475 facialis; cnv, canalis nervus vidianus; faccb, foramen anterius canalis carotici basisphenoidalis; 
2476 faccl, foramen anterius canalis caroticus lateralis; facnv, foramen anterius canalis nervi vidiani;

2477 fpcci, foramen posterius canalis carotici interni; gg, geniculate ganglion; pt, pterygoid; vcl, vena

2478 capitis lateralis; VII, nervus facialis; $\mathbf{V I I}_{\mathbf{h y}}$, nervus hyomandibularis; $\mathbf{V I I}_{\mathbf{v i}}$, nervus vidiani.

Figure 10. The carotid circulation and vidian canal system of Chelydra serpentina (SMF

32846). Three-dimensional reconstructions of the basisphenoid, right pterygoid, in (A) dorsal,

(B) ventral, and (C) left lateral view. Illustration in dorsal view (D) highlighting the placement of relevant arteries, nerves, and veins. Dark colors highlight sections fully covered by bone, light colors partially or fully uncovered sections. The red portion of the canalis cavernosus shows the inferred position of the mandibular artery. Abbreviations: ac, arteria carotis cerebralis; aci, arteria carotis interna; ap, arteria palatina; am, arteria mandibularis; bs, basisphenoid; ccb, canalis caroticus basisphenoidalis; cci, canalis caroticus internus; ccl, canalis caroticus lateralis; ccv, canalis cavernosus; cnf, canalis nervus facialis; cnv, canalis nervus vidianus; faccb, foramen anterius canalis carotici basisphenoidalis; faccl, foramen anterius canalis caroticus lateralis; facnv, foramen anterius canalis nervi vidiani; fpcci, foramen posterius canalis carotici interni; gg, geniculate ganglion; pt, pterygoid; vcl, vena capitis lateralis; VII, nervus facialis; VII $_{\text {hy }}$, nervus hyomandibularis; $\mathbf{V I I}$, , nervus vidiani. (FMNH 22242). Three-dimensional reconstructions of the basisphenoid, right pterygoid, in (A) dorsal, (B) ventral, and (C) left lateral view. Illustration in dorsal view (D) highlighting the placement of relevant arteries, nerves, and veins. Dark colors highlight sections fully covered by bone, light colors partially or fully uncovered sections. The red portion of the canalis cavernosus 
2499 shows the inferred position of the posterior mandibular artery. Abbreviations: ac, arteria carotis

2500 cerebralis; aci, arteria carotis interna; ap, arteria palatina; am-a, anterior arteria mandibularis;

2501 am-p, posterior arteria mandibularis; bs, basisphenoid; ccb, canalis caroticus basisphenoidalis;

2502 cci, canalis caroticus internus; ccv, canalis cavernosus; cnf, canalis nervus facialis; cprnv,

2503 canalis pro ramo nervi vidiani; faccb, foramen anterius canalis carotici basisphenoidalis; fpcci,

2504 foramen posterius canalis carotici interni; gg, geniculate ganglion; pt, pterygoid; v-am, vestigial

2505 arteria mandibularis; vcl, vena capitis lateralis; VII, nervus facialis; $\mathbf{V I I}_{\mathbf{h y}}$, nervus

2506 hyomandibularis; $\mathbf{V I I}_{\mathbf{v i}}$, nervus vidiani.

2507

2508

Figure 12. The carotid circulation and vidian canal system of Dermochelys coriacea (FMNH

2509

171756). Three-dimensional reconstructions of the basisphenoid, right pterygoid, in (A) dorsal,

2510

(B) ventral, and (C) left lateral view. Illustration in dorsal view (D) highlighting the placement of

2511

relevant arteries, nerves, and veins. Dark colors highlight sections fully covered by bone, light

2512

colors partially or fully uncovered sections. Abbreviations: ac, arteria carotis cerebralis; aci,

2513

arteria carotis interna; am, arteria mandibularis; ap, arteria palatina; bs, basisphenoid; cci,

2514

canalis caroticus internus; ccv, canalis cavernosus; cnf, canalis nervus facialis; pt, pterygoid; vcl,

2515

vena capitis lateralis; VII, nervus facialis; $\mathbf{V I I}_{\mathbf{h y}}$, nervus hyomandibularis; $\mathbf{V I I}_{\mathbf{v i}}$, nervus vidiani.

2516

Note that we do not highlight the course of the mandibular artery, because the mandibular blood

2517 supply of Dermochelys coriacea is currently unknown.

2518

2519

Figure 13. The carotid circulation and vidian canal system of Platysternon megacephalum

2520

(SMF 69684). Three-dimensional reconstructions of the basisphenoid, right pterygoid, in (A)

2521 dorsal, (B) ventral, and (C) left lateral view. Illustration in dorsal view (D) highlighting the 
2522 placement of relevant arteries, nerves, and veins. Dark colors highlight sections fully covered by

2523 bone, light colors partially or fully uncovered sections. The red portion of the canalis cavernosus

2524 shows the inferred position of the mandibular artery. Abbreviations: ac, arteria carotis cerebralis;

2525 aci, arteria carotis interna; am, arteria mandibularis; bs, basisphenoid; ccb, canalis caroticus

2526 basisphenoidalis; cci, canalis caroticus internus; ccv, canalis cavernosus; cnf, canalis nervus

2527 facialis; cnv, canalis nervus vidianus; cprnv, canalis pro ramo nervi vidiani; faccb, foramen

2528 anterius canalis carotici basisphenoidalis; facnv, foramen anterius canalis nervi vidiani; fpcci,

2529 foramen posterius canalis carotici interni; gg, geniculate ganglion; pt, pterygoid; vcl, vena capitis

2530 lateralis; VII, nervus facialis; $\mathbf{V I I}_{\mathbf{h y}}$, nervus hyomandibularis; $\mathbf{V I I}_{\mathbf{v i}}$, nervus vidiani.

2531

2532

Figure 14. The carotid circulation and vidian canal system of Glyptemys insculpta (FMNH

2533

22240). Three-dimensional reconstructions of the basisphenoid, right pterygoid, in (A) dorsal,

2534

(B) ventral, and (C) left lateral view. Illustration in dorsal view (D) highlighting the placement of

2535

relevant arteries, nerves, and veins. Dark colors highlight sections fully covered by bone, light

2536

colors partially or fully uncovered sections. The red portion of the canalis cavernosus shows the

2537

inferred position of the mandibular artery. Abbreviations: ac, arteria carotis cerebralis; aci,

2538

arteria carotis interna; ap, arteria palatina; am, arteria mandibularis; bs, basisphenoid; ccb,

2539

canalis caroticus basisphenoidalis; cci, canalis caroticus internus; ccl, canalis caroticus lateralis;

2540

ccv, canalis cavernosus; cnf, canalis nervus facialis; cnv, canalis nervus vidianus; cprnv, canalis

2541

pro ramo nervi vidiani; faccb, foramen anterius canalis carotici basisphenoidalis; faccl, foramen

2542

anterius canalis caroticus lateralis; facnv, foramen anterius canalis nervi vidiani; fpcci, foramen

2543

posterius canalis carotici interni; gg, geniculate ganglion; pt, pterygoid; vcl, vena capitis

2544 lateralis; VII, nervus facialis; $\mathbf{V I I}_{\mathbf{h y}}$, nervus hyomandibularis; $\mathbf{V I I}_{\mathbf{v i}}$, nervus vidiani. 
2546 Figure 15. The carotid circulation and vidian canal system of Gopherus agassizii (FMNH

2547 216746). Three-dimensional reconstructions of the basisphenoid, right pterygoid, in (A) dorsal,

2548 (B) ventral, and (C) left lateral view. Illustration in dorsal view (D) highlighting the placement of

2549 relevant arteries, nerves, and veins. Dark colors highlight sections fully covered by bone, light

2550 colors partially or fully uncovered sections. The red portion of the canalis cavernosus shows the

2551 inferred position of the mandibular artery. Abbreviations: ac, arteria carotis cerebralis; aci,

2552 arteria carotis interna; am, arteria mandibularis; bs, basisphenoid; ccb, canalis caroticus

2553 basisphenoidalis; cci, canalis caroticus internus; ccv, canalis cavernosus; cnf, canalis nervus

2554 facialis; cnv, canalis nervus vidianus; faccb, foramen anterius canalis carotici basisphenoidalis;

2555 facnv, foramen anterius canalis nervi vidiani; fam, foramen arteriomandibulare; fpcci, foramen

2556 posterius canalis carotici interni; gg, geniculate ganglion; pt, pterygoid; vcl, vena capitis

2557 lateralis; VII, nervus facialis; $\mathbf{V I I}_{\mathbf{h y}}$, nervus hyomandibularis; $\mathbf{V I I}_{\mathbf{v i}}$, nervus vidiani.

2558

2559 Figure 16. The carotid circulation and vidian canal system of Batagur baska (NHMUK

2560 67.9.28.7). Three-dimensional reconstructions of the basisphenoid, right pterygoid, in (A) dorsal,

2561 (B) ventral, and (C) left lateral view. Illustration in dorsal view (D) highlighting the placement of

2562 relevant arteries, nerves, and veins. Dark colors highlight sections fully covered by bone, light

2563 colors partially or fully uncovered sections. The red portion of the canalis cavernosus shows the

2564 inferred position of the mandibular artery. Abbreviations: ac, arteria carotis cerebralis; aci,

2565 arteria carotis interna; ap, arteria palatina; am, arteria mandibularis; bs, basisphenoid; ccb,

2566 canalis caroticus basisphenoidalis; cci, canalis caroticus internus; ccl, canalis caroticus lateralis;

2567 cev, canalis cavernosus; cnf, canalis nervus facialis; cnv, canalis nervi vidiani; cprnv, canalis 
2568 pro ramo nervi vidiani; faccb, foramen anterius canalis caroticus cerebralis; faccl, foramen

2569 anterius canalis caroticus lateralis; facnv, foramen anterius canalis nervi vidiani; fpcci, foramen

2570 posterius canalis carotici interni; gg, geniculate ganglion; pt, pterygoid; vcl, vena capitis

2571 lateralis; VII, nervus facialis; $\mathbf{V I I}_{\mathbf{h y}}$, nervus hyomandibularis; $\mathbf{V I I}_{\mathbf{v i}}$, nervus vidiani.

2572

2573

2574

Figure 17. Asymmetry in osteological correlates for the palatine artery in Manouria

impressa (SMF 69777). (A), dorsal view of horizontally cut basicranium for orientation. (B), as

2575

A, but zoomed in on details of anterior exiting foramina for the carotid arterial system. (C),

2576

cranium in left lateral view, showing position of axial slices shown in D-E. (D), axial CT slice at

2577

position of foramina anterius canalis carotici basisphenoidalis. (E), axial CT slice at position of

2578

carotid split. Note that canals and foramina for the palatine artery are present on the right side,

2579

although the palatine artery is generally absent in testudinids. Abbreviations: bo, blind opening

2580

(opens into bone, but does not connect to blood or nervous system); ccb, canalis caroticus

2581

basisphenoidalis; faccb, foramen anterius canalis carotici basisphenoidalis; faccl, foramen

2582

anterius canalis carotici lateralis; $\mathbf{r}-\mathbf{c c l}$, right canalis caroticus lateralis; $\mathbf{t c b}$, trabeculae of

2583

cancellous bone (small internal openings not connect to blood or nervous system).

2584

2585

2586

Figure 18. Schematic overview of patterns pertaining to the split of the facial nerve into its hyomandibular and vidian branches. Abbreviations: caj, cavum acustico-jugulare; ccv, canalis

2587

cavernosus; cci, canalis caroticus internus; ccl, canalis caroticus lateralis; cnf, canalis nervus

2588

facialis; cnhp, canalis nervus hyomandibularis proximalis; cnv, canalis nervus vidianus; cprnv,

2589

canalis pro ramo nervi vidiani; faf, fossa acustico-facialis; fpcci, foramen posterius canalis 
2590 carotici interni; gg, geniculate ganglion; VII, nervus facialis; $\mathbf{V I I}_{\mathrm{hy}}$, nervus hyomandibularis;

2591 VII $_{\mathrm{vi}}$, nervus vidiani.

2592

2593

Figure 19. Schematic overview of patterns pertaining to the posterior portion of the

2594

hyomandibular nerve. The five patterns presented herein only apply to taxa in which the vidian

2595

nerve enters the carotid canal system (i.e. turtles with patterns IA, IIA, and IIB). Abbreviations:

2596

caj, cavum acustico-jugulare; ccv, canalis cavernosus; cnhd, canalis nervus hyomandibularis

2597

distalis; snh, sulcus nervus hyomandibularis; $\mathbf{V I I}_{\mathbf{h y}}$, nervus hyomandibularis.

2598

2599

2600

Figure 20. Schematic overview of patterns pertaining to the anterior portion of the vidian

2601

nerve. Abbreviations: cci, canalis caroticus internus; ccl, canalis caroticus lateralis; cnv, canalis nervus vidianus; fpcci, foramen posterius canalis carotici interni; scc, "short cut canal"; scv,

2602 sulcus cavernosus; $\mathbf{V I I}_{\mathbf{v i}}$, vidian nerve.

2603

2604

Figure 21. Relationship of internal carotid artery size and stapedial artery size in turtles.

2605

(A), pGLS regression of $\log _{10^{-}}$cross-sectional diameter of internal carotid artery canal on $\log _{10^{-}}$

2606

cross-sectional diameter of stapedial artery canal. Solid grey line is the regression line describing

2607

a model with a single slope and intercept for all taxa. Dashed grey lines are regression lines for

2608

subsets of the data (see text and table 5 for details). (B), plot showing which data points were

2609

used as subsets for the multiple regression model test. Green points represent the subset

2610

containing kinosternoids, chelonioids, and trionychians, whereas blue points represent the subset

2611

containing the remaining turtle clades. (C), residual plot of pGLS regression of the full dataset,

2612

ordered by clades. Symbols in A and $\mathrm{C}$ denote clade attributions.

Peer] reviewing PDF | (2020:05:49210:2:0:NEW 10 Nov 2020) 
2614 Table 1. List of specimens used in this study. Abbreviations: FMNH, Field Museum of

2615 Natural History, Chicago, IL, USA; NHMUK, Natural History Museum London, London,

2616 England; NMB, Naturhistorisches Museum Basel, Basel, Switzerland; PCHP, Chelonian

2617 Research Institute, Oviedo, FL, USA; PIMUZ, Paläontologisches Museum Zürich, Zürich,

2618 Switzerland; SMF, Senckenberg Naturmuseum Frankfurt, Frankfurt am Main, Germany;

2619 SMNS, Staatliches Museum für Naturkunde Stuttgart, Stuttgart, Germany; UF, Florida Museum

2620 of Natural History, Gainesville, FL, USA; UMZC, University Museum of Zoology Cambridge, 2621 Cambridge, England.

2622

2623 Table 2. Cross-sectional area values $\left(\mu \mathrm{m}^{2}\right)$ of the stapedial and carotid artery canals.

2624 Abbreviations: CCB, canalis caroticus basisphenoidalis; CCI, canalis caroticus internus; CCL, 2625 canalis caroticus lateralis; CST, canalis stapedio-temporalis. Note that value 0 is used when the 2626 canalis caroticus lateralis and arteria palatina are known to be absent, whereas NA (non2627 applicable) is only used for chelonioids, in which the palatine artery is present but does not 2628 extend through its own canal, preventing us from making any measurement of the latter.

Table 3. Comparative table summarizing the main differences between the internal carotid

2631

artery systems. Abbreviations: bs, basisphenoid; CCB, canalis caroticus basisphenoidalis; CCI, canalis caroticus internus; $\mathbf{C C L}$, canalis caroticus lateralis; FPCCI, foramen posterius canalis 2633 carotici interni; int. car. art., internal carotid artery; pal. art., palatine artery; pro, prootic; pt, pterygoid; stap. art., stapedial artery; qu, quadrate. Note that NA (non-applicable) is used for the canalis caroticus lateralis either when the palatine artery is absent (pleurodires, Carettochelys 
2636 insculpta, Platysternon megacephalum, testudinids, and some geoemydids), or because the

2637 palatine artery is not embedded in bone (chelonioids), and for the canalis caroticus

2638 basisphenoidalis as it is not fully ossified in Dermochelys coriacea. Columns of canals and

2639 foramina indicate bones involved in their formation.

2640

2641 Table 4. Comparative table summarizing the main differences between the facial nerve

2642 systems. Abbreviations: bs, basisphenoid; cci, canalis caroticus internus; ccl, canalis caroticus

2643 lateralis; ccv, canalis cavernosus; cnhd, canalis nervus hyomandibularis distalis; cnhp, canalis

2644 nervus hyomandibularis proximalis; cnv, canalis nervus vidianus; conf, hyomandibular nerve

2645 confluent with the canalis cavernosus; epi, epipterygoid; pal, palatine; par, parietal; pro,

2646 prootic; pt, pterygoid; scc, short-cut canal; scv, sulcus cavernosus; sulcus, hyomandibular nerve 2647 contained in a sulcus within the canalis cavernosus. Note that NA (non-applicable) is used when 2648 the canalis nervus vidianus is absent (chelonioids).

2649

2650

Table 5. Results of pGLS regressions of internal carotid artery size on stapedial artery size.

2651 CCI stands for cross-sectional area of the internal carotid artery canal. CST stands for cross-

2652 sectional area of stapedial artery canal. CTK-abbreviated model describes model only including

2653 chelonioids, trionychians, and kinosternoids. Remaining-abbreviated model includes all taxa not

2654 included in the CTK-model. Phylogenetic signal (lambda) was estimated during model fitting. $\mathrm{R}^{2}$

2655 is the generalized coefficient of determination described by Nagelkerke (1991). 
Figure 1

Basic scheme of internal carotid artery, lateral head vein, and facial nerve systems in turtles. 


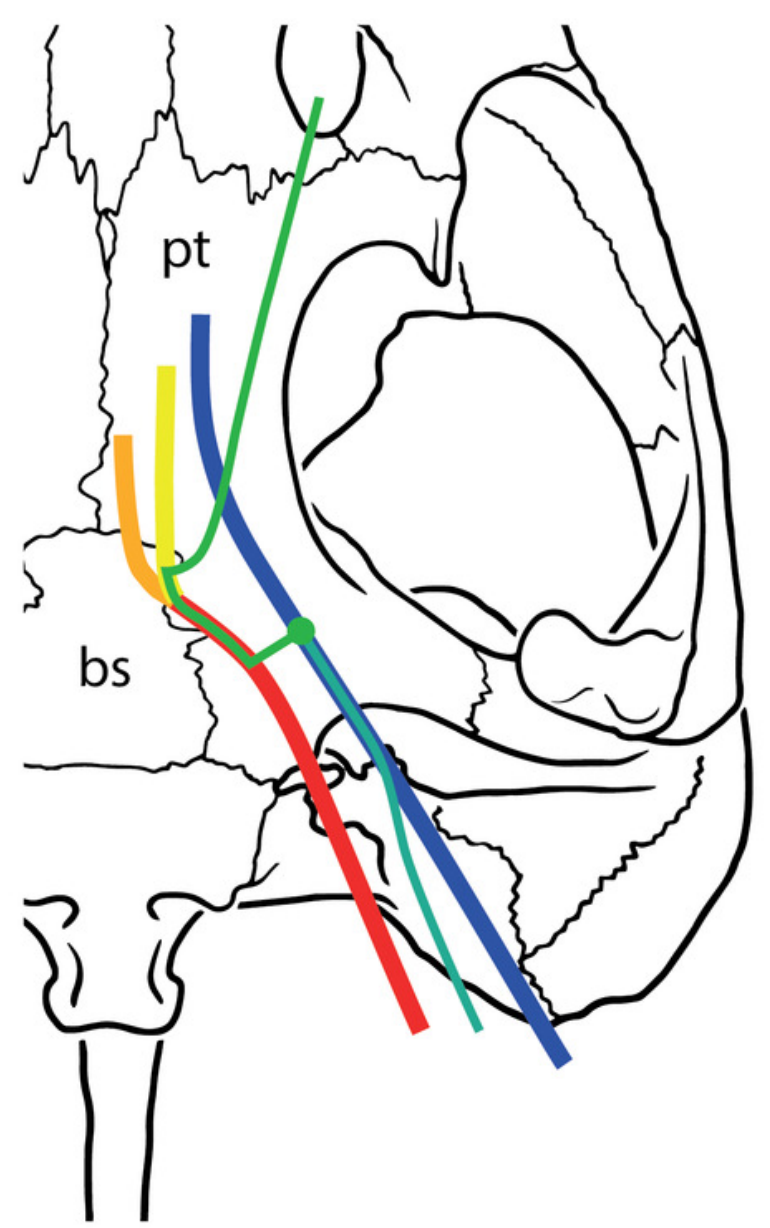

carotid arteries internal branch cerebral branch palatine branch lateral head vein facial nerve (VII) vidian branch hyomandibular branch 
Figure 2

Simplified phylogenetic tree showing the relationships between crown clades turtles, following Crawford et al. (2015).

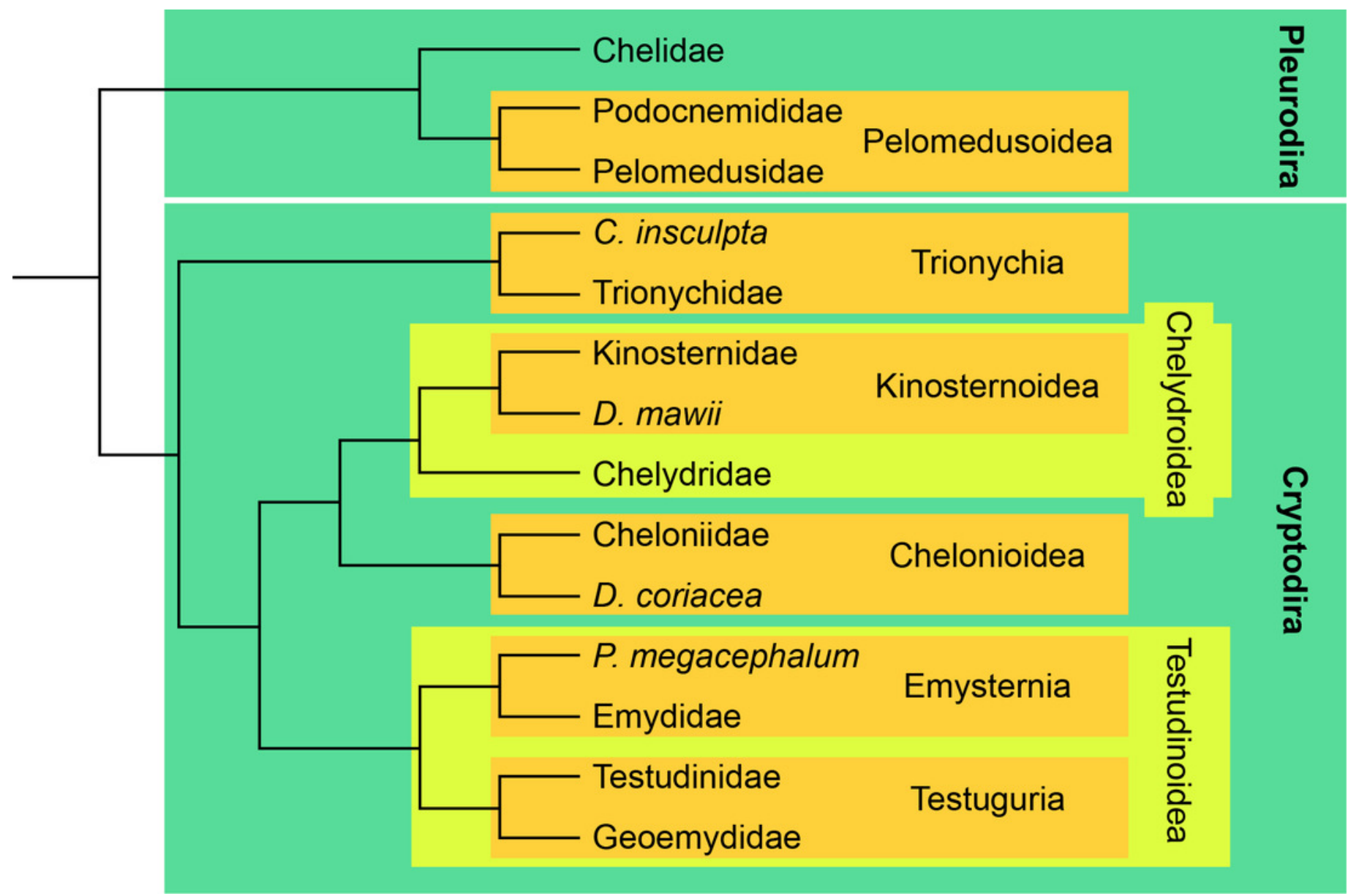




\section{Figure 3}

The carotid circulation and vidian canal system of Chelus fimbriatus (NHMUK 81.9.27.4).

Three-dimensional reconstructions of the basisphenoid, right pterygoid, in (A) dorsal, (B) ventral, and (C) left lateral view. Illustration in dorsal view (D) highlighting the placement of relevant arteries, nerves, and veins. Dark colors highlight sections fully covered by bone, light colors partially or fully uncovered sections. Abbreviations: ac, arteria carotis cerebralis; aci, arteria carotis interna; bs, basisphenoid; 'cc', secondary canalis cavernosus; ccb, canalis caroticus basisphenoidalis; cci, canalis caroticus internus; ccv, canalis cavernosus; cnf, canalis nervus facialis; cnhp, canalis nervus hyomandibularis proximalis; cnv, canalis nervus vidianus; faccb, foramen anterius canalis carotici basisphenoidalis; facnv, foramen anterius canalis nervi vidiani; fpcci, foramen posterius canalis carotici interni; $\mathbf{g g}$, geniculate ganglion; pt, pterygoid; vcl, vena capitis lateralis; VII, nervus facialis; $\mathbf{V I I}_{\mathbf{h y}}$, nervus hyomandibularis; $\mathbf{V I I}_{\mathbf{v i}}$, nervus vidiani. 


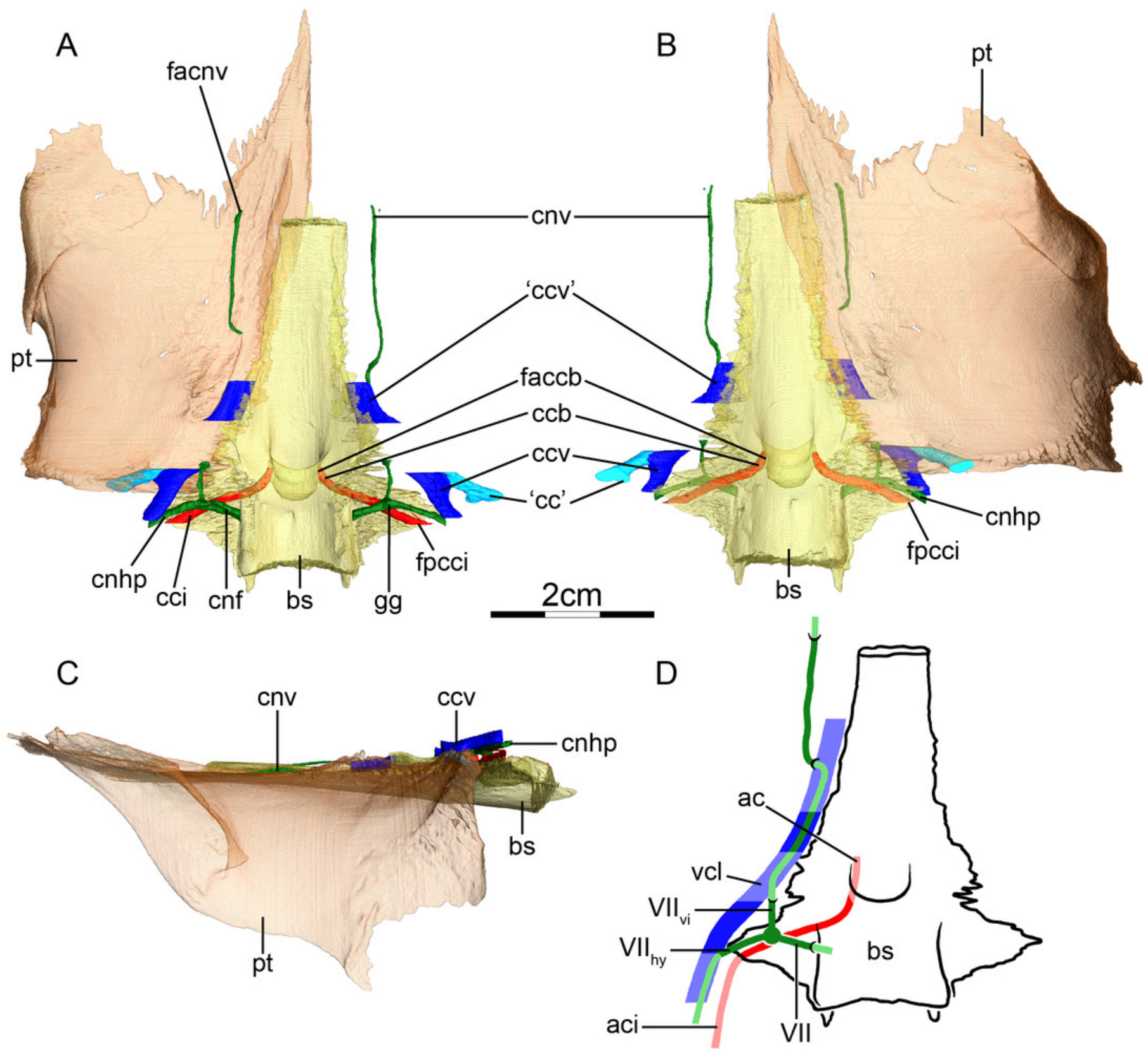




\section{Figure 4}

The carotid circulation and vidian canal system of Podocnemis unifilis (NHMUK 60.4.16.9).

Three-dimensional reconstructions of the basisphenoid, right pterygoid, in (A) dorsal, (B) ventral, and (C) left lateral view. Illustration in dorsal view (D) highlighting the placement of relevant arteries, nerves, and veins. Dark colors highlight sections fully covered by bone, light colors partially or fully uncovered sections. Abbreviations: ac, arteria carotis cerebralis; aci, arteria carotis interna; bs, basisphenoid; $\mathbf{c c b}$, canalis caroticus basisphenoidalis; ccv, canalis cavernosus; cnf, canalis nervus facialis; cnhd, canalis nervus hyomandibularis distalis; cnhp, canalis nervus hyomandibularis proximalis; cnv, canalis nervus vidianus; cprnv, canalis pro ramo nervi vidiani; faccb, foramen anterius canalis carotici basisphenoidalis; facnv, foramen anterius canalis nervi vidiani; gg, geniculate ganglion; pt, pterygoid; vcl, vena capitis lateralis; VII, nervus facialis; $\mathbf{V I I}_{\mathbf{h y}}$, nervus hyomandibularis; $\mathbf{V I I}_{\mathbf{v i}}$, nervus vidiani. 


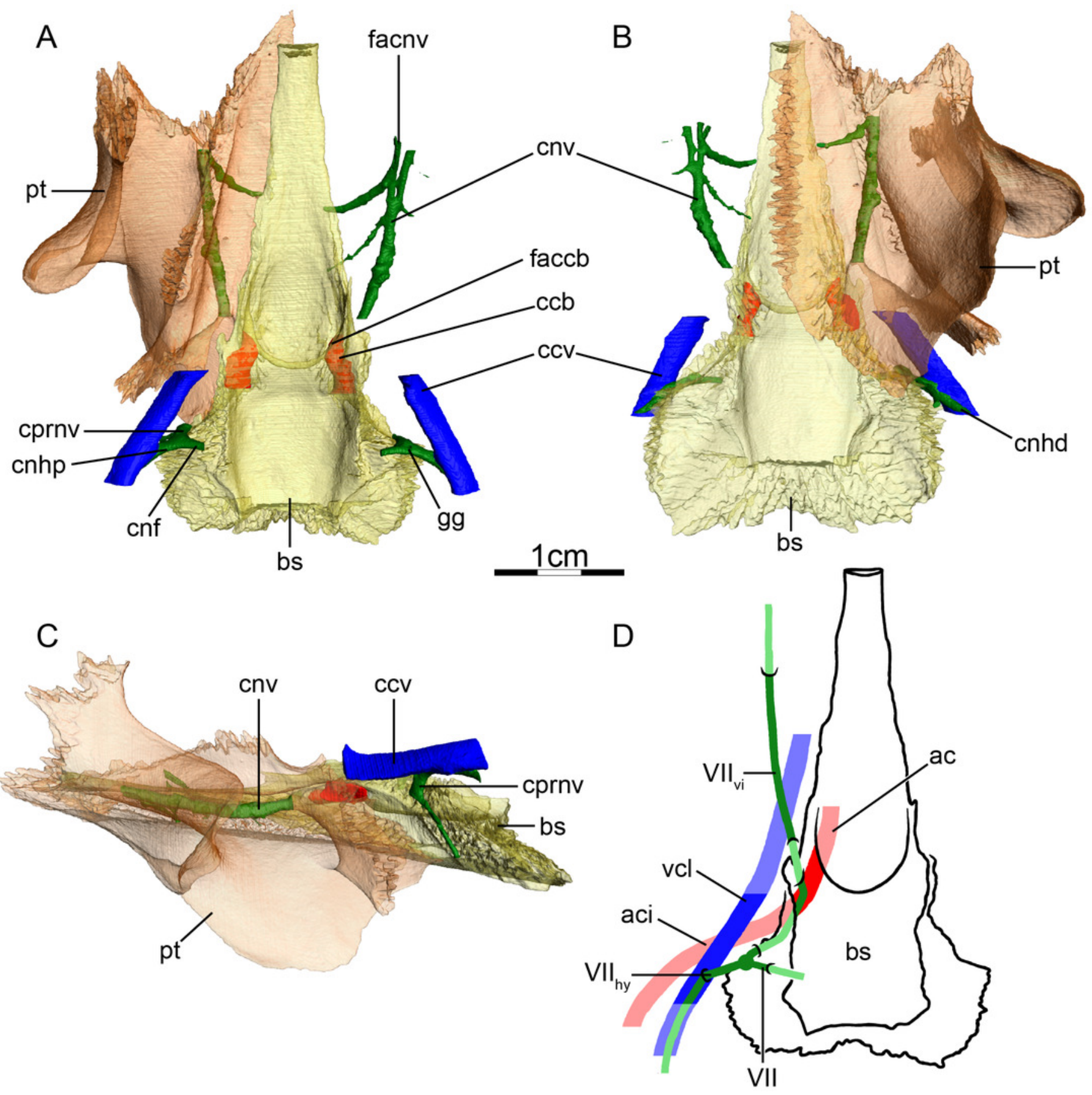




\section{Figure 5}

The carotid circulation and vidian canal system of Pelusios subniger (NMB 16230).

Three-dimensional reconstructions of the basisphenoid, right pterygoid, in (A) dorsal, (B) ventral, and (C) left lateral view. Illustration in dorsal view (D) highlighting the placement of relevant arteries, nerves, and veins. Dark colors highlight sections fully covered by bone, light colors partially or fully uncovered sections. Abbreviations: ac, arteria carotis cerebralis; aci, arteria carotis interna; bs, basisphenoid; $\mathbf{c c b}$, canalis caroticus basisphenoidalis; $\mathbf{c n h}$, canalis nervus hyomandibularis; cci, canalis caroticus internus; ccv, canalis cavernosus; cnf, canalis nervus facialis; $\mathbf{c n h p}$, canalis nervus hyomandibularis proximalis; cnv, canalis nervus vidianus; faccb, foramen anterius canalis carotici basisphenoidalis; facnv, foramen anterius canalis nervi vidiani; fpcci, foramen posterius canalis carotici interni; gg, geniculate ganglion; pt, pterygoid; vcl, vena capitis lateralis; VII, nervus facialis; $\mathbf{V I I}_{\mathbf{h y}}$, nervus hyomandibularis; $\mathbf{V I I}_{\mathbf{v i}}$, nervus vidiani. 


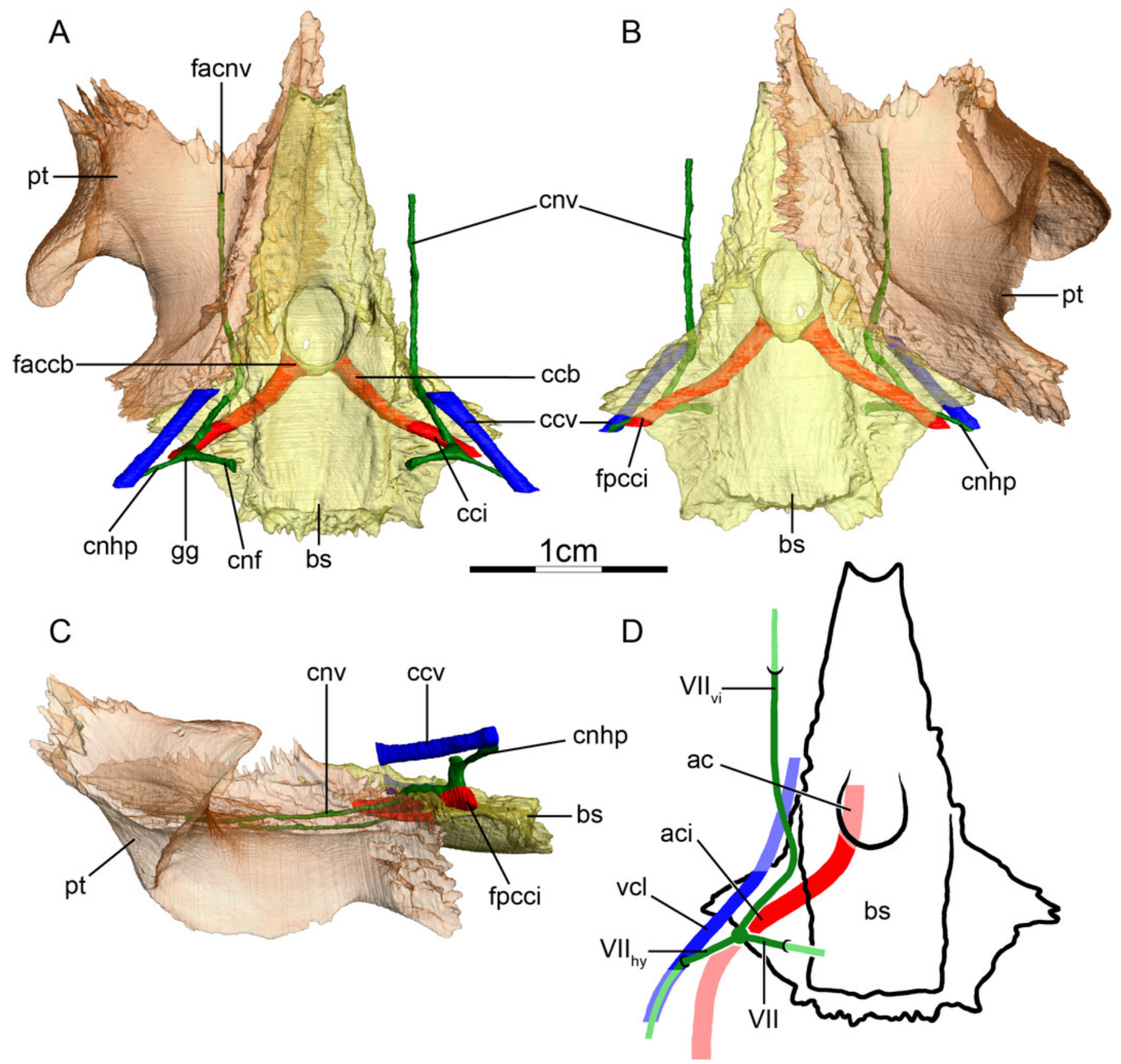




\section{Figure 6}

The carotid circulation and vidian canal system of Carettochelys insculpta (NHMUK 1903.7.10.1).

Three-dimensional reconstructions of the basisphenoid, right pterygoid, in (A) dorsal, (B) ventral, and (C) left lateral view. Illustration in dorsal view (D) highlighting the placement of relevant arteries, nerves, and veins. Dark colors highlight sections fully covered by bone, light colors partially or fully uncovered sections. The red portion of the canalis cavernosus shows the inferred position of the mandibular artery. Abbreviations: ac, arteria carotis cerebralis; aci, arteria carotis interna; am, arteria mandibularis; bs, basisphenoid; ccb, canalis caroticus basisphenoidalis; cci, canalis caroticus internus; ccv, canalis cavernosus; cnf, canalis nervus facialis; cnv, canalis nervus vidianus; cprnv, canalis pro ramo nervi vidiani; faccb, foramen anterius canalis carotici basisphenoidalis; facnv, foramen anterius canalis nervi vidiani; fpcci, foramen posterius canalis carotici interni; $\mathbf{g g}$, geniculate ganglion; pt, pterygoid; vcl, vena capitis lateralis; $\mathbf{V I I}$, nervus facialis; $\mathbf{V I I}_{\mathbf{h y}}$, nervus hyomandibularis; $\mathbf{V I I}_{\mathbf{v i}}$, nervus vidiani. 


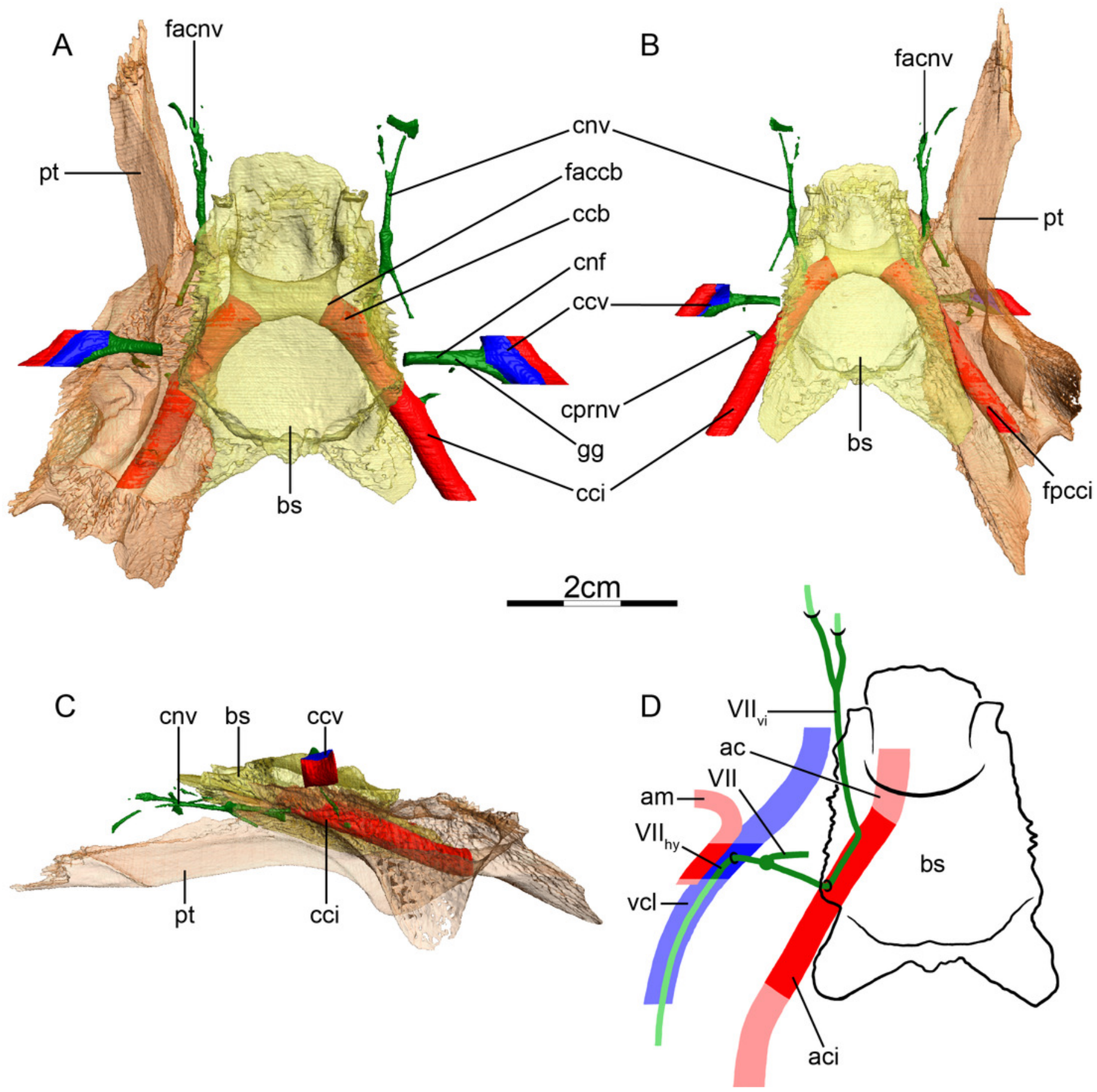




\section{Figure 7}

The carotid circulation and vidian canal system of Apalone spinifera (FMNH 22178).

Three-dimensional reconstructions of the basisphenoid, right pterygoid, in (A) dorsal, (B) ventral, and (C) left lateral view. Illustration in dorsal view (D) highlighting the placement of relevant arteries, nerves, and veins. Dark colors highlight sections fully covered by bone, light colors partially or fully uncovered sections. Abbreviations: ac, arteria carotis cerebralis; aci, arteria carotis interna; am, arteria mandibularis; bs, basisphenoid; $\mathbf{c c b}$, canalis caroticus basisphenoidalis; cci, canalis caroticus internus; ccl, canalis caroticus lateralis; ccv, canalis cavernosus; cnf, canalis nervus facialis; cnv, canalis nervus vidianus; faccb, foramen anterius canalis carotici basisphenoidalis; faccl, foramen anterius canalis caroticus lateralis; facnv, foramen anterius canalis nervi vidiani; fpcci, foramen posterius canalis carotici interni; gg, geniculate ganglion; pt, pterygoid; vcl, vena capitis lateralis; VII, nervus facialis; $\mathbf{V I I}_{\mathbf{h y}}$, nervus hyomandibularis; $\mathbf{V I I}_{\mathbf{v i}}$, nervus vidiani. 


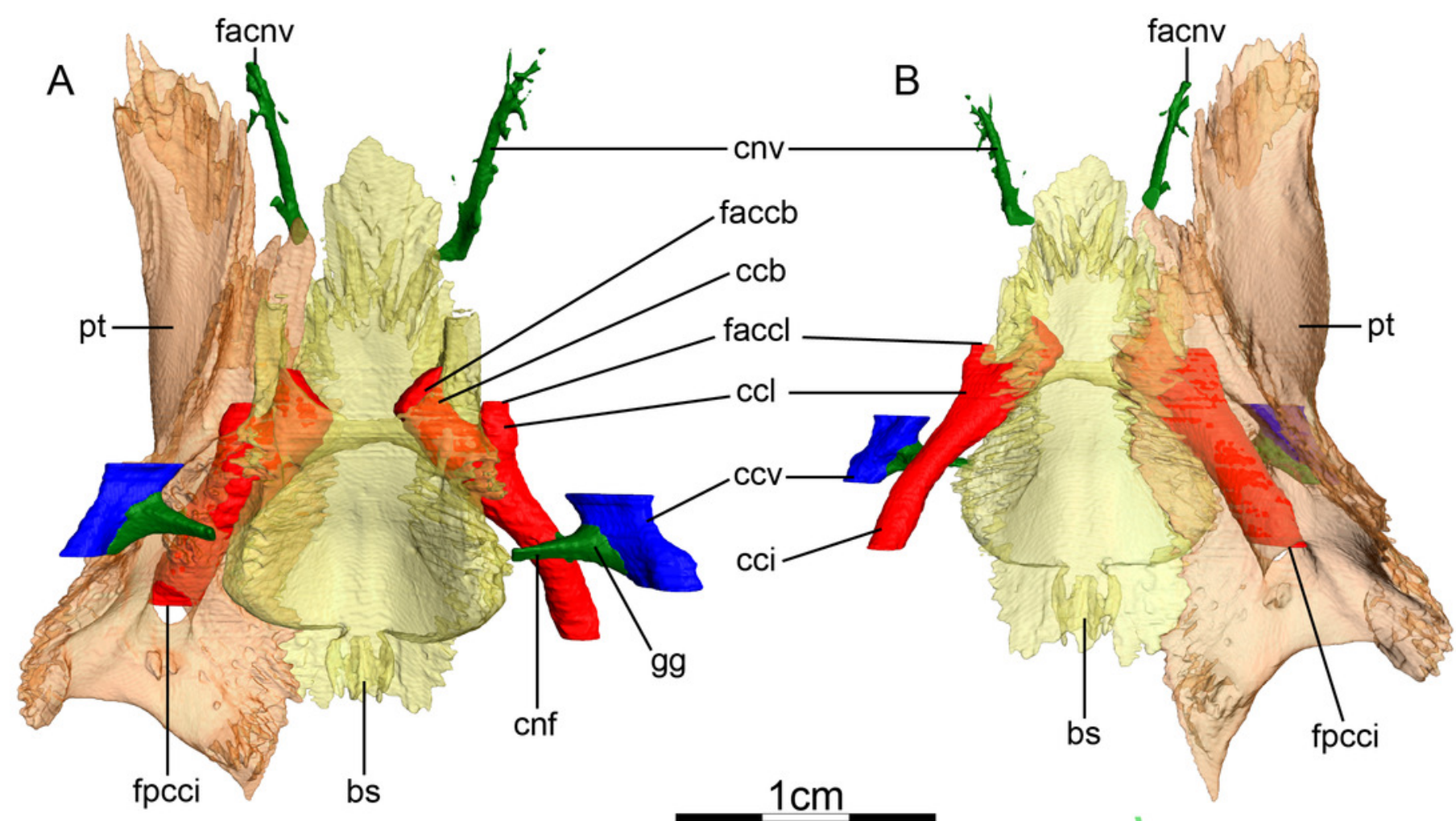

C

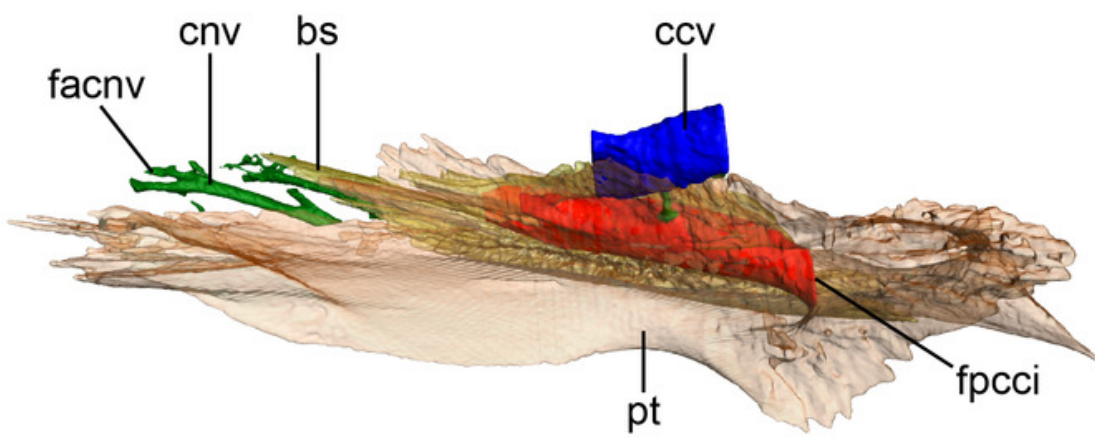

D

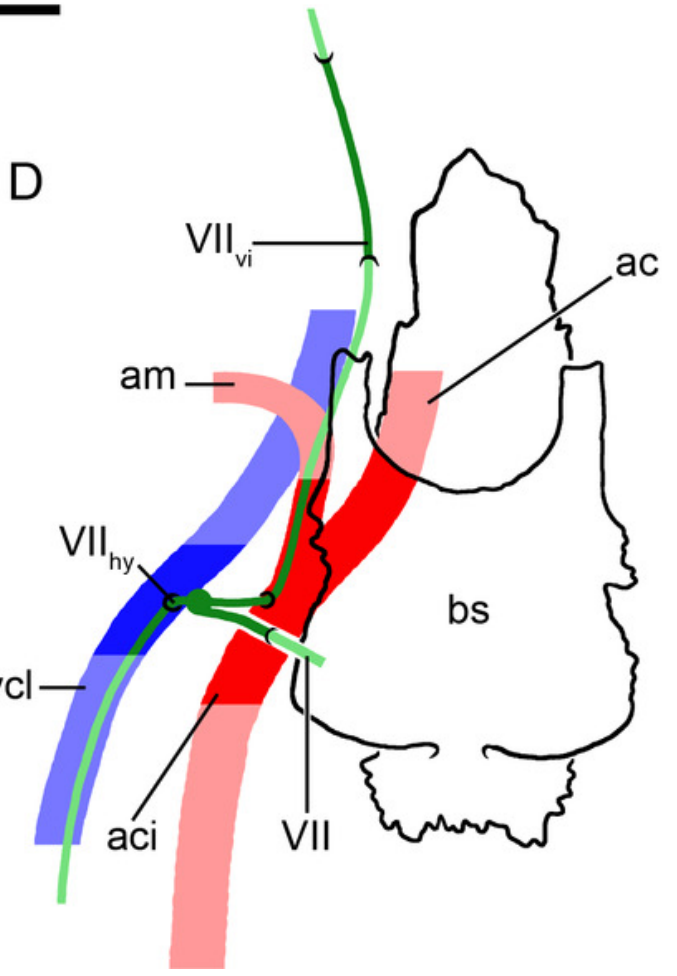




\section{Figure 8}

The carotid circulation and vidian canal system of Sternotherus minor (FMNH 211696)

Three-dimensional reconstructions of the basisphenoid, right pterygoid, in (A) dorsal, (B) ventral, and (C) left lateral view. Illustration in dorsal view (D) highlighting the placement of relevant arteries, nerves, and veins. Dark colors highlight sections fully covered by bone, light colors partially or fully uncovered sections. Abbreviations: ac, arteria carotis cerebralis; aci, arteria carotis interna; ap, arteria palatina; am, arteria mandibularis; bs, basisphenoid; ccb, canalis caroticus basisphenoidalis; cci, canalis caroticus internus; ccl, canalis caroticus lateralis; ccv, canalis cavernosus; cnf, canalis nervus facialis; cnv, canalis nervus vidianus; cprnv, canalis pro ramo nervi vidiani; faccb, foramen anterius canalis carotici basisphenoidalis; faccl, foramen anterius canalis caroticus lateralis; facnv, foramen anterius canalis nervi vidiani; fpcci, foramen posterius canalis carotici interni; gg, geniculate ganglion; pt, pterygoid; vcl, vena capitis lateralis; $\mathbf{V I I}$, nervus facialis; $\mathbf{V I I}_{\mathbf{h y}}$, nervus hyomandibularis; $\mathbf{V I I}_{\mathbf{v i}}$, nervus vidiani. 


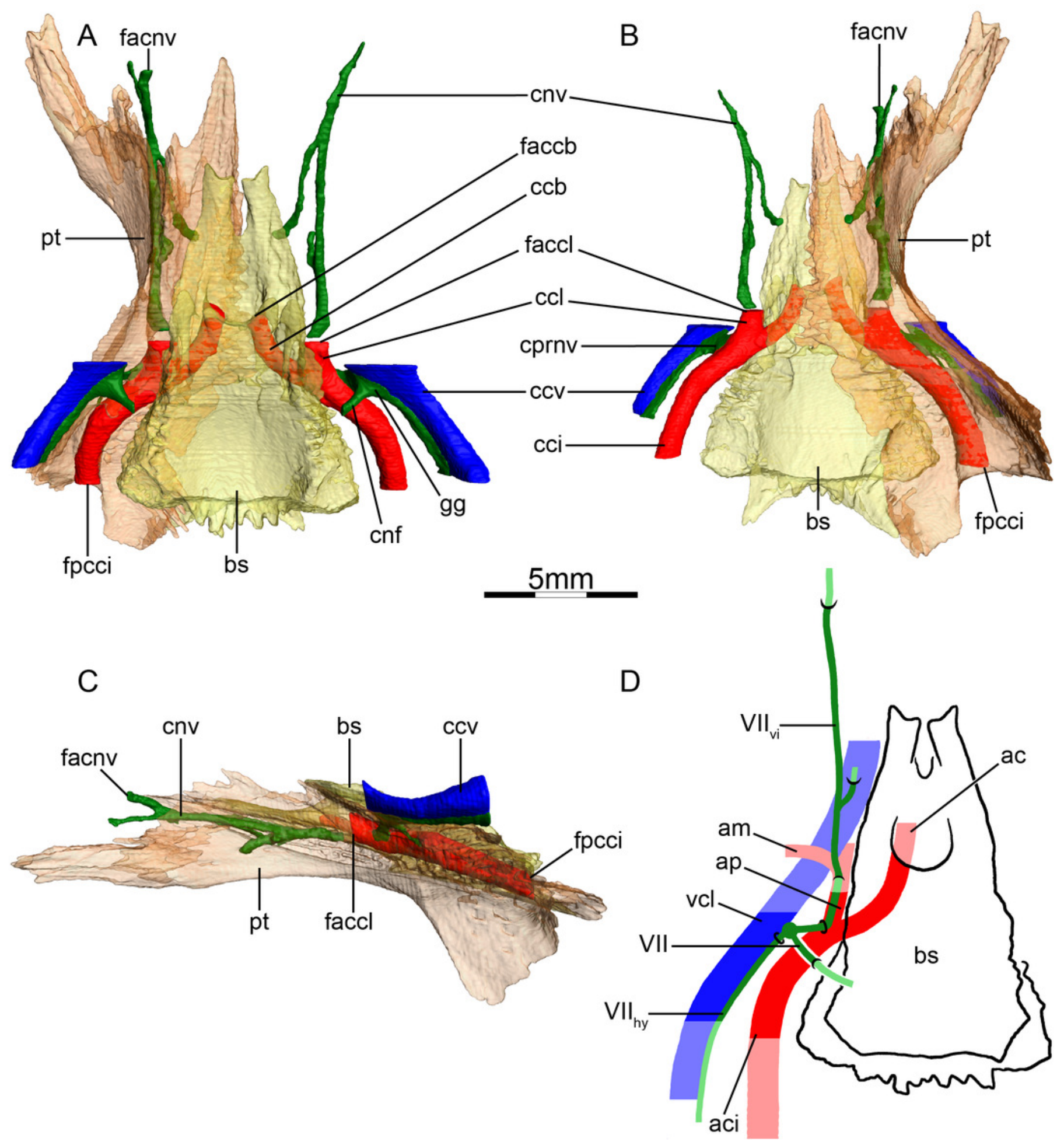




\section{Figure 9}

The carotid circulation and vidian canal system of Dermatemys mawii (SMF 59463).

Three-dimensional reconstructions of the basisphenoid, right pterygoid, in (A) dorsal, (B) ventral, and (C) left lateral view. Illustration in dorsal view (D) highlighting the placement of relevant arteries, nerves, and veins. Dark colors highlight sections fully covered by bone, light colors partially or fully uncovered sections. The red portion of the canalis cavernosus shows the inferred position of the mandibular artery. Abbreviations: ac, arteria carotis cerebralis; aci, arteria carotis interna; am-a, anterior arteria mandibularis; am-p, posterior arteria mandibularis; ap, arteria palatina; bs, basisphenoid; ccb, canalis caroticus basisphenoidalis; cci, canalis caroticus internus; ccl, canalis caroticus lateralis; ccv, canalis cavernosus; cnf, canalis nervus facialis; cnv, canalis nervus vidianus; faccb, foramen anterius canalis carotici basisphenoidalis; faccl, foramen anterius canalis caroticus lateralis; facnv, foramen anterius canalis nervi vidiani; fpcci, foramen posterius canalis carotici interni; gg, geniculate ganglion; pt, pterygoid; vcl, vena capitis lateralis; VII, nervus facialis; $\mathbf{V I I}_{\mathbf{h y}}$, nervus hyomandibularis; $\mathbf{V I I}_{\mathbf{v i}}$, nervus vidiani. 


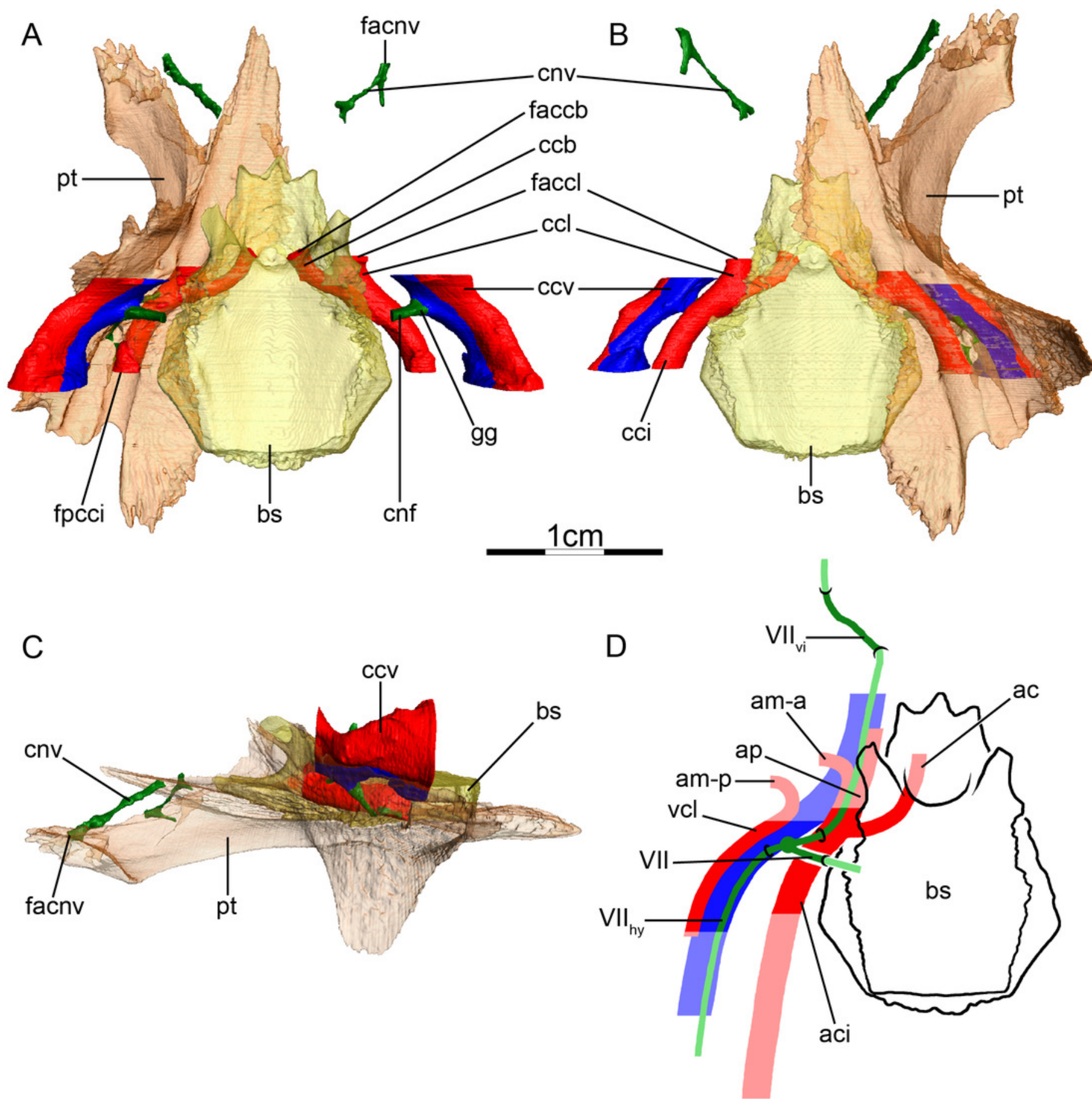




\section{Figure 10}

The carotid circulation and vidian canal system of Chelydra serpentina (SMF 32846).

Three-dimensional reconstructions of the basisphenoid, right pterygoid, in (A) dorsal, (B) ventral, and (C) left lateral view. Illustration in dorsal view (D) highlighting the placement of relevant arteries, nerves, and veins. Dark colors highlight sections fully covered by bone, light colors partially or fully uncovered sections. The red portion of the canalis cavernosus shows the inferred position of the mandibular artery. Abbreviations: ac, arteria carotis cerebralis; aci, arteria carotis interna; ap, arteria palatina; am, arteria mandibularis; bs, basisphenoid; ccb, canalis caroticus basisphenoidalis; cci, canalis caroticus internus; ccl, canalis caroticus lateralis; ccv, canalis cavernosus; cnf, canalis nervus facialis; cnv, canalis nervus vidianus; faccb, foramen anterius canalis carotici basisphenoidalis; faccl, foramen anterius canalis caroticus lateralis; facnv, foramen anterius canalis nervi vidiani; fpcci, foramen posterius canalis carotici interni; gg, geniculate ganglion; pt, pterygoid; vcl, vena capitis lateralis; $\mathbf{V I I}$, nervus facialis; $\mathbf{V I I}_{\mathbf{h y}}$, nervus hyomandibularis; $\mathbf{V I I}_{\mathbf{v i}}$, nervus vidiani. 


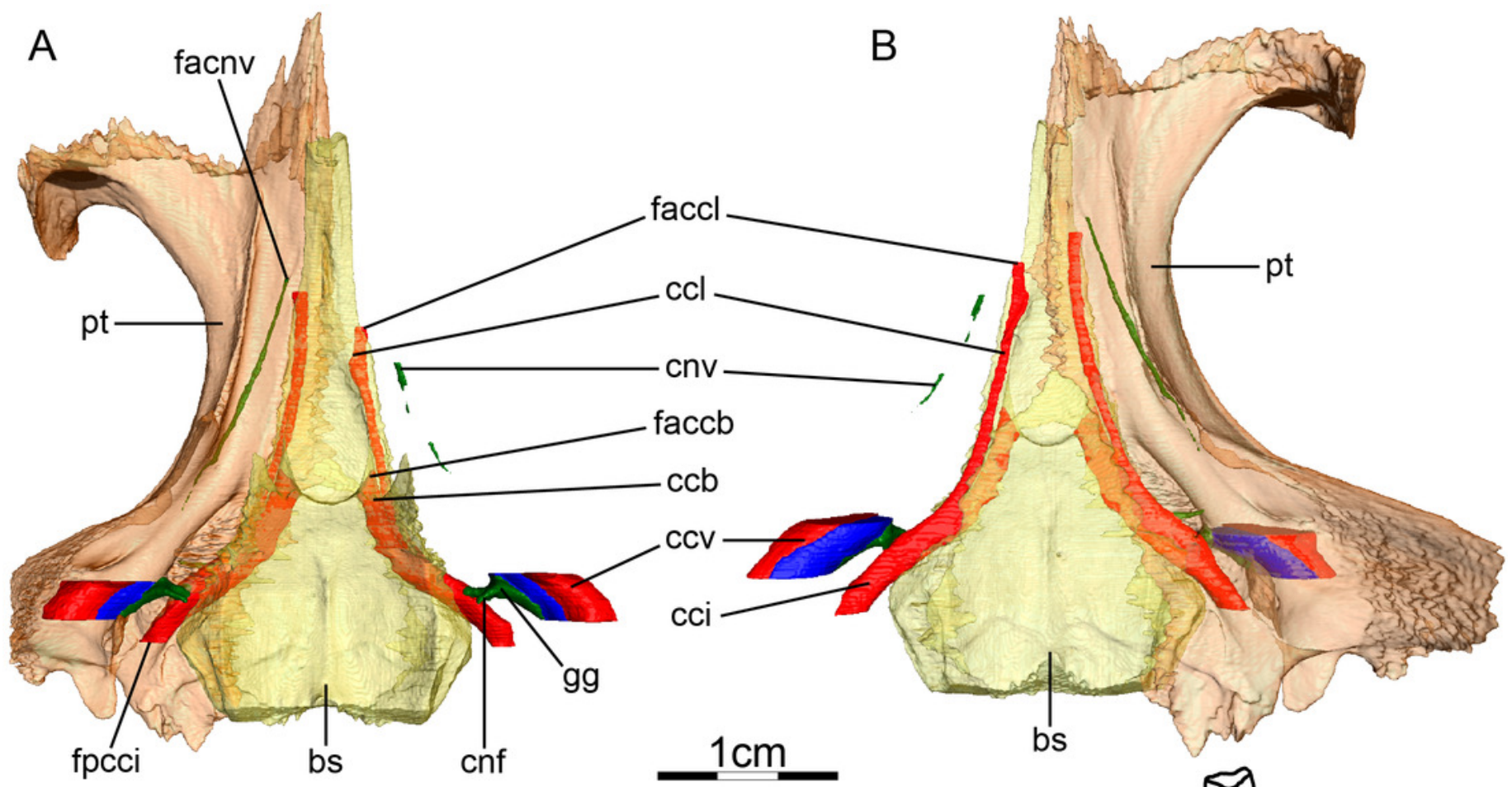

C

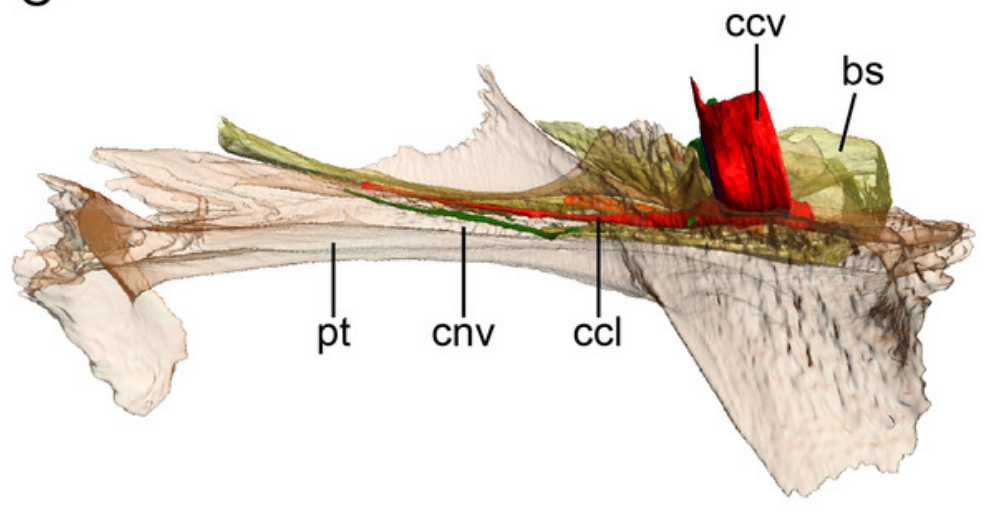

D

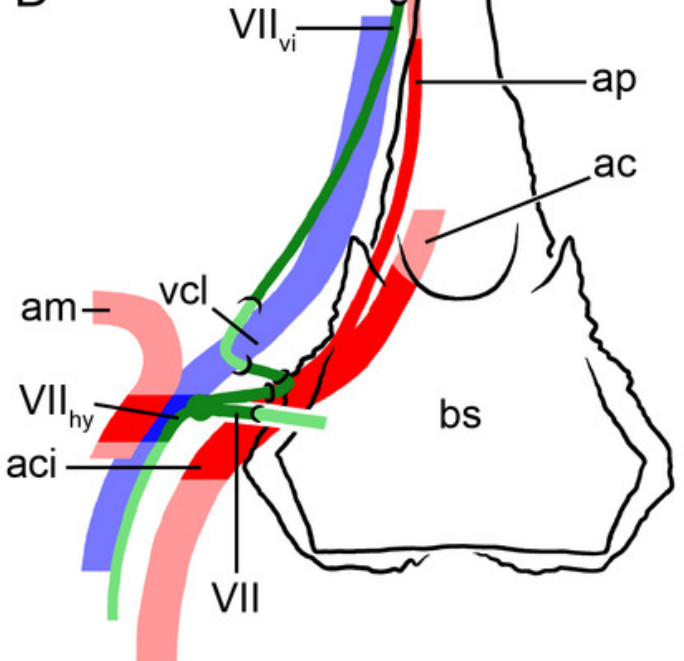




\section{Figure 11}

The carotid circulation and vidian canal system of Eretmochelys imbricata (FMNH 22242).

Three-dimensional reconstructions of the basisphenoid, right pterygoid, in (A) dorsal, (B) ventral, and (C) left lateral view. Illustration in dorsal view (D) highlighting the placement of relevant arteries, nerves, and veins. Dark colors highlight sections fully covered by bone, light colors partially or fully uncovered sections. The red portion of the canalis cavernosus shows the inferred position of the posterior mandibular artery. Abbreviations: ac, arteria carotis cerebralis; aci, arteria carotis interna; ap, arteria palatina; am-a, anterior arteria mandibularis; am-p, posterior arteria mandibularis; bs, basisphenoid; ccb, canalis caroticus basisphenoidalis; cci, canalis caroticus internus; ccv, canalis cavernosus; cnf, canalis nervus facialis; cprnv, canalis pro ramo nervi vidiani; faccb, foramen anterius canalis carotici basisphenoidalis; fpcci, foramen posterius canalis carotici interni; gg, geniculate ganglion; pt, pterygoid; v-am, vestigial arteria mandibularis; vcl, vena capitis lateralis; VII, nervus facialis; $\mathbf{V I I}_{\text {hy }}$, nervus hyomandibularis; $\mathbf{V I I}_{\mathbf{v i}}$, nervus vidiani. 


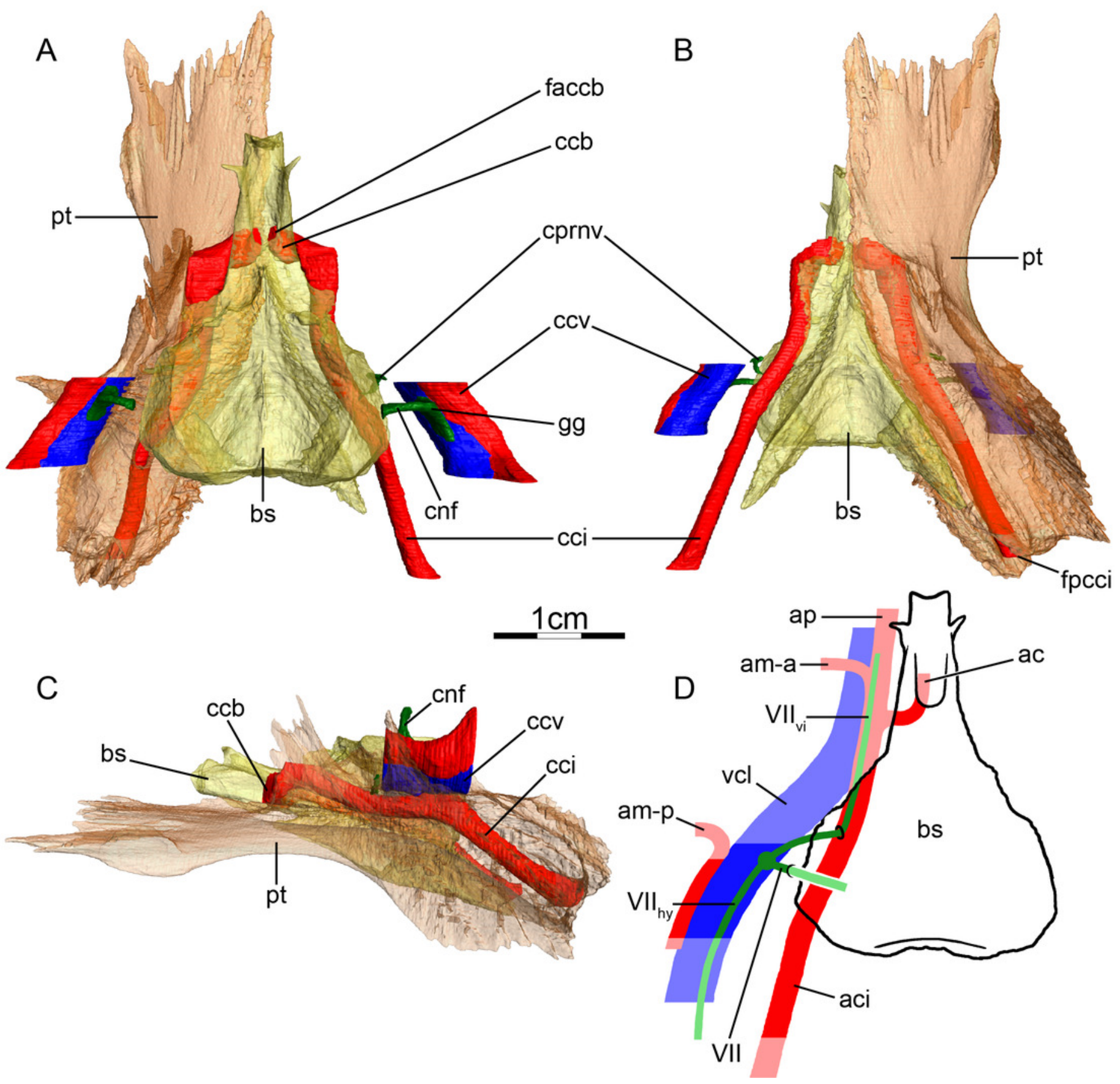




\section{Figure 12}

The carotid circulation and vidian canal system of Dermochelys coriacea (FMNH 171756).

Three-dimensional reconstructions of the basisphenoid, right pterygoid, in (A) dorsal, (B) ventral, and (C) left lateral view. Illustration in dorsal view (D) highlighting the placement of relevant arteries, nerves, and veins. Dark colors highlight sections fully covered by bone, light colors partially or fully uncovered sections. Abbreviations: ac, arteria carotis cerebralis; aci, arteria carotis interna; am, arteria mandibularis; ap, arteria palatina; bs, basisphenoid; cci, canalis caroticus internus; ccv, canalis cavernosus; cnf, canalis nervus facialis; pt, pterygoid; vcl, vena capitis lateralis; $\mathbf{V I I}$, nervus facialis; $\mathbf{V I I}_{\mathbf{h y}}$, nervus hyomandibularis; $\mathbf{V I I} \mathbf{v i}_{\mathbf{v}}$, nervus vidiani. Note that we do not highlight the course of the mandibular artery, because the mandibular blood supply of Dermochelys coriacea is currently unknown. 


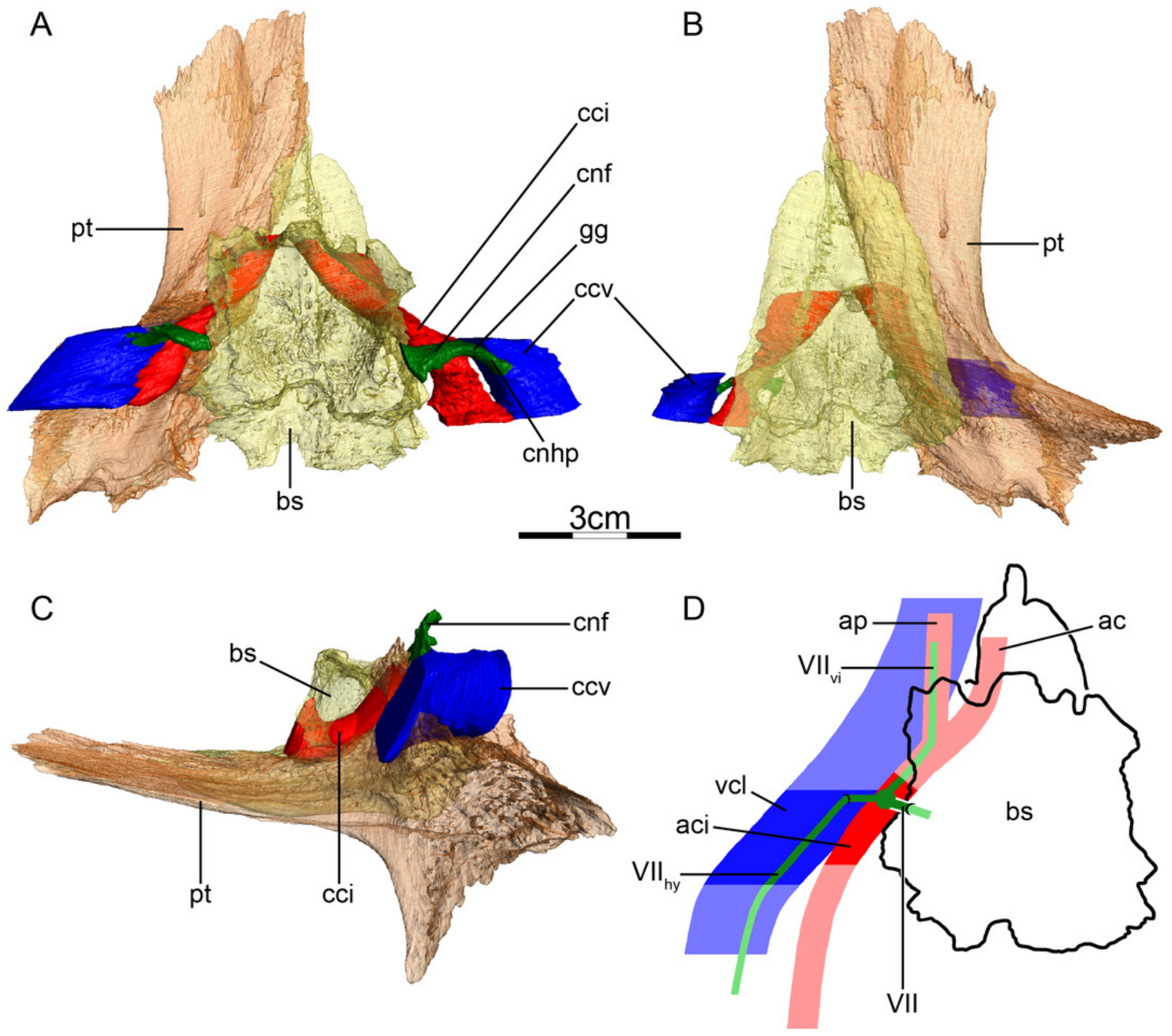




\section{Figure 13}

The carotid circulation and vidian canal system of Platysternon megacephalum (SMF 69684).

Three-dimensional reconstructions of the basisphenoid, right pterygoid, in (A) dorsal, (B) ventral, and (C) left lateral view. Illustration in dorsal view (D) highlighting the placement of relevant arteries, nerves, and veins. Dark colors highlight sections fully covered by bone, light colors partially or fully uncovered sections. The red portion of the canalis cavernosus shows the inferred position of the mandibular artery. Abbreviations: ac, arteria carotis cerebralis; aci, arteria carotis interna; am, arteria mandibularis; bs, basisphenoid; ccb, canalis caroticus basisphenoidalis; cci, canalis caroticus internus; ccv, canalis cavernosus; cnf, canalis nervus facialis; cnv, canalis nervus vidianus; cprnv, canalis pro ramo nervi vidiani; faccb, foramen anterius canalis carotici basisphenoidalis; facnv, foramen anterius canalis nervi vidiani; fpcci, foramen posterius canalis carotici interni; gg, geniculate ganglion; pt, pterygoid; vcl, vena capitis lateralis; $\mathbf{V I I}$, nervus facialis; $\mathbf{V I I}_{\mathbf{h y}}$, nervus hyomandibularis; $\mathbf{V I I}_{\mathbf{v i}}$, nervus vidiani. 


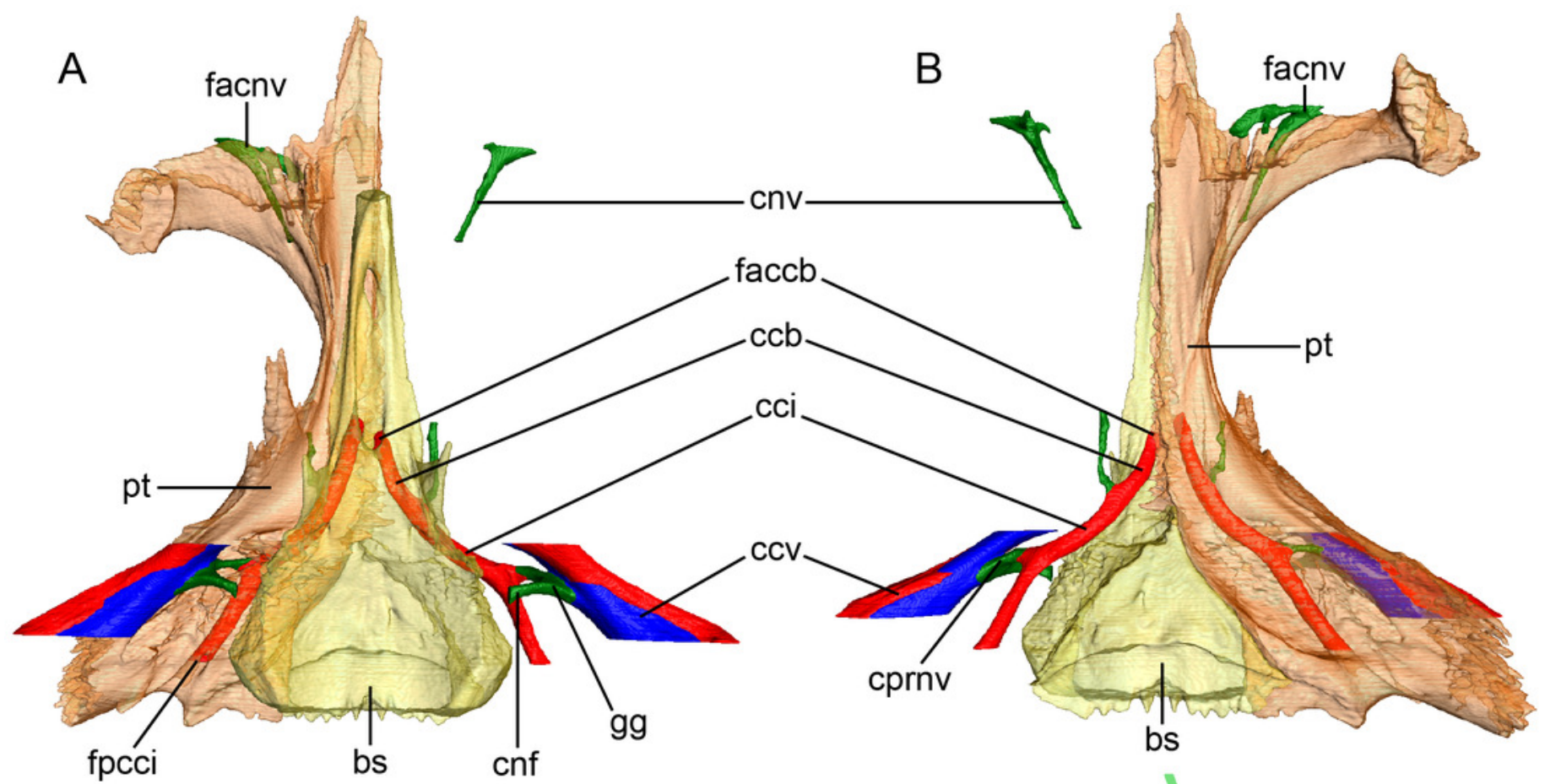

C

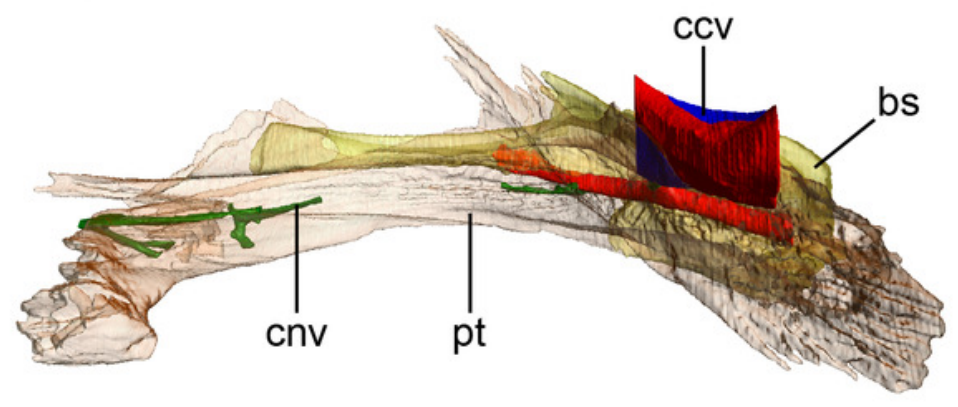

D

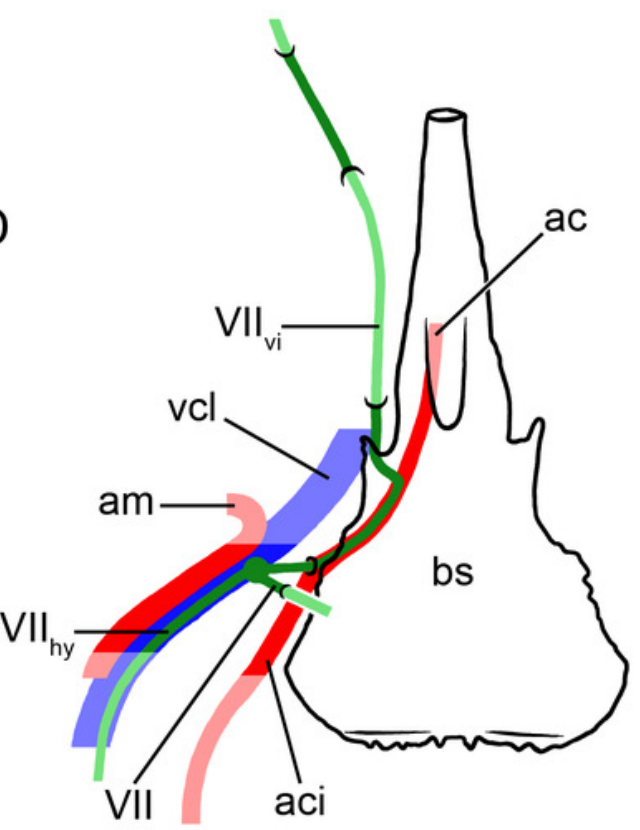




\section{Figure 14}

The carotid circulation and vidian canal system of Glyptemys insculpta (FMNH 22240).

Three-dimensional reconstructions of the basisphenoid, right pterygoid, in (A) dorsal, (B) ventral, and (C) left lateral view. Illustration in dorsal view (D) highlighting the placement of relevant arteries, nerves, and veins. Dark colors highlight sections fully covered by bone, light colors partially or fully uncovered sections. The red portion of the canalis cavernosus shows the inferred position of the mandibular artery. Abbreviations: ac, arteria carotis cerebralis; aci, arteria carotis interna; ap, arteria palatina; am, arteria mandibularis; bs, basisphenoid; ccb, canalis caroticus basisphenoidalis; cci, canalis caroticus internus; ccl, canalis caroticus lateralis; ccv, canalis cavernosus; cnf, canalis nervus facialis; cnv, canalis nervus vidianus; cprnv, canalis pro ramo nervi vidiani; faccb, foramen anterius canalis carotici basisphenoidalis; faccl, foramen anterius canalis caroticus lateralis; facnv, foramen anterius canalis nervi vidiani; fpcci, foramen posterius canalis carotici interni; gg, geniculate ganglion; pt, pterygoid; vcl, vena capitis lateralis; VII, nervus facialis; $\mathbf{V I I}_{\mathbf{h y}}$, nervus hyomandibularis; $\mathbf{V I I}_{\mathbf{v i}}$, nervus vidiani. 


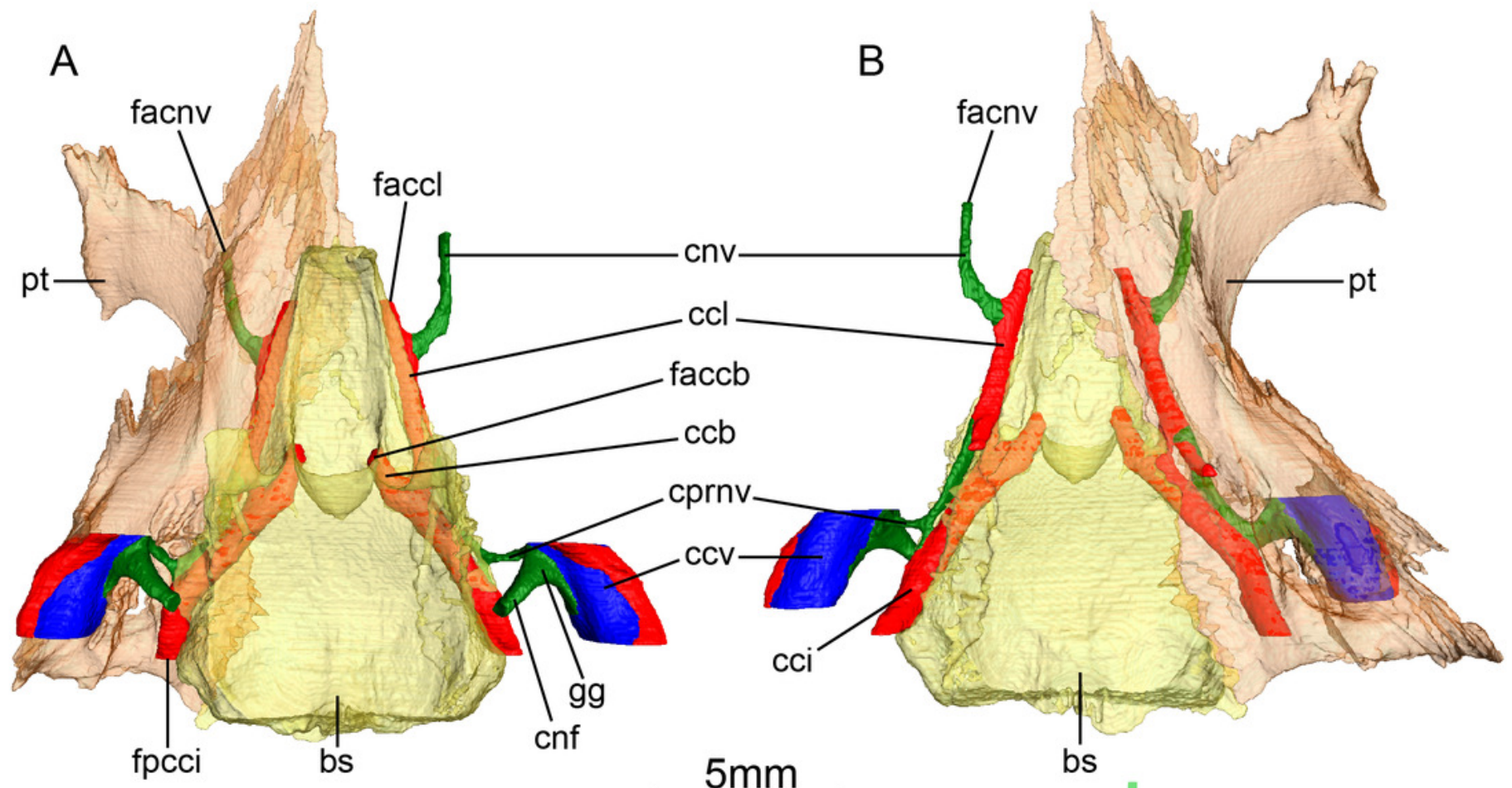

C

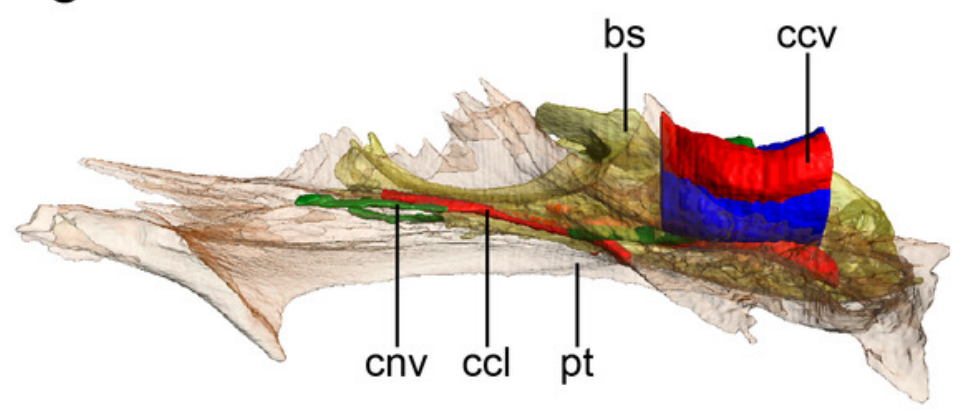

D

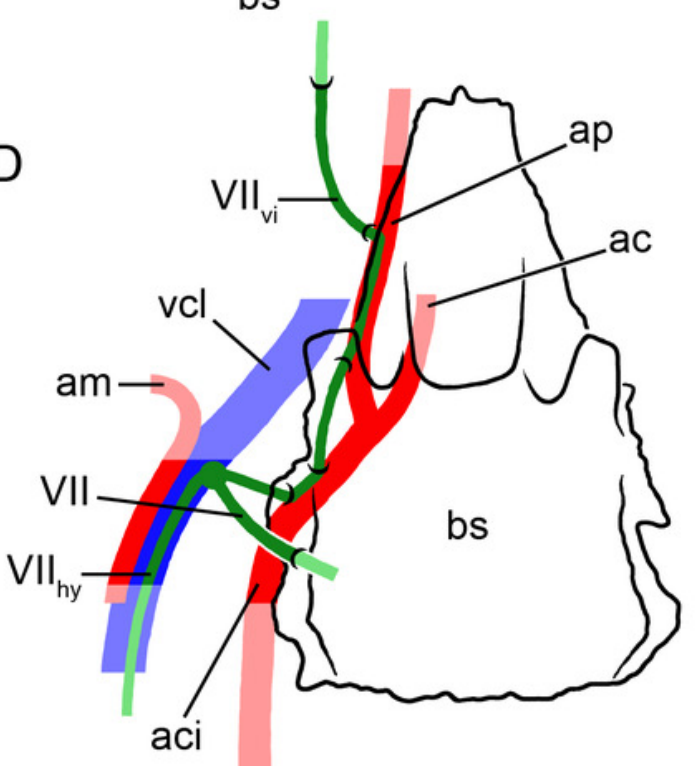




\section{Figure 15}

The carotid circulation and vidian canal system of Gopherus agassizii (FMNH 216746).

Three-dimensional reconstructions of the basisphenoid, right pterygoid, in (A) dorsal, (B) ventral, and (C) left lateral view. Illustration in dorsal view (D) highlighting the placement of relevant arteries, nerves, and veins. Dark colors highlight sections fully covered by bone, light colors partially or fully uncovered sections. The red portion of the canalis cavernosus shows the inferred position of the mandibular artery. Abbreviations: ac, arteria carotis cerebralis; aci, arteria carotis interna; am, arteria mandibularis; bs, basisphenoid; ccb, canalis caroticus basisphenoidalis; cci, canalis caroticus internus; ccv, canalis cavernosus; cnf, canalis nervus facialis; cnv, canalis nervus vidianus; faccb, foramen anterius canalis carotici basisphenoidalis; facnv, foramen anterius canalis nervi vidiani; fam, foramen arteriomandibulare; fpcci, foramen posterius canalis carotici interni; gg, geniculate ganglion; pt, pterygoid; vcl, vena capitis lateralis; $\mathbf{V I I}$, nervus facialis; $\mathbf{V I I}_{\mathbf{h y}}$, nervus hyomandibularis; $\mathbf{V I I}_{\mathbf{v i}}$, nervus vidiani. 


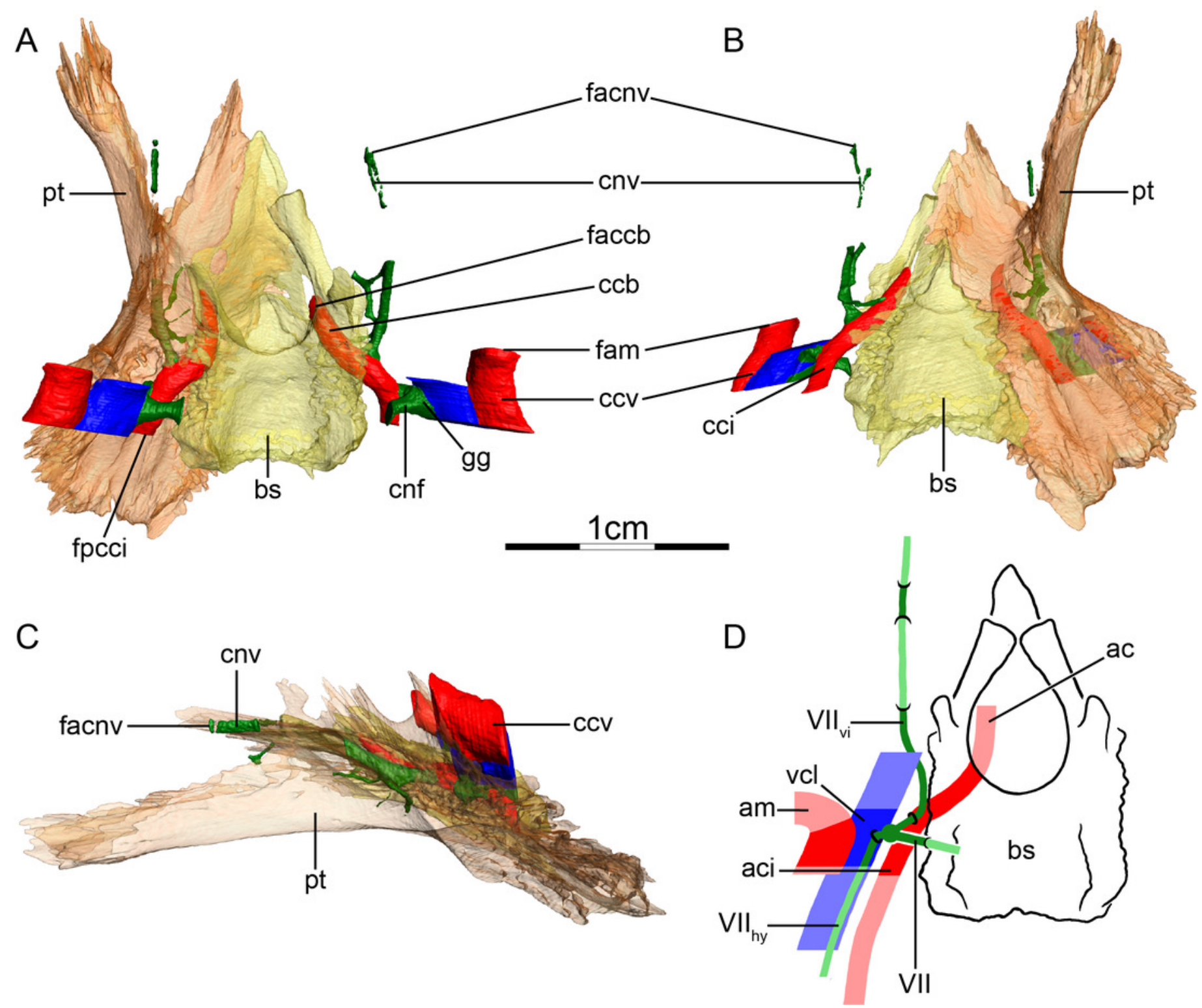




\section{Figure 16}

The carotid circulation and vidian canal system of Batagur baska (NHMUK 67.9.28.7).

Three-dimensional reconstructions of the basisphenoid, right pterygoid, in (A) dorsal, (B) ventral, and (C) left lateral view. Illustration in dorsal view (D) highlighting the placement of relevant arteries, nerves, and veins. Dark colors highlight sections fully covered by bone, light colors partially or fully uncovered sections. The red portion of the canalis cavernosus shows the inferred position of the mandibular artery. Abbreviations: ac, arteria carotis cerebralis; aci, arteria carotis interna; ap, arteria palatina; am, arteria mandibularis; bs, basisphenoid; ccb, canalis caroticus basisphenoidalis; cci, canalis caroticus internus; ccl, canalis caroticus lateralis; ccv, canalis cavernosus; cnf, canalis nervus facialis; cnv, canalis nervi vidiani; cprnv, canalis pro ramo nervi vidiani; faccb, foramen anterius canalis caroticus cerebralis; faccl, foramen anterius canalis caroticus lateralis; facnv, foramen anterius canalis nervi vidiani; fpcci, foramen posterius canalis carotici interni; gg, geniculate ganglion; pt, pterygoid; vcl, vena capitis lateralis; VII, nervus facialis; $\mathbf{V I I}_{\mathbf{h y}}$, nervus hyomandibularis; $\mathbf{V I I}_{\mathbf{v i}}$, nervus vidiani. 


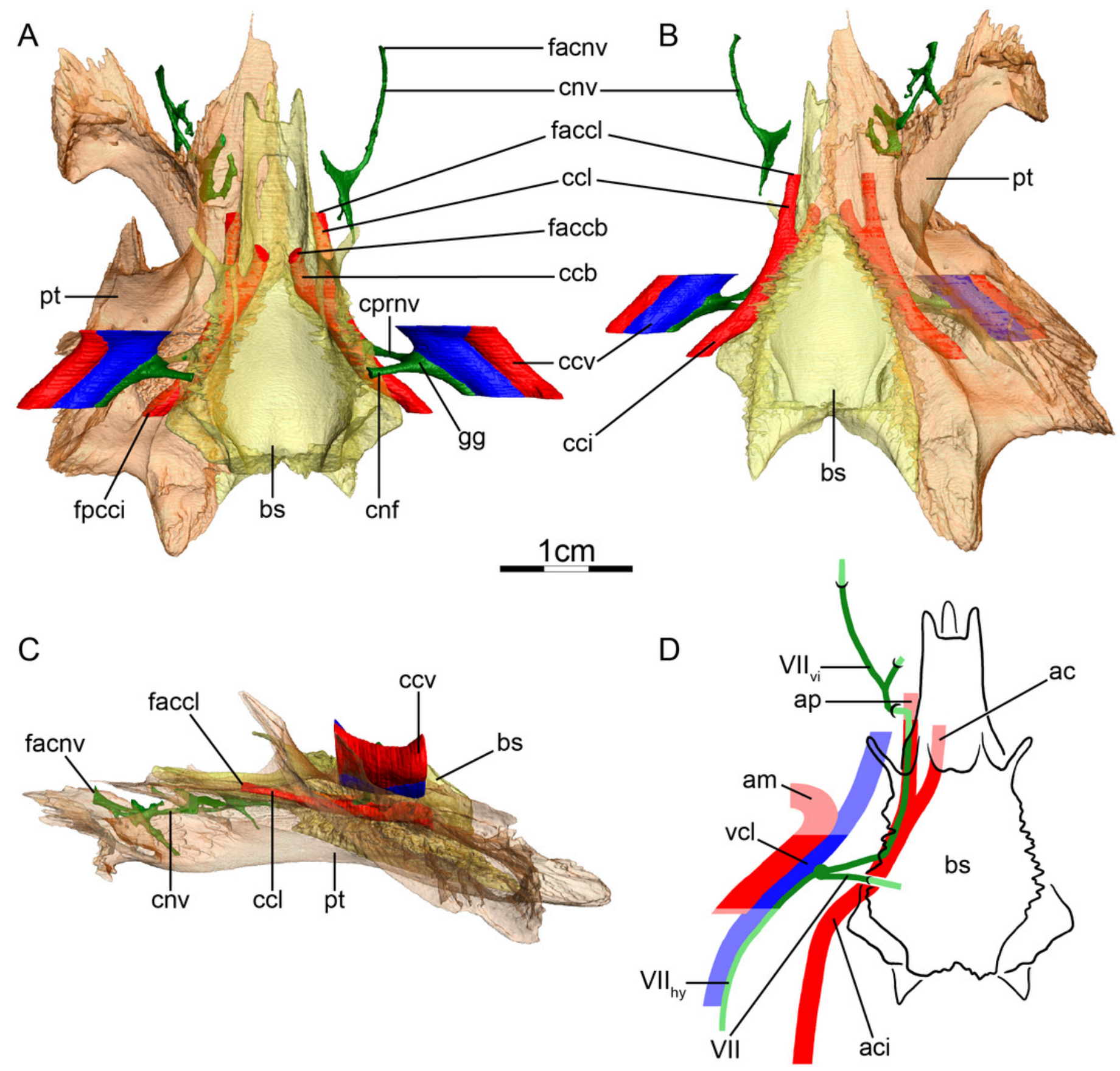




\section{Figure 17}

Asymmetry in osteological correlates for the palatine artery in Manouria impressa (SMF 69777).

(A), dorsal view of horizontally cut basicranium for orientation. (B), as A, but zoomed in on details of anterior exiting foramina for the carotid arterial system. (C), cranium in left lateral view, showing position of axial slices shown in D-E. (D), axial CT slice at position of foramina anterius canalis carotici basisphenoidalis. (E), axial CT slice at position of carotid split. Note that canals and foramina for the palatine artery are present on the right side, although the palatine artery is generally absent in testudinids. Abbreviations: bo, blind opening (opens into bone, but does not connect to blood or nervous system); ccb, canalis caroticus basisphenoidalis; faccb, foramen anterius canalis carotici basisphenoidalis; faccl, foramen anterius canalis carotici lateralis; $\mathbf{r}$-ccl, right canalis caroticus lateralis; $\mathbf{t c b}$, trabeculae of cancellous bone (small internal openings not connect to blood or nervous system). 


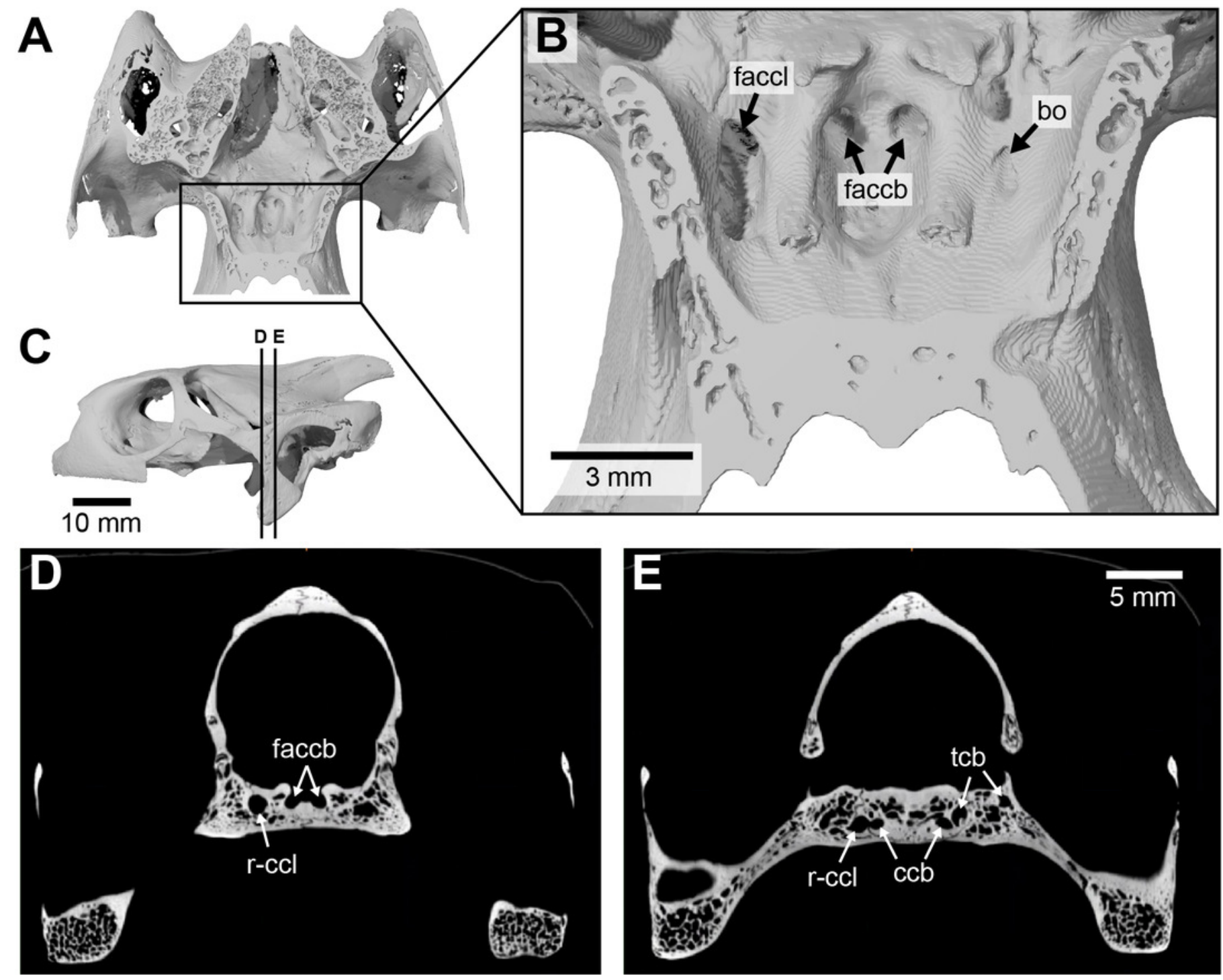




\section{Figure 18}

Schematic overview of patterns pertaining to the split of the facial nerve into its hyomandibular and vidian branches.

Abbreviations: caj, cavum acustico-jugulare; ccv, canalis cavernosus; cci, canalis caroticus internus; ccl, canalis caroticus lateralis; cnf, canalis nervus facialis; cnhp, canalis nervus hyomandibularis proximalis; cnv, canalis nervus vidianus; cprnv, canalis pro ramo nervi vidiani; faf, fossa acustico-facialis; fpcci, foramen posterius canalis carotici interni; $\mathbf{g g}$, geniculate ganglion; VII, nervus facialis; $\mathbf{V I I}_{\mathbf{h y}}$, nervus hyomandibularis; $\mathbf{V I I}_{\mathbf{v i}}$, nervus vidiani. 

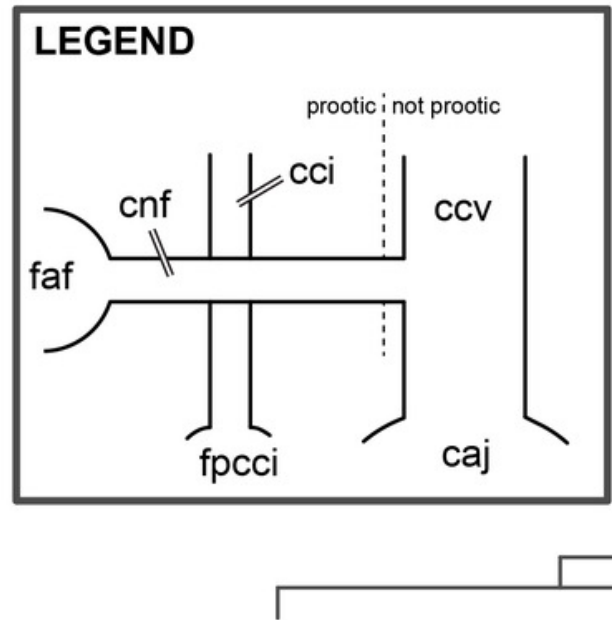

FACIAL PATTERN IA

canalis pro ramo nervi vidiani transmits vidian to int. carotid

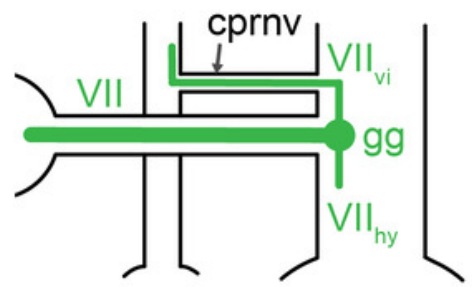

Chelydroidea, Cheloniidae, most emysternids, most geoemydids, some testudinids, some trionychids

\section{FACIAL PATTERN I}

Ganglion outside of prootic \& inside canalis cavernosus

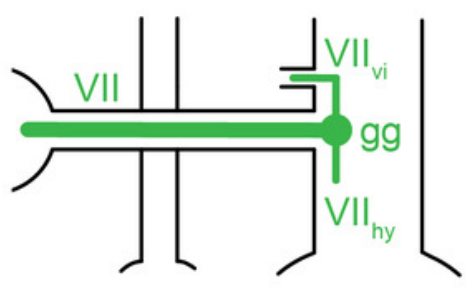

FACIAL PATTERN II

Ganglion inside prootic; lateral continuation of canalis nervus facialis for hyomandibular nerve to canalis cavernosus present

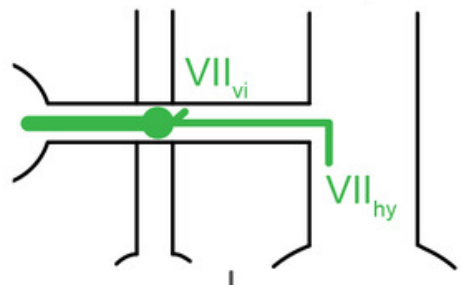

\section{FACIAL PATTERN IC} canalis pro ramo nervi vidiani transmits vidian to pal. canal

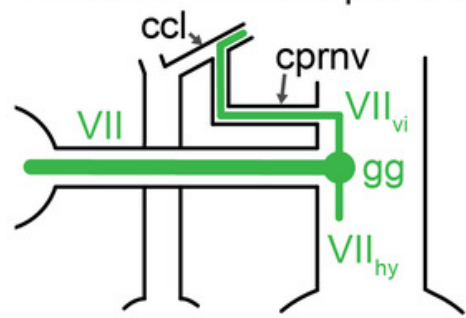

some emydids, some geoemydids canalis nervus vidianus exits directly from cavernosus

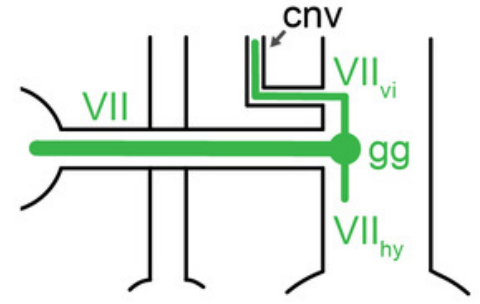

some testudinids

\section{FACIAL PATTERN IIA}

Vidian nerve transmitted from ganglion to canalis caroticus internus by separate canal

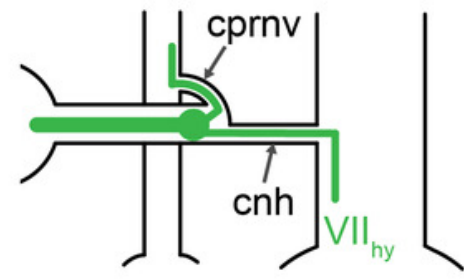

Carettochelys insculpta, some trionychids, some testudinids, podocnemidids

\section{FACIAL PATTERN IIB}

Vidian nerve enters canalis caroticus internus directly ('ganglion within cci')

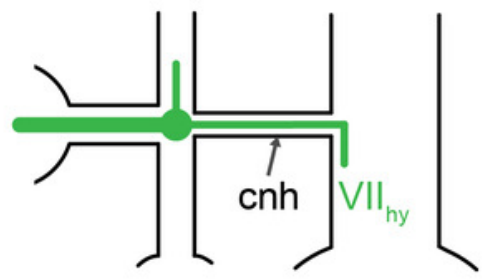

most chelids, Pelomedusa subrufa Dermochelys coriacea
FACIAL PATTERN IIC

Vidian nerve enters canalis nervus vidianus directly

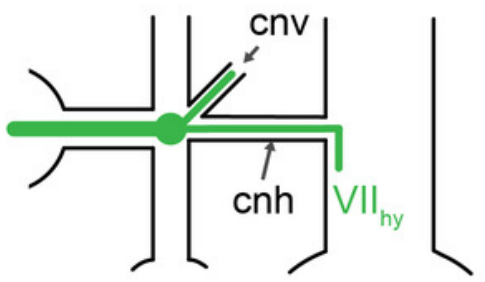

Chelus fimbriatus, Pelusios subniger 


\section{Figure 19}

Schematic overview of patterns pertaining to the posterior portion of the hyomandibular nerve.

The five patterns presented herein only apply to taxa in which the vidian nerve enters the carotid canal system (i.e. turtles with patterns IA, IIA, and IIB). Abbreviations: caj, cavum acustico-jugulare; ccv, canalis cavernosus; cnhd, canalis nervus hyomandibularis distalis; snh, sulcus nervus hyomandibularis; $\mathbf{V I I}_{\mathbf{h y}}$, nervus hyomandibularis.

HYOMANDIBULAR PATTERN I

Distal part of hyomandibular nerve in separate canal paralleling canalis cavernosus

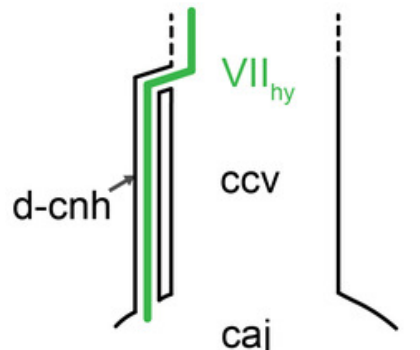

HYOMANDIBULAR

PATTERN II

Distal part of hyomandibular nerve in sulcus in wall of canalis cavernosus

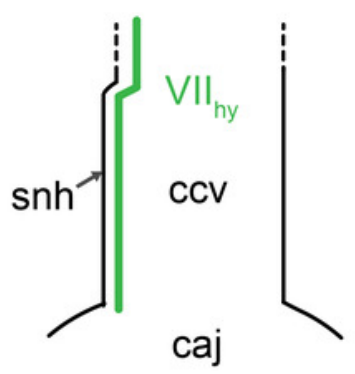

HYOMANDIBULAR

PATTERN III

Distal part of hyomandibular nerve in canalis cavernosus; no osteological correlate

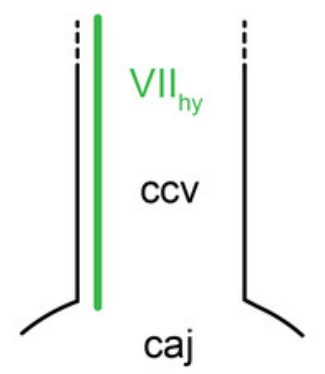


Figure 20

Schematic overview of patterns pertaining to the anterior portion of the vidian nerve.

Abbreviations: $\mathbf{c c i}$, canalis caroticus internus; $\mathbf{c c l}$, canalis caroticus lateralis; $\mathbf{c n v}$, canalis nervus vidianus; fpcci, foramen posterius canalis carotici interni; scc, "short cut canal"; scv, sulcus cavernosus; $\mathbf{V I I}$ vi , vidian nerve.

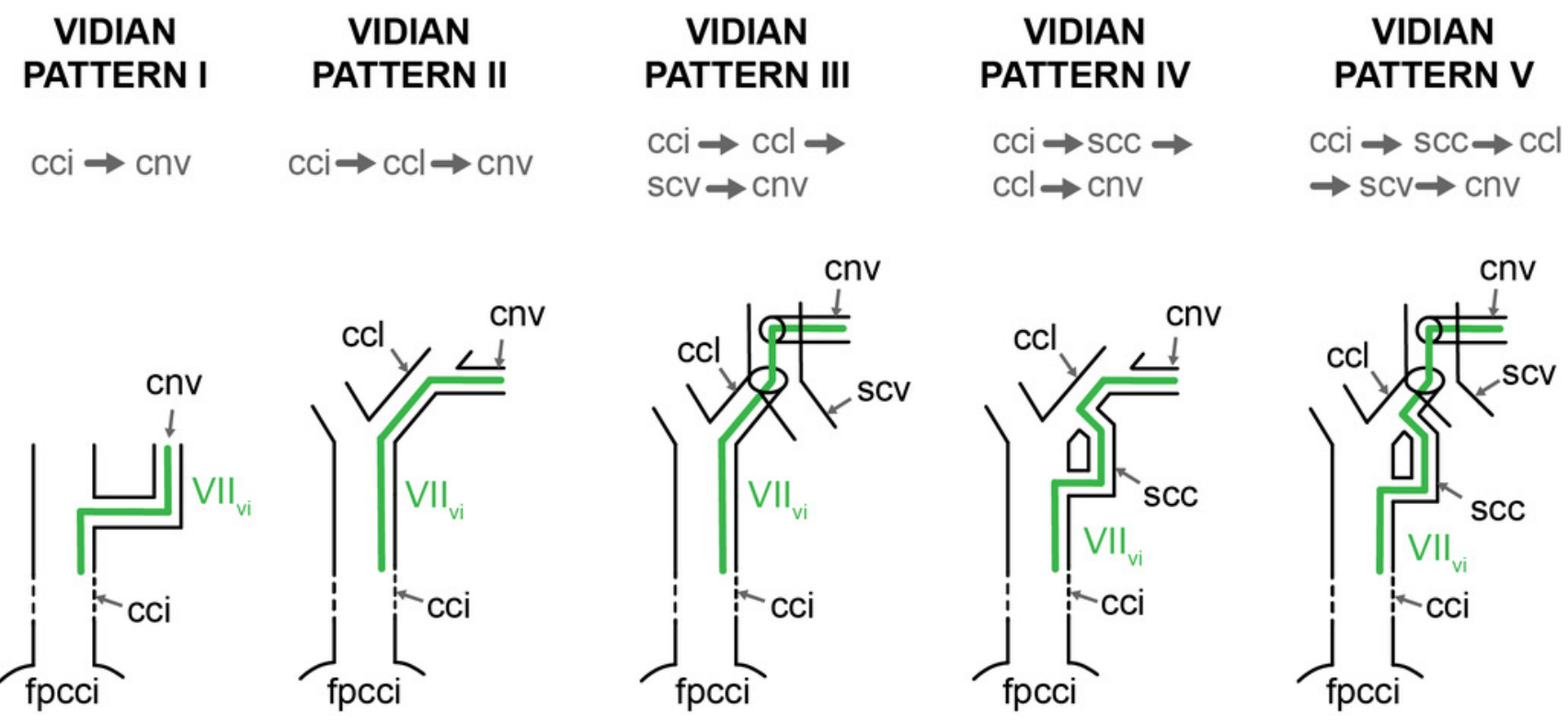


Figure 21

Relationship of internal carotid artery size and stapedial artery size in turtles.

(A), pGLS regression of $\log _{10^{-}}$Cross-sectional diameter of internal carotid artery canal on $\log _{10^{-}}$ cross-sectional diameter of stapedial artery canal. Solid grey line is the regression line describing a model with a single slope and intercept for all taxa. Dashed grey lines are regression lines for subsets of the data (see text and table 3 for details). (B), plot showing which data points were used as subsets for the multiple regression model test. Green points represent the subset containing kinosternoids, chelonioids, and trionychians, whereas blue points represent the subset containing the remaining turtle clades. (C), residual plot of pGLS regression of the full dataset, ordered by clades. Symbols in A and C denote clade attributions.

A

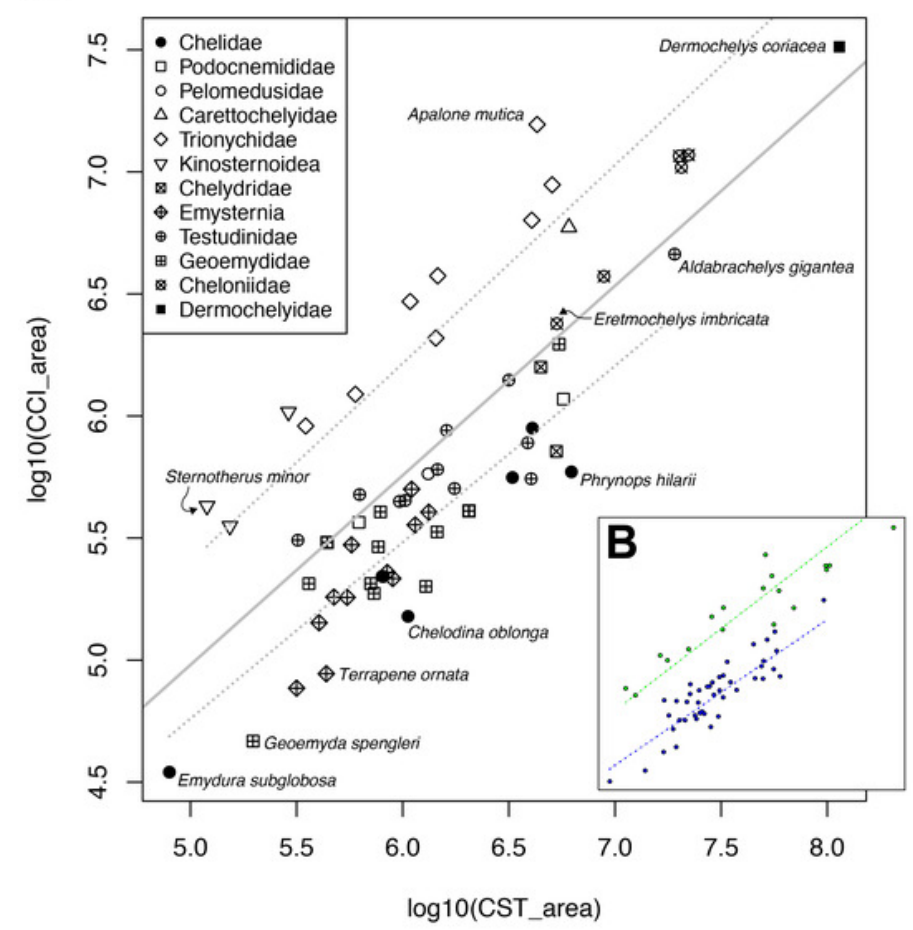

C

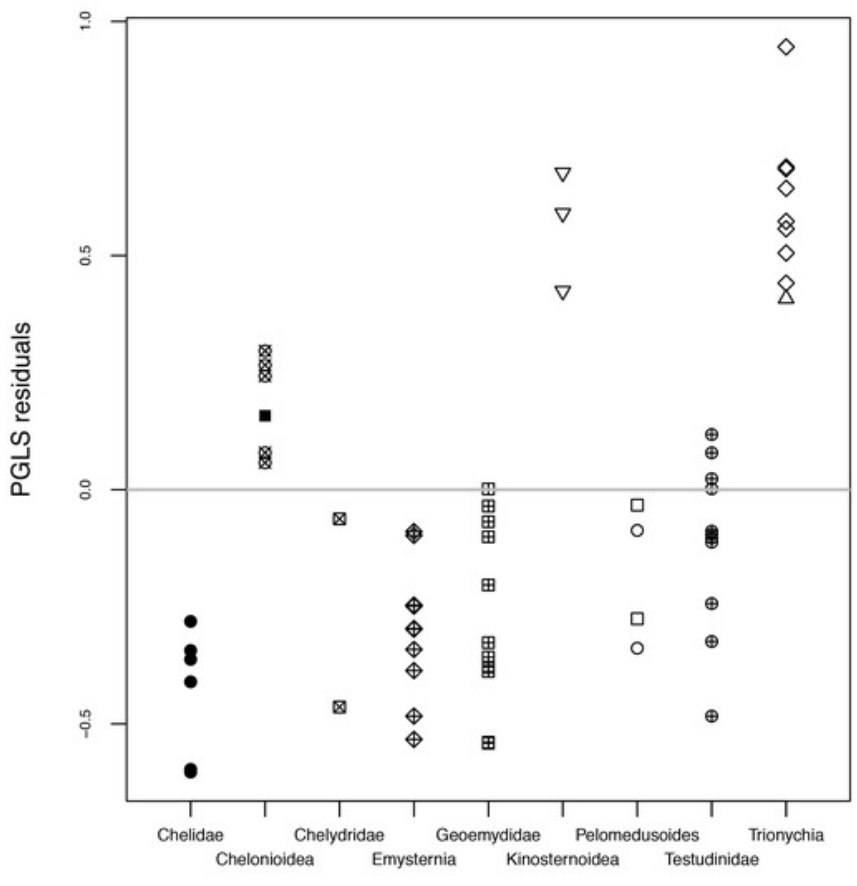




\section{Table $\mathbf{1}$ (on next page)}

List of specimens used in this study.

Abbreviations: FMNH, Field Museum of Natural History, Chicago, IL, USA; NHMUK, Natural History Museum London, London, England; NMB, Naturhistorisches Museum Basel, Basel, Switzerland; PCHP, Chelonian Research Institute, Oviedo, FL, USA; PIMUZ, Paläontologisches Museum Zürich, Zürich, Switzerland; SMF, Senckenberg Naturmuseum Frankfurt, Frankfurt am Main, Germany; SMNS, Staatliches Museum für Naturkunde Stuttgart, Stuttgart, Germany; UF, Florida Museum of Natural History, Gainesville, FL, USA; UMZC, University Museum of Zoology Cambridge, Cambridge, England. 


\begin{tabular}{|c|c|c|c|c|c|}
\hline Taxon & Specimen(s) used & Repository & Project & $\begin{array}{l}\text { Media accession } \\
\text { number }\end{array}$ & Citation/Source \\
\hline Carettochelys insculpta & NHMUK 1903.7.10.1 & MorphoSource & P769 & M42840 & Evers (2019) \\
\hline \multicolumn{6}{|l|}{ Chelidae } \\
\hline Chelodina oblonga & NHMUK 64.12 .22 & MorphoSource & P769 & M42849 & Evers (2019) \\
\hline Chelus fimbriatus & NHMUK 81.9.27.4 & MorphoSource & P769 & M42856 & Evers (2019) \\
\hline Phrynops geoffroanus & SMF 45470 & MorphoSource & P462 & M22118 & Evers \& Benson (2018) \\
\hline Phrynops hilarii & NHMUK 91.3.16.1 & MorphoSource & P769 & M42860 & Evers (2019) \\
\hline \multicolumn{6}{|l|}{ Cheloniidae } \\
\hline Caretta caretta & NHMUK 1938.1.9.1 & MorphoSource & P769 & M42843 & Evers (2019) \\
\hline Caretta caretta & NHMUK 1940.3.15.1 & MorphoSource & P769 & M42844 & Evers (2019) \\
\hline \multicolumn{6}{|l|}{ Chelydridae } \\
\hline Chelydra serpentina & SMF 32846 & none & NA & NA & shared by I. Werneburg \\
\hline Macrochelys temminckii & FMNH 22111 & MorphoSource & P769 & M42869 & Evers (2019) \\
\hline \multicolumn{6}{|l|}{ Dermatemydidae } \\
\hline Dermatemys mawii & SMF 59463 & none & NA & NA & shared by I. Werneburg \\
\hline \multicolumn{6}{|l|}{ Dermochelyidae } \\
\hline Dermochelys coriacea & FMNH 171756 & MorphoSource & P769 & M42864 & Evers (2019) \\
\hline Dermochelys coriacea & UMZC R3031 & MorphoSource & P462 & M22024 & Evers \& Benson (2018) \\
\hline \multicolumn{6}{|l|}{ Emydidae } \\
\hline Clemmys guttata & FMNH 22114 & MorphoSource & P769 & M42870 & Evers (2019) \\
\hline Deirochelys reticularia & FMNH 98754 & MorphoSource & P769 & M42883 & Evers (2019) \\
\hline
\end{tabular}


Glyptemys insculpta

Glyptemys muhlenbergii

Graptemys geographica

Pseudemys floridana

Terrapene coahuila

Terrapene ornata

\section{Geoemydidae}

Batagur baska

Cuora amboinensis

Cyclemys dentata

Geoclemys hamiltonii

Geoemyda spengleri

Malayemys subtrijuga

Mauremys leprosa

Morenia ocellata

Pangshura tecta

Rhinoclemmys melanosterna

Siebenrockiella crassicollis

\section{Kinosternidae}

Kinosternon baurii

Kinosternon scorpioides

Kinosternon subrubrum hippocrepis

Staurotypus salvinii

Sternotherus minor

\section{Pelomedusidae}

Pelomedusa subrufa

Pelusios subniger

\section{Platysternidae}

Platysternon megacephalum

\section{Podocnemididae}

Podocnemis unifilis

Podocnemis unifilis

\begin{tabular}{|c|c|c|c|}
\hline FMNH 22240 & MorphoSource & P769 & M42873 \\
\hline UF 85274 & Digimorph & NA & NA \\
\hline NHMUK 55.12.6.11 & MorphoSource & P769 & M42847 \\
\hline FMNH 8222 & MorphoSource & P769 & M42882 \\
\hline FMNH 47372 & MorphoSource & P769 & M42881 \\
\hline FMNH 23014 & MorphoSource & P769 & M42875 \\
\hline NHMUK 67.9.28.7 & MorphoSource & P769 & M42851 \\
\hline NHMUK 69.42 .145 & MorphoSource & P769 & M42852 \\
\hline NHMUK 97.11.22.3 & MorphoSource & P769 & M42861 \\
\hline NHMUK 87.9.30.1 & MorphoSource & P769 & M42859 \\
\hline FMNH 260381 & MorphoSource & P769 & M42877 \\
\hline NHMUK 1920.1.20.2545 & MorphoSource & P769 & M42841 \\
\hline NHMUK unnumbered & MorphoSource & P769 & M42862 \\
\hline NHMUK 87.3.11.7 & MorphoSource & P769 & M42858 \\
\hline NHMUK 1889.2.6.1 & MorphoSource & P769 & M42839 \\
\hline FMNH 44446 & MorphoSource & P769 & M42879 \\
\hline NHMUK 1864.9.2.47 & MorphoSource & P769 & M42837 \\
\hline FMNH 211705 & MorphoSource & P769 & M42865 \\
\hline SMF 71893 & none & NA & NA \\
\hline FMNH 211711 & MorphoSource & P769 & M42866 \\
\hline NHMUK 1879.1.7.5 & MorphoSource & P769 & M42838 \\
\hline FMNH 211696 & MorphoSource & P462 & M22123 \\
\hline NMB 16229 & none & NA & NA \\
\hline NMB 16230 & none & NA & NA \\
\hline SMF 69684 & none & NA & NA \\
\hline NHMUK 60.4.16.9 & MorphoSource & P769 & M42848 \\
\hline FMNH 45657 & MorphoSource & P769 & M42880 \\
\hline
\end{tabular}

Evers (2019)

shared by J. Olori

Evers (2019)

Evers (2019)

Evers (2019)

Evers (2019)

Evers (2019)

Evers (2019)

Evers (2019)

Evers (2019)

Evers (2019)

Evers (2019)

Evers (2019)

Evers (2019)

Evers (2019)

Evers (2019)

Evers (2019)

Evers (2019)

shared by I. Werneburg

Evers (2019)

Evers (2019)

Evers \& Benson (2018)

scanned at University of Fribourg scanned at University of Fribourg

shared by I. Werneburg

Evers (2019)

Evers (2019) 


\section{Testudinidae}

Agrionemys horsfieldii

Aldabrachelys gigantea

Gopherus agassizii

Gopherus flavomarginatus

Gopherus polyphemus

Indotestudo elongata

Indotestudo forstenii

Kinixys erosa

Malacochersus tornieri

Psammobates tentorius verroxii

Testudo marginata

\section{Trionychidae}

Amyda cartilaginea

Apalone mutica

Apalone spinifera emoryi

Chitra indica

Cyclanorbis senegalensis

Cycloderma frenatum

Lissemys punctata

Pelodiscus sinensis

PCHP 2929
NHMUK 77.11.12.2
FMNH 216746
FMNH 98916
FMNH 211815
SMF 71585
SMF 73257
SMF 40166
SMF 58702
SMF 57142
FMNH 51672

$\begin{array}{lll}\text { Digimorph } & \text { NA } & \text { NA } \\ \text { MorphoSource } & \text { P769 } & \text { M42855 } \\ \text { MorphoSource } & \text { P769 } & \text { M42868 } \\ \text { MorphoSource } & \text { P769 } & \text { M42884 } \\ \text { MorphoSource } & \text { P462 } & \text { M22041 } \\ \text { none } & \text { NA } & \text { NA } \\ \text { none } & \text { NA } & \text { NA } \\ \text { none } & \text { NA } & \text { NA } \\ \text { none } & \text { NA } & \text { NA } \\ \text { none } & \text { NA } & \text { NA } \\ \text { MorphoSource } & \text { P769 } & \text { M42885 } \\ & & \\ \text { MorphoSource } & \text { P769 } & \text { M42876 } \\ \text { Digimorph } & \text { NA } & \text { NA } \\ \text { MorphoSource } & \text { P462 } & \text { M22038 } \\ \text { MorphoSource } & \text { P769 } & \text { M42842 } \\ \text { MorphoSource } & \text { P769 } & \text { M42850 } \\ \text { MorphoSource } & \text { P769 } & \text { M42857 } \\ \text { none } & \text { NA } & \text { NA } \\ \text { none } & \text { NA } & \text { NA }\end{array}$

shared by H. Jamniczky

Evers (2019)

Evers (2019)

Evers (2019)

Evers \& Benson (2018)

shared by I. Werneburg

shared by I. Werneburg

shared by I. Werneburg

Lautenschlager et al. (2018)

shared by I. Werneburg

Evers (2019)

FMNH 244117
PCHP 2746
FMNH 22178
NHMUK 1926.12.16.1
NHMUK 65.5.9.21
NHMUK 84.2.4.1
SMF 74141
IW576-2b

none

NA

NA

Evers (2019)

shared by H. Jamniczky

Evers \& Benson (2018)

Evers (2019)

Evers (2019)

Evers (2019)

shared by I. Werneburg

Lautenschlager et al. (2018) 


\section{Table 2 (on next page)}

Cross-sectional area values $\left(\mu \mathrm{m}^{2}\right)$ of the stapedial and carotid artery canals.

Abbreviations: CCB, canalis caroticus basisphenoidalis; CCl, canalis caroticus internus; CCL, canalis caroticus lateralis; CST, canalis stapedio-temporalis. Note that value 0 is used when the canalis caroticus lateralis and arteria palatina are known to be absent, whereas NA (nonapplicable) is only used for chelonioids, in which the palatine artery is present but does not extend through its own canal, preventing us from making any measurement of the latter. 


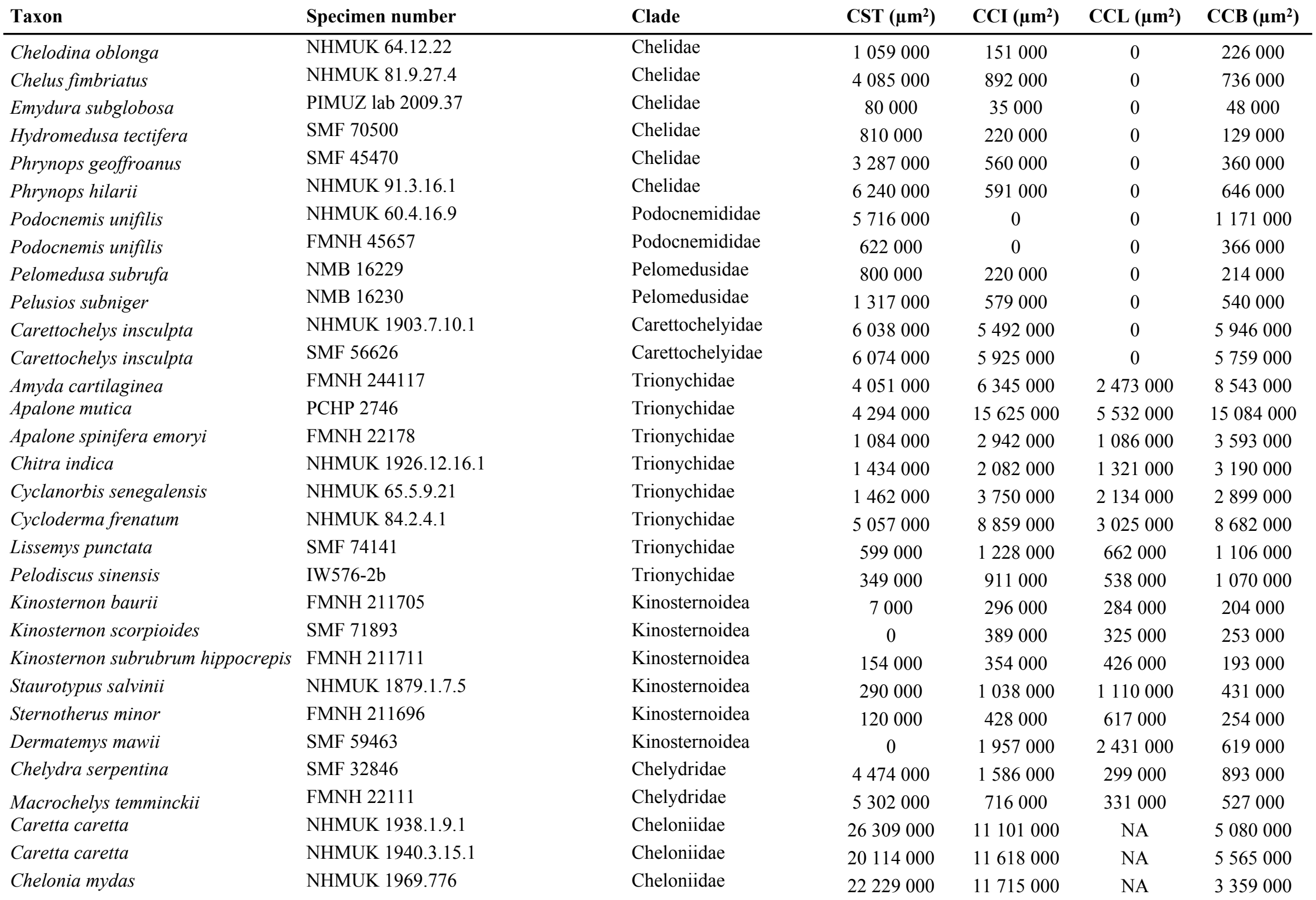




$\begin{array}{lll}\text { Eretmochelys imbricata } & \text { FMNH } 22242 & \text { Cheloniidae } \\ \text { Lepidochelys olivacea } & \text { SMNS } 11070 & \text { Cheloniidae } \\ \text { Natator depressus } & \text { R112123 } & \text { Cheloniidae } \\ \text { Dermochelys coriacea } & \text { FMNH } 171756 & \text { Dermochelyidae } \\ \text { Dermochelys coriacea } & \text { UMZC R3031 } & \text { Dermochelyidae } \\ \text { Platysternon megacephalum } & \text { SMF } 69684 & \text { Emysternia } \\ \text { Clemmys guttata } & \text { FMNH } 22114 & \text { Emysternia } \\ \text { Deirochelys reticularia } & \text { FMNH } 98754 & \text { Emysternia } \\ \text { Emydoidea blandingii } & \text { FMNH } 22144 & \text { Emysternia } \\ \text { Emys orbicularis } & \text { SMF } 1987 & \text { Emysternia } \\ \text { Glyptemys insculpta } & \text { FMNH } 22240 & \text { Emysternia } \\ \text { Glyptemys muhlenbergii } & \text { UF } 85274 & \text { Emysternia } \\ \text { Graptemys geographica } & \text { NHMUK } 55.12 .6 .11 & \text { Emysternia } \\ \text { Pseudemys floridana } & \text { FMNH } 8222 & \text { Emysternia } \\ \text { Terrapene coahuila } & \text { FMNH } 47372 & \text { Emysternia } \\ \text { Terrapene ornata } & \text { FMNH } 23014 & \text { Emysternia } \\ \text { Agrionemys horsfieldii } & \text { PCHP } 2929 & \text { Testudinidae } \\ \text { Aldabrachelys gigantea } & \text { NHMUK } 77.11 .12 .2 & \text { Testudinidae } \\ \text { Gopherus agassizii } & \text { FMNH } 216746 & \text { Testudinidae } \\ \text { Gopherus flavomarginatus } & \text { FMNH } 98916 & \text { Testudinidae } \\ \text { Gopherus polyphemus } & \text { FMNH } 211815 & \text { Testudinidae } \\ \text { Indotestudo elongata } & \text { SMF } 71585 & \text { Testudinidae } \\ \text { Indotestudo forstenii } & \text { SMF } 73257 & \text { Testudinidae } \\ \text { Kinixys erosa } & \text { SMF } 40166 & \text { Testudinidae } \\ \text { Malacochersus tornieri } & \text { SMF } 58702 & \text { Testudinidae } \\ \text { Psammobates tentorius verroxii } & \text { SMF } 57142 & \text { Testudinidae } \\ \text { Testudo marginata } & \text { FMNH } 51672 & \text { Testudinidae } \\ \text { Batagur baska } & \text { NHMUK } 67.9 .28 .7 & \text { Geoemydidae } \\ \text { Cuora amboinensis } & \text { NHMUK } 69.42 .145 & \text { Geoemydidae } \\ \text { Cyclemys dentata } & \text { NHMUK } 97.11 .22 .3 & \text { Geoemydidae } \\ \text { Geoclemys hamiltonii } & \text { NHMUK } 87.9 .30 .1 & \text { Geoemydidae } \\ \text { Geoemyda spengleri } & \text { FMNH } 260381 & \text { Geoemydidae }\end{array}$

$\begin{array}{cccc}5324000 & 2387000 & \text { NA } & 1457000 \\ 8862000 & 3737000 & \text { NA } & 1944000 \\ 20562000 & 10457000 & \text { NA } & 3624000 \\ 114072000 & 32496000 & \text { NA } & \text { NA } \\ 94064000 & 32013000 & \text { NA } & \text { NA } \\ 1327000 & 404000 & 0 & 424000 \\ 316000 & 77000 & 21000 & 69000 \\ 573000 & 296000 & 25000 & 240000 \\ 845000 & 228000 & 109000 & 167000 \\ 547000 & 181000 & 51000 & 176000 \\ 1145000 & 358000 & 109000 & 197000 \\ 1102000 & 501000 & 100000 & 590000 \\ 404000 & 142000 & 60000 & 74000 \\ 898000 & 216000 & 35000 & 171000 \\ 474000 & 181000 & 48000 & 165000 \\ 437000 & 88000 & 14000 & 74000 \\ 3165000 & 1403000 & 0 & 620000 \\ 19120000 & 4601000 & 0 & 1804000 \\ 4030000 & 553000 & 0 & 313000 \\ 3879000 & 776000 & 0 & 628000 \\ 1460000 & 604000 & 0 & 293000 \\ 1611000 & 874000 & 0 & 605000 \\ 626000 & 476000 & 0 & 525000 \\ 1027000 & 451000 & 0 & 449000 \\ 965000 & 447000 & 0 & 224000 \\ 321000 & 309000 & 0 & 279000 \\ 1754000 & 504000 & 0 & 290000 \\ 5452000 & 1966000 & 755000 & 1059000 \\ 733000 & 188000 & 93000 & 132000 \\ 706000 & 205000 & 12000 & 251000 \\ 1285000 & 201000 & 101000 & 232000 \\ 197000 & 47000 & 3000 & 46000\end{array}$


Malayemys subtrijuga

Mauremys leprosa

Morenia ocellata

Pangshura tecta

Rhinoclemmys melanosterna

Siebenrockiella crassicollis
NHMUK 1920.1.20.2545

NHMUK unnumbered

NHMUK 87.3.11.7

NHMUK 1889.2.6.1

FMNH 44446

NHMUK 1864.9.2.47
Geoemydidae

Geoemydidae

Geoemydidae

Geoemydidae

Geoemydidae

Geoemydidae

$\begin{array}{cccc}784000 & 404000 & 0 & 413000 \\ 767000 & 291000 & 67000 & 180000 \\ 1452000 & 335000 & 109000 & 202000 \\ 2049000 & 409000 & 0 & 354000 \\ 440000 & 303000 & 0 & 180000 \\ 361000 & 206000 & 80000 & 128000\end{array}$




\section{Table 3 (on next page)}

Results of pGLS regressions of internal carotid artery size on stapedial artery size.

$\mathrm{CCl}$ stands for cross-sectional area of the internal carotid artery canal. CST stands for crosssectional area of stapedial artery canal. CTK-abbreviated model describes model only including chelonioids, trionychians, and kinosternoids. Remaining-abbreviated model includes all taxa not included in the CTK-model. Phylogenetic signal (lambda) was estimated during model fitting. $R^{2}$ is the generalized coefficient of determination described by Nagelkerke (1991). 


\begin{tabular}{llllll}
\hline Model & Lambda & Variable & Coefficient & P-Value & $\mathbf{R}^{2}$ \\
& & & & & \\
\hline$(\log 10(\mathrm{CCI}) \sim \log 10(\mathrm{CST}))_{\text {alltaxa }}$ & 0.957 & Intercept & 1.098 & $<0.001$ & 0.73 \\
& & Slope & 0.776 & $<0.001$ & \\
\hline$(\log 10(\mathrm{CCI}) \sim \log 10(\mathrm{CST}))_{\text {ctk }}$ & 0.738 & Intercept & 1.840 & $<0.001$ & 0.89 \\
& & Slope & 0.735 & $<0.001$ & \\
\hline$(\log 10(\mathrm{CCI}) \sim \log 10(\mathrm{CST}))_{\text {remaining }}$ & 0.400 & Intercept & 0.892 & 0.02 & 0.75 \\
& & Slope & 0.764 & $<0.001$ & \\
\hline
\end{tabular}

1 


\section{Table 4 (on next page)}

Comparative table summarizing the main differences between the internal carotid artery systems.

Abbreviations: bs, basisphenoid; CCB, canalis caroticus basisphenoidalis; $\mathbf{C C l}$, canalis caroticus internus; $\mathbf{C C L}$, canalis caroticus lateralis; $\mathbf{F P C C I}$, foramen posterius canalis carotici interni; int. car. art., internal carotid artery; pal. art., palatine artery; pro, prootic; pt, pterygoid; stap. art., stapedial artery; qu, quadrate. Note that NA (non-applicable) is used for the canalis caroticus lateralis either when the palatine artery is absent (pleurodires, Carettochelys insculpta, Platysternon megacephalum, testudinids, and some geoemydids), or because the palatine artery is not embedded in bone (chelonioids), and for the canalis caroticus basisphenoidalis as it is not fully ossified in Dermochelys coriacea. Columns of canals and foramina indicate bones involved in their formation. 


\begin{tabular}{|c|c|c|c|c|c|c|c|}
\hline Taxon & Specimen number & Clade & FPCCI & CCI & CCL & CCB & $\begin{array}{l}\text { Origin of the } \\
\text { mandibular artery }\end{array}$ \\
\hline Chelodina oblonga & NHMUK 64.12 .22 & Chelidae & $q u+$ pro & $\mathrm{qu}+$ pro + bs & NA & bs & stap. art. \\
\hline Chelus fimbriatus & NHMUK 81.9.27.4 & Chelidae & pro + bs & pro + bs & NA & bs & stap. art. \\
\hline Emydura subglobosa & PIMUZ lab 2009.37 & Chelidae & $\mathrm{qu}+$ pro $+\mathrm{bs}$ & $\mathrm{qu}+\mathrm{pro}+\mathrm{pt}+\mathrm{bs}$ & NA & bs & stap. art. \\
\hline Hydromedusa tectifera & SMF 70500 & Chelidae & pro & pro + bs & NA & bs & stap. art. \\
\hline Phrynops geoffroanus & SMF 45470 & Chelidae & $\mathrm{qu}+$ pro & $\mathrm{qu}+$ pro $+\mathrm{bs}$ & NA & bs & stap. art. \\
\hline Phrynops hilarii & NHMUK 91.3.16.1 & Chelidae & pro & pro + bs & NA & bs & stap. art. \\
\hline Podocnemis unifilis & NHMUK 60.4.16.9 & Podocnemididae & NA & NA & NA & bs & stap. art. \\
\hline Podocnemis unifilis & FMNH 45657 & Podocnemididae & NA & NA & NA & bs & stap. art. \\
\hline Pelomedusa subrufa & NMB 16229 & Pelomedusidae & pro + bs & pro + bs & NA & bs & stap. art. \\
\hline Pelusios subniger & NMB 16230 & Pelomedusidae & pro + bs & pro + bs & NA & bs & stap. art. \\
\hline Carettochelys insculpta & NHMUK 1903.7.10.1 & Carettochelyidae & $\mathrm{pt}$ & $\mathrm{pt}+\mathrm{bs}$ & NA & bs & stap. art. \\
\hline Carettochelys insculpta & SMF 56626 & Carettochelyidae & $\mathrm{pt}$ & $\mathrm{pt}+\mathrm{bs}$ & NA & bs & stap. art. \\
\hline Amyda cartilaginea & FMNH 244117 & Trionychidae & $\mathrm{pt}$ & pro $+\mathrm{pt}+\mathrm{bs}$ & $\mathrm{pt}+\mathrm{bs}$ & bs & int. car. art \\
\hline Apalone mutica & PCHP 2746 & Trionychidae & $\mathrm{pt}$ & pro $+\mathrm{pt}+\mathrm{bs}$ & $\mathrm{pt}+\mathrm{bs}$ & bs & int. car. art \\
\hline Apalone spinifera emoryi & FMNH 22178 & Trionychidae & $\mathrm{pt}$ & pro $+\mathrm{pt}+\mathrm{bs}$ & $\mathrm{pt}+\mathrm{bs}$ & bs & int. car. art \\
\hline Chitra indica & NHMUK 1926.12.16.1 & Trionychidae & $\mathrm{pt}$ & pro $+\mathrm{pt}+\mathrm{bs}$ & $\mathrm{pt}+\mathrm{bs}$ & bs & int. car. art \\
\hline Cyclanorbis senegalensis & NHMUK 65.5.9.21 & Trionychidae & $\mathrm{pt}$ & pro $+\mathrm{pt}+\mathrm{bs}$ & $\mathrm{pt}$ & bs & int. car. art \\
\hline Cycloderma frenatum & NHMUK 84.2.4.1 & Trionychidae & $\mathrm{pt}$ & $\mathrm{pt}+\mathrm{bs}$ & $\mathrm{pt}$ & bs & int. car. art \\
\hline Lissemys punctata & SMF 74141 & Trionychidae & $\mathrm{pt}$ & $\mathrm{pt}+\mathrm{bs}$ & $\mathrm{pt}$ & bs & int. car. art \\
\hline Pelodiscus sinensis & IW576-2b & Trionychidae & $\mathrm{pt}$ & pro $+p t+b s$ & $\mathrm{pt}+\mathrm{bs}$ & bs & int. car. art \\
\hline Kinosternon baurii & FMNH 211705 & Kinosternoidea & pro + pt & pro $+\mathrm{pt}+\mathrm{bs}$ & $\mathrm{pt}+\mathrm{bs}$ & bs & pal. art. \\
\hline Kinosternon scorpioides & SMF 71893 & Kinosternoidea & pro + pt & pro $+\mathrm{pt}+\mathrm{bs}$ & $\mathrm{pt}$ & bs & pal. art. \\
\hline Kinosternon subrubrum hippocrepis & FMNH 211711 & Kinosternoidea & pro + pt & pro $+\mathrm{pt}+\mathrm{bs}$ & pro $+\mathrm{pt}+\mathrm{bs}$ & bs & pal. art. \\
\hline Staurotypus salvinii & NHMUK 1879.1.7.5 & Kinosternoidea & pro + pt & pro $+\mathrm{pt}+\mathrm{bs}$ & pro $+\mathrm{pt}+\mathrm{bs}$ & bs & pal. art. \\
\hline Sternotherus minor & FMNH 211696 & Kinosternoidea & pro + pt & pro $+\mathrm{pt}+\mathrm{bs}$ & $\mathrm{pro}+\mathrm{pt}+\mathrm{bs}$ & bs & pal. art. \\
\hline
\end{tabular}


Dermatemys mawii

Chelydra serpentina

Macrochelys temminckii

\section{Caretta caretta}

Caretta caretta

Chelonia mydas

Eretmochelys imbricata

Lepidochelys olivacea

Natator depressus

\section{Dermochelys coriacea}

Dermochelys coriacea

Platysternon megacephalum

\section{Clemmys guttata}

Deirochelys reticularia

Emydoidea blandingii

Emys orbicularis

Glyptemys insculpta

Glyptemys muhlenbergii

Graptemys geographica

Pseudemys floridana

Terrapene coahuila

Terrapene ornata

\section{Agrionemys horsfieldii}

Aldabrachelys gigantea

Gopherus agassizii

Gopherus flavomarginatus

Gopherus polyphemus

\begin{tabular}{|c|c|c|}
\hline SMF 59463 & Kinosternoidea & pro + pt \\
\hline SMF 32846 & Chelydridae & pro + pt \\
\hline FMNH 22111 & Chelydridae & $\mathrm{pt}$ \\
\hline NHMUK 1938.1.9.1 & Cheloniidae & $\mathrm{pt}$ \\
\hline NHMUK 1940.3.15.1 & Cheloniidae & $\mathrm{pt}$ \\
\hline NHMUK 1969.776 & Cheloniidae & $\mathrm{pt}$ \\
\hline FMNH 22242 & Cheloniidae & $\mathrm{pt}$ \\
\hline SMNS 11070 & Cheloniidae & $\mathrm{pt}$ \\
\hline R112123 & Cheloniidae & $\mathrm{pt}$ \\
\hline FMNH 171756 & Dermochelyidae & pro + pt \\
\hline UMZC R3031 & Dermochelyidae & pro + pt \\
\hline SMF 69684 & Emysternia & $\mathrm{pt}$ \\
\hline FMNH 22114 & Emysternia & pro $+\mathrm{pt}+\mathrm{bs}$ \\
\hline FMNH 98754 & Emysternia & pro $+\mathrm{pt}+\mathrm{bs}$ \\
\hline FMNH 22144 & Emysternia & pro $+\mathrm{pt}+\mathrm{bs}$ \\
\hline SMF 1987 & Emysternia & pro + pt \\
\hline FMNH 22240 & Emysternia & pro $+\mathrm{pt}+\mathrm{bs}$ \\
\hline UF 85274 & Emysternia & pro + pt \\
\hline NHMUK 55.12.6.11 & Emysternia & pro + pt \\
\hline FMNH 8222 & Emysternia & $\mathrm{pt}+\mathrm{bs}$ \\
\hline FMNH 47372 & Emysternia & pro $+\mathrm{pt}$ \\
\hline FMNH 23014 & Emysternia & pro $+p t+b s$ \\
\hline PCHP 2929 & Testudinidae & pro + pt \\
\hline NHMUK 77.11.12.2 & Testudinidae & pro + pt \\
\hline FMNH 216746 & Testudinidae & pro + pt \\
\hline FMNH 98916 & Testudinidae & $\mathrm{pt}$ \\
\hline FMNH 211815 & Testudinidae & pro + pt \\
\hline
\end{tabular}

\section{pro $+p t+b s$}

pro $+\mathrm{pt}+\mathrm{bs}$

pro + pt + bs

$\mathrm{pt}$

$\mathrm{pt}$

$\mathrm{pt}+\mathrm{bs}$

$p t+b s$

$\mathrm{pt}$

$p t+b s$

pro $+p t+b s$
pro + pt + bs

pro + pt + bs

pro $+p t+b s$

pro + pt + bs

pro $+\mathrm{pt}+\mathrm{bs}$

pro $+p t+b s$

pro $+p t+b s$

pro $+\mathrm{pt}+\mathrm{bs}$

pro + pt + bs

$\mathrm{pt}+\mathrm{bs}$

pro + pt + bs

pro $+\mathrm{pt}+\mathrm{bs}$

pro $+\mathrm{pt}+\mathrm{bs}$

pro $+p t+b s$

pro $+p t+b s$

pro $+p t+b s$

pro $+p t+b s$ pro $+p t+b s \quad b s$

$\mathrm{pt}+\mathrm{bs}$

$\mathrm{pt}+\mathrm{bs}$

NA

NA

NA

NA

NA

NA

NA

NA

NA

$p t+b s$

$\mathrm{pt}+\mathrm{bs}$

$p t+b s$

$p t+b s$

$p t+b s$

$p t+b s$

$p t+b s$

$p t+b s$

$\mathrm{pt}+\mathrm{bs}$

$\mathrm{pt}+\mathrm{bs}$

NA

NA

NA

NA

NA stap./pal. art.

stap. art.

stap. art.

stap./pal. art. stap./pal. art. stap./pal. art. stap./pal. art. stap./pal. art. stap./pal. art.

unknown unknown

stap. art. stap. art. stap. art. stap. art. stap. art. stap. art. stap. art. stap. art. stap. art. stap. art. stap. art.

stap. art. stap. art. stap. art. stap. art. stap. art. 


\begin{tabular}{|c|c|c|c|c|c|c|c|}
\hline Indotestudo elongata & SMF 71585 & Testudinidae & pro + pt & pro $+p t+b s$ & NA & bs & stap. art. \\
\hline Indotestudo forstenii & SMF 73257 & Testudinidae & pro $+\mathrm{pt}$ & pro $+\mathrm{pt}+\mathrm{bs}$ & NA & bs & stap. art. \\
\hline Kinixys erosa & SMF 40166 & Testudinidae & pro $+\mathrm{pt}+\mathrm{bs}$ & pro $+\mathrm{pt}+\mathrm{bs}$ & NA & bs & stap. art. \\
\hline Malacochersus tornieri & SMF 58702 & Testudinidae & pro + pt & pro + pt + bs & NA & bs & stap. art. \\
\hline Psammobates tentorius verroxii & SMF 57142 & Testudinidae & $q u+$ pro $+p t+b s$ & $\mathrm{qu}+$ pro $+\mathrm{pt}+\mathrm{bs}$ & NA & bs & stap. art. \\
\hline Testudo marginata & FMNH 51672 & Testudinidae & pro + pt & pro $+\mathrm{pt}+\mathrm{bs}$ & NA & bs & stap. art. \\
\hline Batagur baska & NHMUK 67.9.28.7 & Geoemydidae & pro + pt & pro $+\mathrm{pt}+\mathrm{bs}$ & $\mathrm{pt}+\mathrm{bs}$ & bs & stap. art. \\
\hline Cuora amboinensis & NHMUK 69.42.145 & Geoemydidae & pro + pt & pro $+\mathrm{pt}+\mathrm{bs}$ & $\mathrm{pt}+\mathrm{bs}$ & bs & stap. art. \\
\hline Cyclemys dentata & NHMUK 97.11.22.3 & Geoemydidae & pro + pt & pro $+\mathrm{pt}+\mathrm{bs}$ & $\mathrm{pt}+\mathrm{bs}$ & bs & stap. art. \\
\hline Geoclemys hamiltonii & NHMUK 87.9.30.1 & Geoemydidae & $\mathrm{pt}$ & $\mathrm{pt}+\mathrm{bs}$ & $\mathrm{pt}+\mathrm{bs}$ & bs & stap. art. \\
\hline Geoemyda spengleri & FMNH 260381 & Geoemydidae & pro + pt & pro $+p t+b s$ & $\mathrm{pt}+\mathrm{bs}$ & bs & stap. art. \\
\hline Malayemys subtrijuga & NHMUK 1920.1.20.2545 & Geoemydidae & pro + pt & pro $+\mathrm{pt}+\mathrm{bs}$ & NA & bs & stap. art. \\
\hline Mauremys leprosa & NHMUK unnumbered & Geoemydidae & pro + pt & pro $+\mathrm{pt}+\mathrm{bs}$ & $\mathrm{pt}+\mathrm{bs}$ & bs & stap. art. \\
\hline Morenia ocellata & NHMUK 87.3.11.7 & Geoemydidae & pro + pt & pro $+\mathrm{pt}+\mathrm{bs}$ & $\mathrm{pt}+\mathrm{bs}$ & bs & stap. art. \\
\hline Pangshura tecta & NHMUK 1889.2.6.1 & Geoemydidae & pro + pt & pro $+\mathrm{pt}+\mathrm{bs}$ & NA & bs & stap. art. \\
\hline Rhinoclemmys melanosterna & FMNH 44446 & Geoemydidae & pro + pt & pro $+\mathrm{pt}+\mathrm{bs}$ & NA & bs & stap. art. \\
\hline Siebenrockiella crassicollis & NHMUK 1864.9.2.47 & Geoemydidae & pro + pt & pro $+\mathrm{pt}+\mathrm{bs}$ & $\mathrm{pt}$ & $\mathrm{pt}+\mathrm{bs}$ & stap. art. \\
\hline
\end{tabular}




\section{Table 5 (on next page)}

Comparative table summarizing the main differences between the facial nerve systems.

Abbreviations: bs, basisphenoid; cci, canalis caroticus internus; ccl, canalis caroticus lateralis; ccv, canalis cavernosus; cnhd, canalis nervus hyomandibularis distalis; cnhp, canalis nervus hyomandibularis proximalis; cnv, canalis nervus vidianus; conf, hyomandibular nerve confluent with the canalis cavernosus; epi, epipterygoid; pal, palatine; par, parietal; pro, prootic; pt, pterygoid; scc, short-cut canal; scv, sulcus cavernosus; sulcus, hyomandibular nerve contained in a sulcus within the canalis cavernosus. Note that NA (non-applicable) is used when the canalis nervus vidianus is absent (chelonioids). 


\section{Taxon}

Specimen number Clade

Geniculate Hyomandibular

ganglion nerve path

position

Chelodina oblonga
Chelus fimbriatus
Emydura subglobosa
Hydromedusa tectifera
Phrynops geoffroanus
Phrynops hilarii

Podocnemis unifilis

Podocnemis unifilis

Pelomedusa subrufa

Pelusios subniger

Carettochelys insculpta

Carettochelys insculpta

\section{Amyda cartilaginea}

Apalone mutica

Apalone spinifera emoryi

Chitra indica

Cyclanorbis senegalensis

Cycloderma frenatum

Lissemys punctata

Pelodiscus sinensis

\section{Kinosternon baurii}

Kinosternon scorpioides

Kinosternon subrubrum hippocrepis
NHMUK 64.12.22

NHMUK 81.9.27.4

PIMUZ lab 2009.37

SMF 70500

SMF 45470

NHMUK 91.3.16.1

NHMUK 60.4.16.9

NMB 16229

NMB 16230

NHMUK 1903.7.10.1

SMF 56626

FMNH 244117

PCHP 2746

FMNH 22178

NHMUK 1926.12.16.1

NHMUK 65.5.9.21

NHMUK 84.2.4.1

SMF 74141

IW576-2b

FMNH 211705

SMF 71893

FMNH 211711
FMNH 45657
Chelidae

Chelidae

Chelidae

Chelidae

Chelidae

Chelidae

Podocnemididae pro

Podocnemididae pro

Pelomedusidae cci

Pelomedusidae cci

Carettochelyidae pro

Carettochelyidae pro

Trionychidae ccv

Trionychidae

Trionychidae

Trionychidae

Trionychidae

Trionychidae

Trionychidae

Trionychidae

Kinosternoidea

Kinosternoidea

Kinosternoidea cnhp + sulcus

cnhp + sulcus

cnhp

cnhp + sulcus

cnhp + sulcus

cnhp + sulcus

cnhp + sulcus +

cnhd

cnhp + sulcus +

cnhd

cnhp + sulcus

cnhp + sulcus

cnhp + sulcus

cnhp + sulcus

conf

sulcus

sulcus

cnhp

cnhp

cnhp

cnhp

sulcus

sulcus

sulcus

sulcus
Vidian nerve path

$\mathrm{cci}+\mathrm{cnv}$

cnv

$\mathrm{cci}+\mathrm{cnv}$

$\mathrm{cci}+\mathrm{cnv}$

$\mathrm{cci}+\mathrm{cnv}$

$\mathrm{cci}+\mathrm{cnv}$

$\mathrm{scv}+\mathrm{cnv}$

cnv

$\operatorname{cci}+\mathrm{cnv}$

cnv

$\mathrm{cci}+\mathrm{cnv}$

cci + cnv

$\mathrm{cci}+\mathrm{ccl}+\mathrm{scV}+\mathrm{cnv}$

$\mathrm{cci}+\mathrm{ccl}+\mathrm{scV}+\mathrm{cnv}$

$\mathrm{cci}+\mathrm{ccl}+\mathrm{scV}+\mathrm{cnv}$

$\mathrm{cci}+\mathrm{ccl}+\mathrm{scv}+\mathrm{cnv}$

$\mathrm{cci}+\mathrm{ccl}+\mathrm{scV}+\mathrm{cnv}$

$\mathrm{cci}+\mathrm{scv}+\mathrm{cnv}$

$\mathrm{cci}+\mathrm{ccl}+\mathrm{scv}+\mathrm{cnv}$

$\mathrm{cci}+\mathrm{ccl}+\mathrm{scv}+\mathrm{cnv}$

$\mathrm{cci}+\mathrm{ccl}+\mathrm{scV}+\mathrm{cnv}$

$\mathrm{cci}+\mathrm{ccl}+\mathrm{scV}+\mathrm{cnv}$

$\mathrm{cci}+\mathrm{ccl}+\mathrm{scV}+\mathrm{cnv}$
Bones forming the canalis nervus vidianus

pro + pt + bs

pro + pt + par

pro + pt

pro + pt + bs

pro $+\mathrm{pt}$

pro + pt

$\mathrm{pt}+\mathrm{pal}+\mathrm{par}$

$\mathrm{pt}+\mathrm{pal}+\mathrm{par}$

pro $+p t+b s$

pt + pal

$p t+p a l$

pt + pal

pt + pal

pal

pal

pal

$\mathrm{pt}+\mathrm{pal}+\mathrm{par}$

$p t+$ pal

pal

pt + pal + par
pt + epi + pal
pt + pal 
Staurotypus salvinii

Sternotherus minor

Dermatemys mawii

Chelydra serpentina

Macrochelys temminckii

Caretta caretta

Caretta caretta

Chelonia mydas

Eretmochelys imbricata

Lepidochelys olivacea

Natator depressus

Dermochelys coriacea

Dermochelys coriacea

Platysternon megacephalum

Clemmys guttata

Deirochelys reticularia

Emydoidea blandingii

Emys orbicularis

Glyptemys insculpta

Glyptemys muhlenbergii

Graptemys geographica

Pseudemys floridana

Terrapene coahuila

Terrapene ornata

Agrionemys horsfieldii

Aldabrachelys gigantea

Gopherus agassizii

NHMUK 1879.1.7.5
FMNH 211696
SMF 59463
SMF 32846
FMNH 22111
NHMUK 1938.1.9.1
NHMUK 1940.3.15.1
NHMUK 1969.776
FMNH 22242
SMNS 11070
R112123

Kinosternoidea

Kinosternoidea

Kinosternoidea

$\mathrm{ccV}$

Chelydridae

Chelydridae

$\mathrm{ccV}$

$\mathrm{ccV}$

Cheloniidae

Cheloniidae

Cheloniidae

Cheloniidae

Cheloniidae

Cheloniidae

\section{FMNH 171756 \\ UMZC R3031}

\section{SMF 69684 \\ FMNH 22114 \\ FMNH 98754 \\ FMNH 22144 \\ SMF 1987 \\ FMNH 22240 \\ UF 85274 \\ NHMUK 55.12.6.11 \\ FMNH 8222 \\ FMNH 47372 \\ FMNH 23014}

PCHP 2929

NHMUK 77.11.12.2

FMNH 216746
Dermochelyidae cci

Dermochelyidae cci

Emysternia

Emysternia

Emysternia

Emysternia

Emysternia

Emysternia

Emysternia

Emysternia

Emysternia

Emysternia

Emysternia

Testudinidae

Testudinidae

Testudinidae conf

sulcus

conf

sulcus

conf

conf

conf

sulcus

conf

sulcus

conf

cnhp + conf

cnhp + conf

conf

sulcus

sulcus

sulcus

sulcus

conf

conf

sulcus

conf

sulcus

sulcus

sulcus

cnhd

sulcus cci + cnv

$\mathrm{cci}+\mathrm{ccl}+\mathrm{scV}+\mathrm{cnv}$

$\mathrm{cci}+\mathrm{ccl}+\mathrm{scv}+\mathrm{cnv}$

cci + cnv

cci + cnv

$\mathrm{cci}+\mathrm{scv}$

$\mathrm{cci}+\mathrm{scv}$

$\mathrm{cci}+\mathrm{scv}$

$\mathrm{cci}+\mathrm{scv}$

$\mathrm{cci}+\mathrm{scv}$

$\mathrm{cci}+\mathrm{scv}$

$\mathrm{cci}+\mathrm{scv}$

$\mathrm{cci}+\mathrm{scv}$

$\mathrm{cci}+\mathrm{cnv}$

$\mathrm{cci}+\mathrm{ccl}+\mathrm{scv}+\mathrm{cnv}$

$\mathrm{cci}+\mathrm{ccl}+\mathrm{cnv}$

$\mathrm{cci}+\mathrm{ccl}+\mathrm{scv}+\mathrm{cnv}$

$\mathrm{cci}+\mathrm{ccl}+\mathrm{scv}+\mathrm{cnv}$

$\mathrm{cci}+\mathrm{scc}+\mathrm{ccl}+\mathrm{cnv}$

$\mathrm{ccl}+\mathrm{scv}+\mathrm{cnv}$

$\mathrm{cci}+\mathrm{ccl}+\mathrm{cnv}$

$\mathrm{cci}+\mathrm{ccl}+\mathrm{cnv}$

$\mathrm{cci}+\mathrm{ccl}+\mathrm{cnv}$

$\mathrm{cci}+\mathrm{ccl}+\mathrm{cnv}$

cci + cnv

cci + cnv

cci + cnv $\mathrm{pt}+\mathrm{pal}$

$\mathrm{pt}+\mathrm{pal}$

pal

$\mathrm{pt}$

pt

NA

NA

NA

NA

NA

NA

NA

NA

$\mathrm{pt}+\mathrm{pal}$

$\mathrm{pt}+$ epi

pro + pt + bs + epi + par

$\mathrm{pt}+$ epi + par

pt + epi + par

pt

pt

$\mathrm{pt}+\mathrm{pal}$

$\mathrm{pt}+\mathrm{pal}$

$\mathrm{pt}+$ epi

pt + epi

pro + pt + pal

$\mathrm{pt}+$ epi + pal

$\mathrm{pt}+\mathrm{pal}$ 
Gopherus flavomarginatus

Gopherus polyphemus

Indotestudo elongata

Indotestudo forstenii

Kinixys erosa

Malacochersus tornieri

Psammobates tentorius verroxii

Testudo marginata

\section{Batagur baska}

Cuora amboinensis

Cyclemys dentata

Geoclemys hamiltonii

Geoemyda spengleri

Malayemys subtrijuga

Mauremys leprosa

Morenia ocellata

Pangshura tecta

Rhinoclemmys melanosterna

Siebenrockiella crassicollis

2

$\begin{array}{llll}\text { FMNH 98916 } & \text { Testudinidae } & \text { ccv } & \text { conf } \\ \text { FMNH 211815 } & \text { Testudinidae } & \text { ccv } & \text { cnhd } \\ \text { SMF 71585 } & \text { Testudinidae } & \text { pro } & \text { cnhp + conf } \\ \text { SMF 73257 } & \text { Testudinidae } & \text { pro } & \text { sulcus } \\ \text { SMF 40166 } & \text { Testudinidae } & \text { ccv } & \text { sulcus } \\ \text { SMF 58702 } & \text { Testudinidae } & \text { ccv } & \text { sulcus } \\ \text { SMF 57142 } & \text { Testudinidae } & \text { pro/cci } & \text { sulcus } \\ \text { FMNH 51672 } & \text { Testudinidae } & \text { ccv } & \text { sulcus } \\ & & & \\ \text { NHMUK 67.9.28.7 } & \text { Geoemydidae } & \text { ccv } & \text { sulcus } \\ \text { NHMUK 69.42.145 } & \text { Geoemydidae } & \text { ccv } & \text { sulcus } \\ \text { NHMUK 97.11.22.3 } & \text { Geoemydidae } & \text { ccv } & \text { sulcus } \\ \text { NHMUK 87.9.30.1 } & \text { Geoemydidae } & \text { ccv } & \text { sulcus } \\ \text { FMNH 260381 } & \text { Geoemydidae } & \text { ccv } & \text { sulcus } \\ \text { NHMUK 1920.1.20.2545 } & \text { Geoemydidae } & \text { ccv } & \text { sulcus } \\ \text { NHMUK unnumbered } & \text { Geoemydidae } & \text { pro } & \text { sulcus } \\ \text { NHMUK 87.3.11.7 } & \text { Geoemydidae } & \text { ccv } & \text { conf } \\ \text { NHMUK 1889.2.6.1 } & \text { Geoemydidae } & \text { ccv } & \text { sulcus } \\ \text { FMNH 44446 } & \text { Geoemydidae } & \text { cci } & \text { sulcus } \\ \text { NHMUK 1864.9.2.47 } & \text { Geoemydidae } & \text { ccv } & \text { sulcus }\end{array}$

cci + cnv

cci + cnv

cci + cnv

cci + cnv

cnv

cci + cnv

cci + cnv

cnv
$\mathrm{cci}+\mathrm{ccl}+\mathrm{scv}+\mathrm{cnv}$
$\mathrm{cci}+\mathrm{ccl}+\mathrm{scv}+\mathrm{cnv}$
cnv
$\mathrm{cci}+\mathrm{ccl}+\mathrm{scv}+\mathrm{cnv}$
$\mathrm{ccl}+\mathrm{cnv}$
cci + cnv
$\mathrm{cci}+\mathrm{scc}+\mathrm{ccl}+\mathrm{scv}+\mathrm{cnv}$
$\mathrm{cci}+\mathrm{scc}+\mathrm{ccl}+\mathrm{scv}+\mathrm{cnv}$
cci + cnv
cci + cnv
$\mathrm{cci}+\mathrm{ccl}+\mathrm{scv}+\mathrm{cnv}$

$\mathrm{pt}+$ epi + pal + par

pro $+\mathrm{pt}+\mathrm{pal}$

$\mathrm{pt}+\mathrm{pal}$

$\mathrm{pt}$

pro + pt + pal + par

pro + pt + pal

$\mathrm{pt}+\mathrm{pal}$

pro + pt + epi + pal + par

$\mathrm{pt}+\mathrm{pal}$

$\mathrm{pt}+\mathrm{pal}+\mathrm{par}$

pro + pt + par

$\mathrm{pt}+\mathrm{pal}+\mathrm{par}$

pro + pt

$\mathrm{pt}+\mathrm{pal}+\mathrm{par}$

$\mathrm{pt}+$ epi + par

$\mathrm{pt}+\mathrm{pal}+\mathrm{par}$

$\mathrm{pt}+\mathrm{pal}$

$\mathrm{pt}+$ epi $+\mathrm{pal}+$ par

$\mathrm{pt}+\mathrm{pal}+\mathrm{par}$ 